\title{
Pressurized Metered-Dose (pMDI) Aerosol Spray Deposition in Large- and Medium-Volume Spacers
}

by

Nicholas Ogrodnik, B. Eng.

A thesis submitted to the Faculty of Graduate and Postdoctoral Affairs in partial fulfillment of the requirements for the degree of

Master of Applied Science

in

Mechanical Engineering

Carleton University

Ottawa, Ontario

(C) Copyright

Nicholas Ogrodnik, 2020 


\section{Abstract}

Spacers (or holding chambers) are typically used with pressurized metered-dose inhalers (pMDIs) by patients suffering from chronic lower respiratory tract diseases such as asthma and chronic obstructive pulmonary disease (COPD). Attachments such as spacers provide a number of benefits including: a reduction of the "Cold Freon effect ${ }^{1 "}$, better coordination (between pMDI actuation and inhalation), and a reduction of the inertial impaction of the medication in the oropharyngeal tract and oropharynx. These factors improve the inhalation of medication resulting in a larger percentage of medication delivered to the lungs when using a spacer device. However, spacer devices can also cause a loss of medication delivered by the pMDI due to deposition of the medication on the walls of the spacer itself. As such, it is important to understand the mechanisms which cause this medication loss to allow for future spacer devices to be designed more efficiently.

Regional deposition of the medication, salbutamol sulphate, was studied in a medium- and large- volume spacer, namely, the Volumatic ${ }^{\mathrm{TM}}$ and OptiChamber ${ }^{\circledR}$ spacer. $^{\circ}$ This study was completed using both experimental and numerical analyses. Experiments were conducted at typical inspiratory flow rates ranging from 30 to $60 \mathrm{~L} / \mathrm{min}$, which were typical for medication delivery. The amount of deposition of medication in the spacer device was assessed using spectrophotometry. Computational fluid dynamics (CFD) was also used to allow the implementation of particle tracking and quantify the

\footnotetext{
${ }^{1}$ It should be noted that chlorofluorocarbons (CFCs) are no longer used in pMDIs and, as such, Freon is no longer present in the inhalers. However, the concept of a cold plume impacting the back of the throat (which was described by the Cold Freon effect) is still present in pMDIs even with current propellants.
} 
deposition (and its mechanism) numerically at a flow rate of $30 \mathrm{~L} / \mathrm{min}$. Simulations used both mean flow and turbulent particle tracking, applying unsteady Reynolds-averaged Navier-Stokes (URANS) equations with a shear stress transport (SST) turbulence model. Deposition of salbutamol sulphate in the Volumatic ${ }^{\mathrm{TM}}$ and OptiChamber ${ }^{\circledR}$ spacers was found to be greater in the lower half as opposed to the upper half of the spacer due to a downward spray angle. Additionally, it was determined more deposition of medication could be expected in the distal half of the spacer as opposed to the proximal half. An increase in flow rate demonstrated a minimal increase in the medication delivered to the inline filter which was analogous to that reaching the patient. The numerical analysis demonstrated that turbulence effects are likely to cause deposition in both the Volumatic $^{\mathrm{TM}}$ and OptiChamber ${ }^{\circledR}$ spacer.

Results suggested that a larger flow rate does not necessarily allow for more medication to be delivered, it acts only to shift the region of the deposition. As such, it is conjectured that each spacer should have an optimal flow rate as to where the most medication will be delivered without excessive inhalation by the patient. 


\section{Acknowledgements}

I would like to express my sincerest gratitude to my thesis supervisor, Professor Edgar Matida, for all the support, guidance and encouragement provided throughout my studies, during both my undergraduate and graduate research. I always appreciated your patient approach and positive attitude throughout my academic journey. This gratitude is extended to my colleagues as well, providing me clarity in times of stress, always willing to lend a helping hand and to listen whenever I needed a voice of reason.

At home, I would like to thank my partner, as without her encouragement, and her never-ending love and support, I would not be where I am today. Your grace and passion mean the world to me. To my parents and brother, as well, who supported my education, encouraged me to be my best and were always there with advice and a home cooked meal whenever I needed to vent. I am sure, Mom, you would be happy to hear I am finally done! To my partner's family, who were always so understanding and caring. You are a wonderful bunch, bringing so much positivity and kindness (and great food!) to my life. Lastly, to my two cats, who sat with me throughout my edits - and who also found enjoyment from walking across my keyboard. I would like to dedicate any typos found in my thesis to them especially.

\footnotetext{
"All we have to decide is what to do with the time that is given us" - J.R.R. Tolkien
} 


\section{Table of Contents}

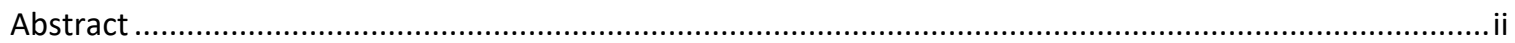

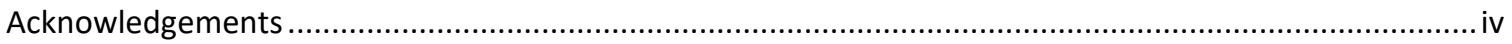

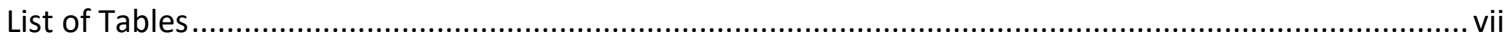

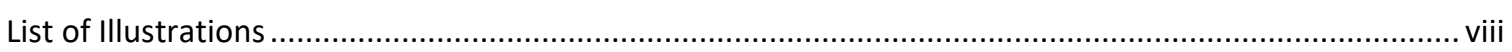

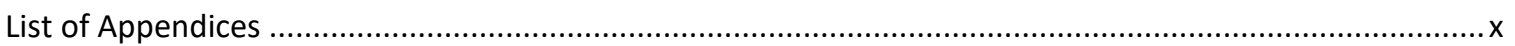

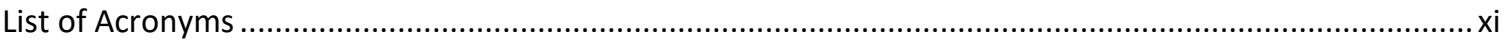

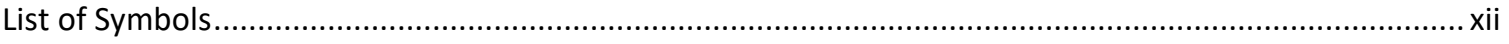

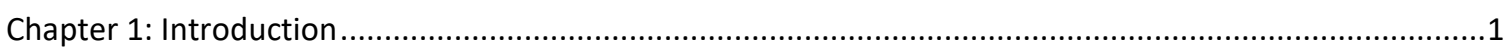

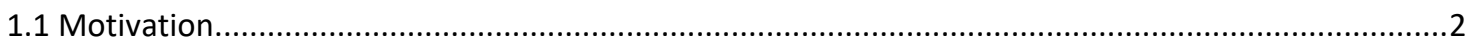

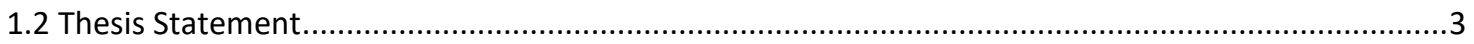

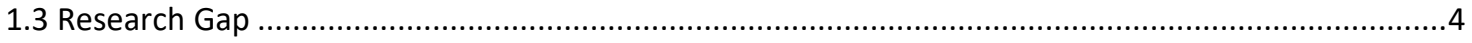

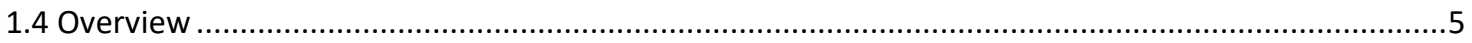

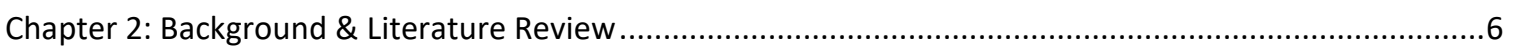

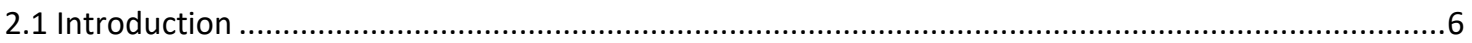

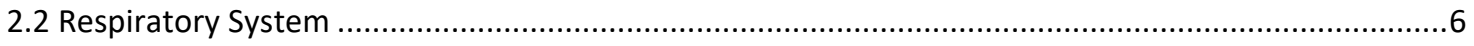

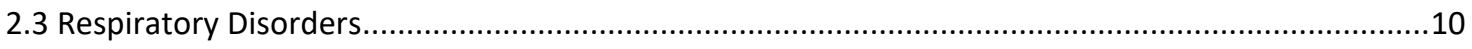

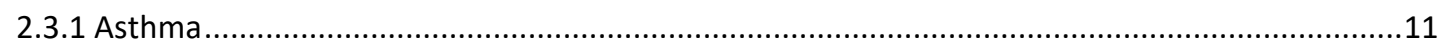

2.3.2 Chronic Obstructive Pulmonary Disease .................................................................. 15

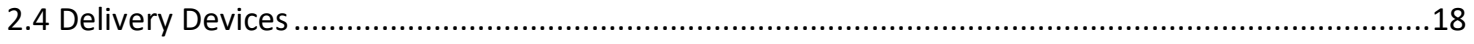

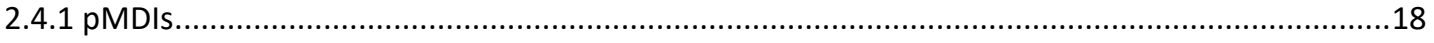

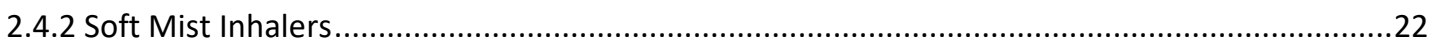

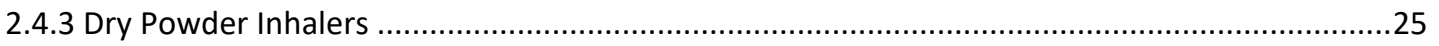

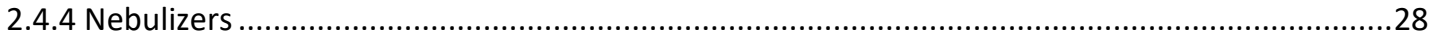

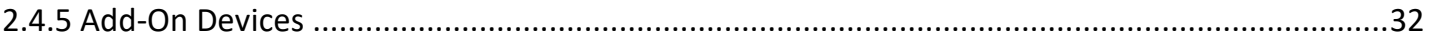

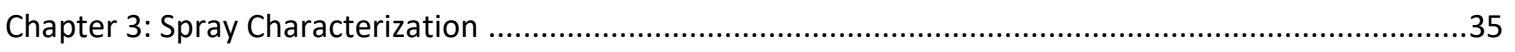

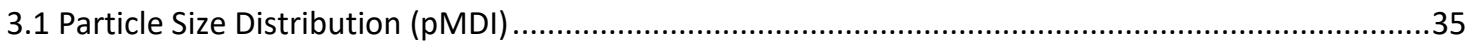

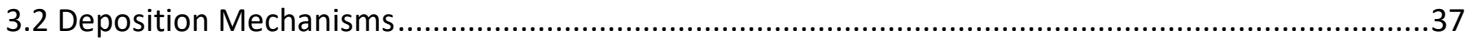

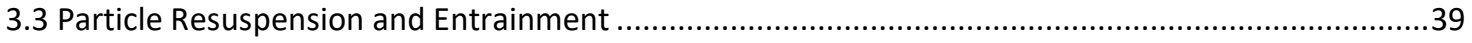

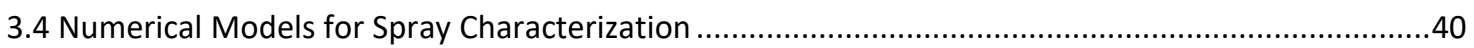

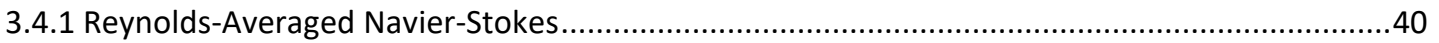

3.4.2 Unsteady Reynolds-Averaged Navier-Stokes ...............................................................42

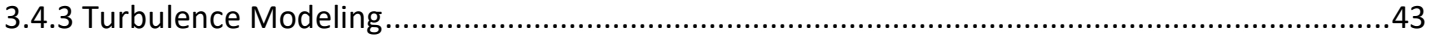

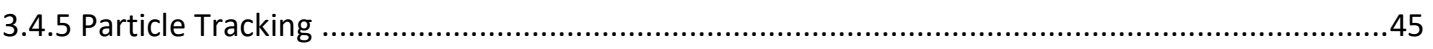

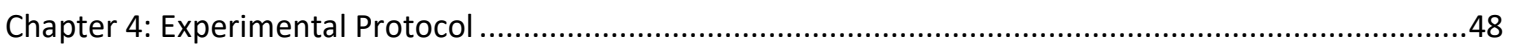

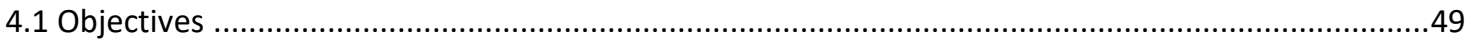

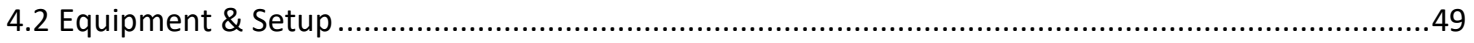




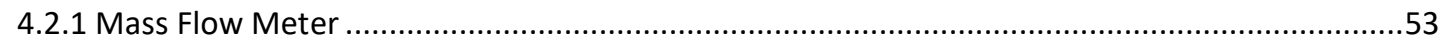

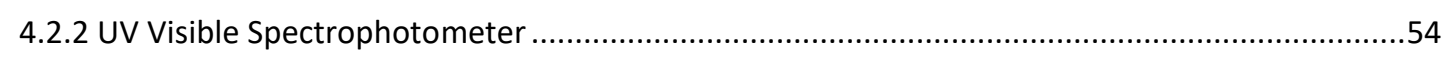

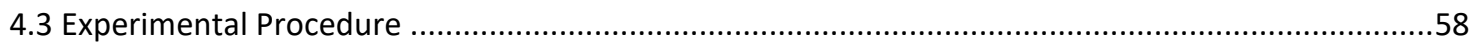

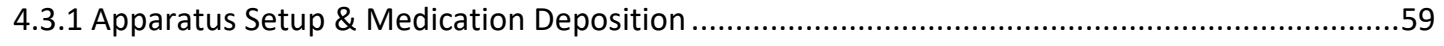

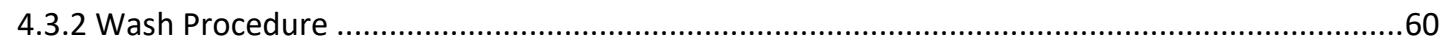

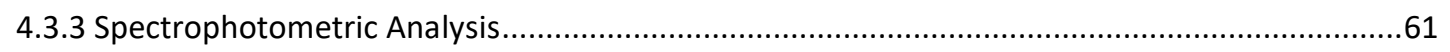

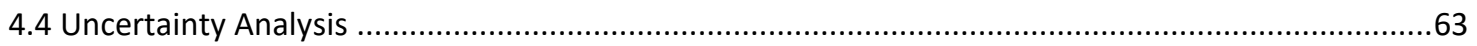

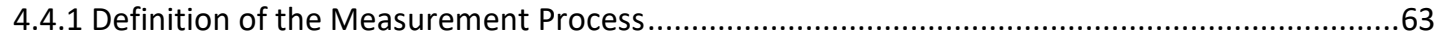

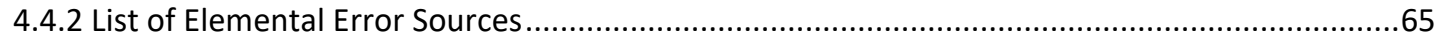

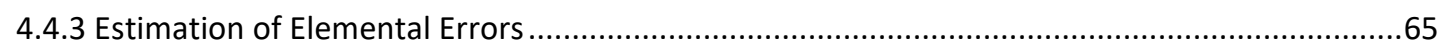

4.4.4 Propagation of Bias and Random Uncertainties ..............................................................67

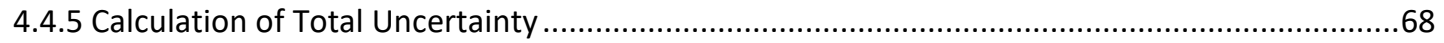

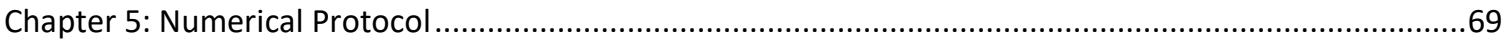

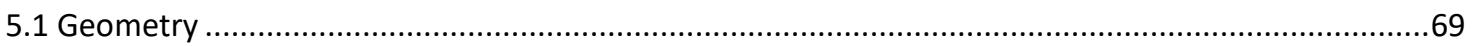

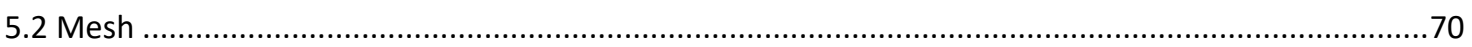

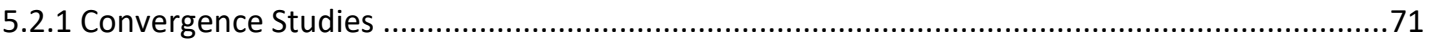

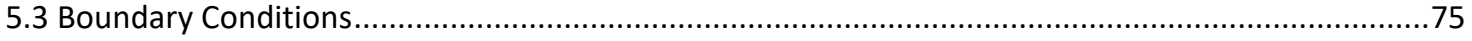

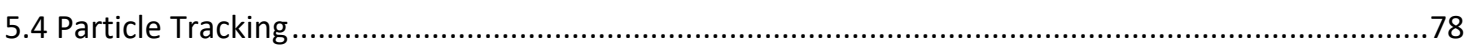

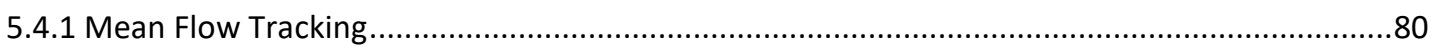

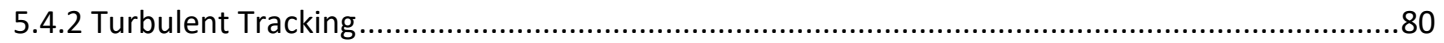

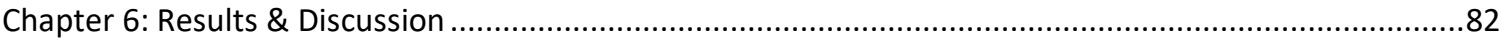

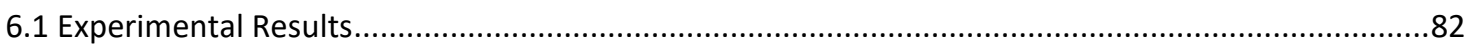

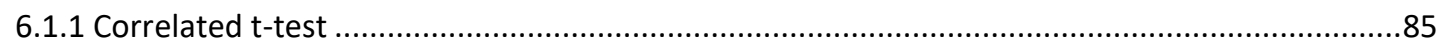

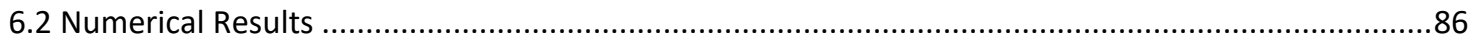

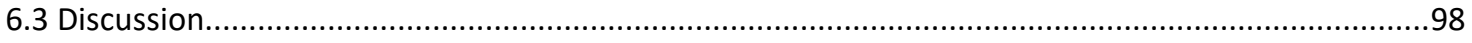

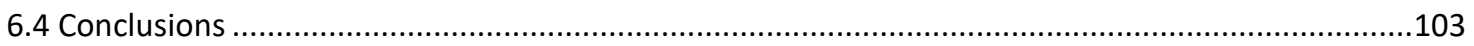

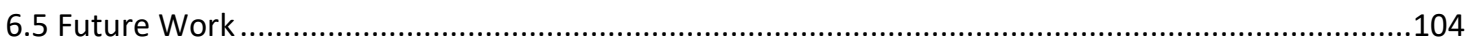

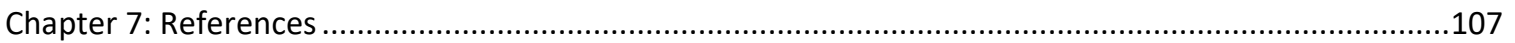

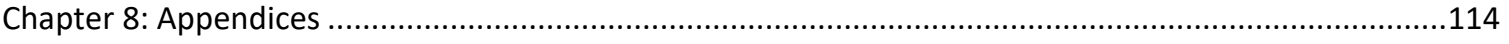

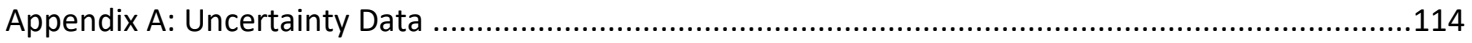

Appendix B: Supplementary Background Information ...............................................................115

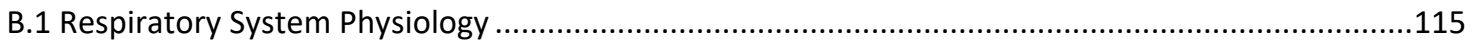

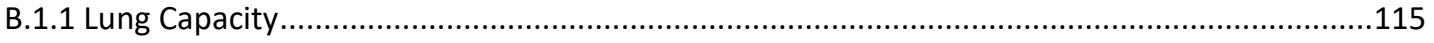

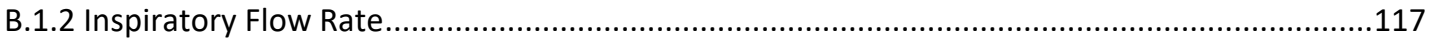

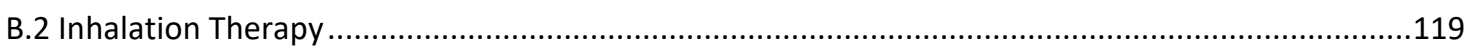

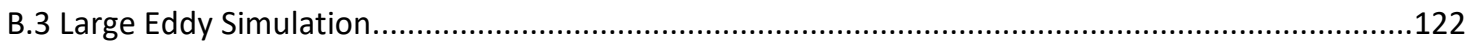




\section{List of Tables}

Table 2.1: Characteristics of each stage of COPD (Adapted from [30]) .................................... 16

Table 2.2: GOLD COPD management plan details (Adapted from [30]) ..................................... 18

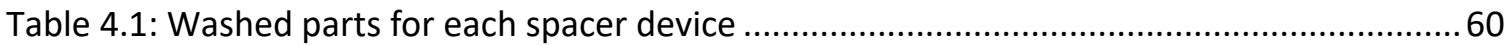

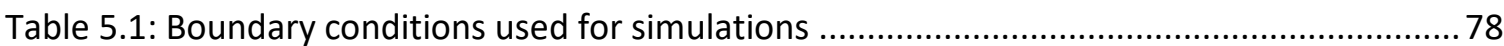

Table 5.2: Fluid domain settings used for simulations .................................................................... 78

Table 6.1: Calculated t values and two-tailed probability to prove statistical difference between the experimental deposition found on the lower versus upper regions and distal versus proximal regions of the Volumatic ${ }^{\mathrm{TM}}$ spacer for each corresponding flow rate

Table 6.2: Calculated t values and two-tailed probability to prove statistical difference between the experimental deposition found on the lower versus upper regions and distal versus proximal regions of the OptiChamber ${ }^{\circledR}$ spacer for each corresponding flow rate.......................................86

Table 6.3: Hypothetical dose delivery with OptiChamber ${ }^{\oplus}$ at $30 \mathrm{~L} / \mathrm{min}$ versus $60 \mathrm{~L} / \mathrm{min} . . . . . . . . .102$

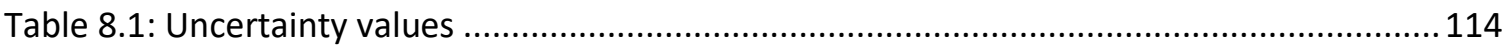

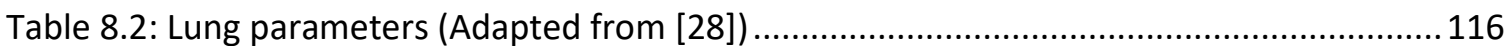




\section{List of Illustrations}

Figure 1.1: Medication delivery to patient via pMDI (Adapted from [6]) ................................... 2

Figure 2.1: The respiratory system (Adapted from [17]) …....................................................

Figure 2.2: Schematic representation of the bronchial tree. Generations 1 to 16 are the conductive airways. Generations 17 to 24 are the respiratory zone (Adapted from [19]) ............8

Figure 2.3: Diffusion of oxygen into the blood stream (Adapted from [17]) ............................... 9

Figure 2.4: Illustration of normal airways versus those from an asthmatic (Adapted from [26]).12

Figure 2.5: Lung capacities typically measured by a spirometer (Adapted from [28]).................14

Figure 2.6: Example flow volume plot from spirometer measurements..................................... 14

Figure 2.7: Illustration of pMDI components (Adapted from [35]) …......................................19

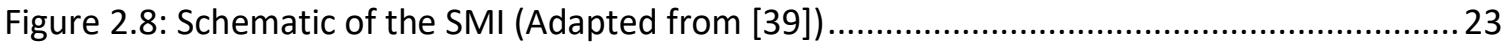

Figure 2.9: Schematic of the SMI Uniblock nozzle (Adapted from [40]).....................................24

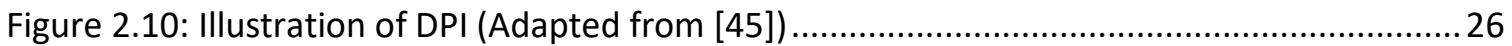

Figure 2.11: Illustration of the fluidization of a powder bed in a DPI (Adapted from [46]) ..........27

Figure 2.12: Schematic of jet nebulisation (Adapted from [48]) ................................................30

Figure 2.13: Illustration of an ultrasonic nebulizer (Adapted from [48]) ....................................31

Figure 2.14: Spacer devices typically used with a pMDI (from left to right; Aerochamber ${ }^{\circledast}$,

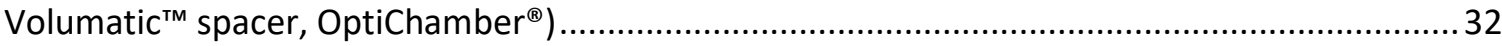

Figure 3.1: Deposition regions based on particle size (Adapted from [52]) ................................36

Figure 4.1: Illustration of $750 \mathrm{~mL}$ large-volume Volumatic ${ }^{\mathrm{TM}}$ spacer ..........................................48

Figure 4.2: Schematic of $218 \mathrm{~mL}$ medium-volume OptiChamber ${ }^{\circledR}$ Spacer. (A) OptiChamber ${ }^{\circledR}$ with mouthpiece attached. (B) OptiChamber ${ }^{\circledR}$ with mouthpiece removed, exposing the valved portion of the spacer

Figure 4.3: Schematic of Volumatic ${ }^{\mathrm{TM}}$ spacer divided into quarters: distal lower, distal upper, proximal lower, and proximal upper. An adaptor was used to attach the filter to the prototyped Volumatic ${ }^{\mathrm{TM}}$ spacer model

Figure 4.4: Schematic of OptiChamber ${ }^{\circledast}$ spacer divided into quarters: distal lower, distal upper, proximal lower, and proximal upper. A mouthpiece and adaptor were used to attach the filter and $\mathrm{pMDI}$ to the prototyped OptiChamber ${ }^{\circledR}$ spacer model

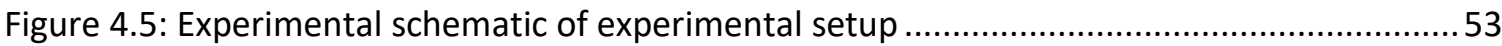

Figure 4.6: Schematic of scanning spectrophotometer (Adapted from [62]) ...............................58

Figure 4.7: Salbutamol sulphate calibration curve for spectrophotometric analysis ...................62

Figure 5.1: 3D render of the Volumatic ${ }^{T M}$ spacer and pMDI using ICEM by ANSYS ......................70

Figure 5.2: 3D render of the OptiChamber ${ }^{\circledR}$ spacer and pMDI using ICEM by ANSYS ..................70 
Figure 5.3: Unstructured mesh of Volumatic ${ }^{T M}$ spacer geometry ( 6 million elements with prism layers)

Figure 5.4: Unstructured mesh of OptiChamber ${ }^{\circledast}$ spacer geometry (6 million elements with prism

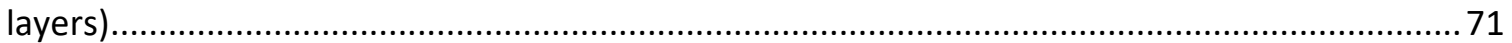

Figure 5.5: Velocity along centreline for Volumatic ${ }^{\mathrm{TM}}$ spacer .................................................. 72

Figure 5.6: Velocity along centreline for OptiChamber ${ }^{\circledR}$ spacer................................................. 73

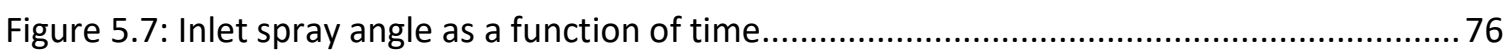

Figure 5.8: Implemented boundary conditions for the Volumatic ${ }^{\mathrm{TM}}$ spacer ................................77

Figure 5.9: Implemented boundary conditions for the OptiChamber ${ }^{\circledR}$ spacer ............................77

Figure 6.1: Experimental results of the percent deposition of medication in the upper and lower

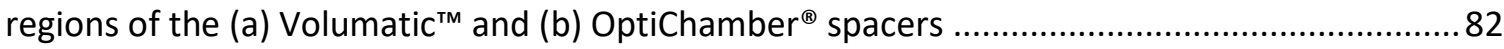

Figure 6.2: Experimental results of the percent deposition of medication in the spacer and filter of the (a) Volumatic ${ }^{\mathrm{TM}}$ and (b) OptiChamber ${ }^{\circledR}$ spacers

Figure 6.3: Experimental results of the percent deposition of medication in the proximal and distal of the (a) Volumatic ${ }^{\mathrm{TM}}$ and (b) OptiChamber ${ }^{\circledR}$ spacers.

Figure 6.4: Mean flow (A) and turbulent (B) particle tracks for the Volumatic ${ }^{\mathrm{TM}}$ (top) and OptiChamber $^{\circledR}$ (bottom) spacer.

Figure 6.5: Numerical results of the percent deposition of medication in the upper and lower regions of the (a) Volumatic ${ }^{\mathrm{TM}}$ and (b) OptiChamber ${ }^{\circledast}$ spacers using mean-flow tracking... 89

Figure 6.6: Numerical results of the percent deposition of medication in the proximal and distal regions of the (a) Volumatic ${ }^{\mathrm{TM}}$ and (b) OptiChamber ${ }^{\circledR}$ spacers using mean-flow tracking... 89

Figure 6.7: Numerical results of the percent deposition of medication in the upper and lower regions of the (a) Volumatic ${ }^{\mathrm{TM}}$ and (b) OptiChamber ${ }^{\circledR}$ spacers using turbulent tracking.....

Figure 6.8: Numerical results of the percent deposition of medication in the proximal and distal regions of the (a) Volumatic ${ }^{\mathrm{TM}}$ and (b) OptiChamber ${ }^{\circledR}$ spacers using turbulent tracking..... 91

Figure 6.9: Numerical results of the turbulence kinetic energy in the Volumatic ${ }^{\mathrm{TM}}$ spacer at 30 $\mathrm{L} / \mathrm{min}$.

Figure 6.10: Numerical results of the turbulence kinetic energy in the OptiChamber ${ }^{\circledR}$ spacer at $30 \mathrm{~L} / \mathrm{min}$

Figure 6.11: Numerical results of the velocity in the Volumatic $^{\mathrm{TM}}$ spacer at $30 \mathrm{~L} / \mathrm{min} . . \ldots \ldots \ldots \ldots . . . . . .97$

Figure 6.12: Numerical results of the velocity in the OptiChamber ${ }^{\circledast}$ spacer at $30 \mathrm{~L} / \mathrm{min}$.............98

Figure 8.1: Illustration of filtered velocity and instantaneous velocity (Adapted from [83]) ......122 


\section{List of Appendices}

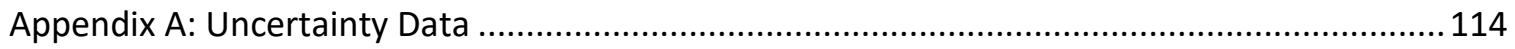

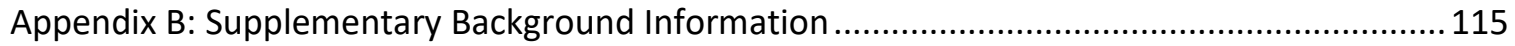




\section{List of Acronyms}

\begin{tabular}{|c|c|}
\hline Acronym & Definition \\
\hline $\mathrm{CFC}$ & Chlorofluorocarbon \\
\hline CFC-11 & CFC Trichlorofluoromethane \\
\hline CFC-12 & CFC Dichlorodifluoromethane \\
\hline CFD & Computational Fluid Dynamics \\
\hline CFL & Courant Friedrichs Lewy \\
\hline COPD & Chronic Obstructive Pulmonary Disease \\
\hline DES & Detached Eddy Simulation \\
\hline DPI & Dry Powder Inhaler \\
\hline ERV & Expiratory Reserve Volume \\
\hline$F E V_{1}$ & Forced Expiratory Volume in One Second \\
\hline FPF & Fine Particle Fraction \\
\hline FRC & Functional Residual Capacity \\
\hline FVC & Forced Vital Capacity \\
\hline GOLD & Global Initiative for Chronic Obstructive Lung Disease \\
\hline HFA & Hydrofluoroalkane \\
\hline IC & Inspiratory Capacity \\
\hline IRV & Inspiratory Reserve Volume \\
\hline LES & Large Eddy Simulation \\
\hline MAPP & Maximum Average Potential Price \\
\hline ODB & Ontario Drug Benefits \\
\hline PDA & Phase Doppler Anemometry \\
\hline PDE & Partial Differential Equation \\
\hline pMDI & Pressurized Metered-Dose Inhaler \\
\hline RANS & Reynolds-Averaged Navier-Stokes \\
\hline RMS & Root Mean Square \\
\hline RV & Residual Volume \\
\hline SGS & Sub-Grid Scale \\
\hline SMI & Respimat Soft Mist Inhaler \\
\hline SST & Shear Stress Transport \\
\hline TB & Tuberculosis \\
\hline TKE & Turbulence Kinetic Energy \\
\hline TLC & Total Lung Capacity \\
\hline TV & Tidal Volume \\
\hline URANS & Unsteady Reynolds-Averaged Navier-Stokes \\
\hline UV & Ultraviolet \\
\hline $\mathrm{VC}$ & Vital Capacity \\
\hline
\end{tabular}




\section{List of Symbols}

\begin{tabular}{|c|c|c|}
\hline Symbol & Definition & Units \\
\hline$\rho_{p}$ & Particle Density & $\mathrm{kg} / \mathrm{m}^{3}$ \\
\hline$d_{p}$ & Particle Diameter & $\mathrm{m}$ \\
\hline$u_{g}$ & Mean Velocity of Carrier Gas & $\mathrm{m} / \mathrm{s}$ \\
\hline$\mu_{g}$ & Dynamic Viscosity of Carrier Gas & $\mathrm{Pa} \cdot \mathrm{s}$ \\
\hline $\mathrm{L}$ & Characteristic Dimension of Obstacle & $\mathrm{m}$ \\
\hline Stk & Stokes Number & - \\
\hline $\mathrm{g}$ & Gravitational Acceleration & $\mathrm{m} / \mathrm{s}^{2}$ \\
\hline$v_{s}$ & Settling Velocity & $\mathrm{m} / \mathrm{s}$ \\
\hline$C_{c}$ & Cunningham's Correction Factor & - \\
\hline $\mathrm{k}$ & Boltzmann's Constant & $\mathrm{J} / \mathrm{K}$ \\
\hline $\mathrm{T}$ & Absolute Temperature & $\mathrm{K}$ \\
\hline$D_{B}$ & Brownian Diffusion Coefficient & $m^{2} / \mathrm{s}$ \\
\hline$\lambda$ & Mean Free Path & $\mathrm{m}$ \\
\hline$\wedge$ & Resuspension Rate & $s^{-1}$ \\
\hline $\mathrm{R}$ & Resuspension Flux & $\mu \cdot g \cdot m^{-2} \cdot s^{-1}$ \\
\hline $\mathrm{S}$ & Surface Contamination & $\mu \cdot g \cdot m^{-2}$ \\
\hline $\mathrm{u}$ & Instantaneous Flow Velocity & $\mathrm{m} / \mathrm{s}$ \\
\hline $\bar{u}_{i}$ & Average Component of Velocity & $\mathrm{m} / \mathrm{s}$ \\
\hline$u_{i}^{\prime}$ & Time Varying or Turbulent Fluctuation Component of Velocity & $\mathrm{m} / \mathrm{s}$ \\
\hline$\tau_{i j}$ & Reynolds-Stress Term & $m^{2} / s^{2}$ \\
\hline$\epsilon$ & Scalar Dissipation Rate & $1 / \mathrm{s}$ \\
\hline$\tilde{u}_{i}$ & Filtered Velocity & $\mathrm{m} / \mathrm{s}$ \\
\hline$v_{e}$ & Eddy Viscosity Coefficient & $\mathrm{Pa} \cdot \mathrm{s}$ \\
\hline$\Delta$ & Characteristic Length Scale & $\mathrm{m}$ \\
\hline$K_{s}$ & Smagorinsky Constant & - \\
\hline$y^{+}$ & Non-Dimensional Wall Distance & - \\
\hline$A^{+}$ & Van Driest Damping Constant & - \\
\hline$u_{p, i}$ & Particle Velocity Components & $\mathrm{m} / \mathrm{s}$ \\
\hline$u_{f, i}$ & Instantaneous Fluid Flow Components at Particle Location & $\mathrm{m} / \mathrm{s}$ \\
\hline$g_{i}$ & Gravitational Components & $\mathrm{m} / \mathrm{s}^{2}$ \\
\hline$R e_{p}$ & Particle Reynolds Number & - \\
\hline$\mu_{t}$ & Turbulent Viscosity & $\mathrm{Pa} \cdot \mathrm{s}$ \\
\hline$\mu$ & Molecular Dynamic Viscosity & $\mathrm{Pa} \cdot \mathrm{s}$ \\
\hline $\mathrm{P}$ & Heater Power & $\mathrm{W}$ \\
\hline$\Delta \mathrm{T}$ & Temperature Difference & $\mathrm{K}$ \\
\hline$C_{p}$ & Specific Heat & $\mathrm{J} / \mathrm{kg} \cdot \mathrm{K}$ \\
\hline$\dot{m}$ & Mass Flow & $\mathrm{kg} / \mathrm{s}$ \\
\hline $\mathrm{B}$ & Constant of Proportionality & - \\
\hline I & Transmitted Intensity & $\mathrm{W} / m^{2}$ \\
\hline$I_{0}$ & Original Intensity & $\mathrm{W} / m^{2}$ \\
\hline $\operatorname{Tr}$ & Transmittance & - \\
\hline
\end{tabular}




\begin{tabular}{ccc} 
A & Absorbance & - \\
$\epsilon_{e}$ & Extinction Coefficient & $1 / \mathrm{M} \cdot \mathrm{cm}$ \\
$\mathrm{c}$ & Concentration & $\mathrm{kg} / \mathrm{L}$ \\
$d_{c}$ & Path Length & $\mathrm{cm}$ \\
$\mathrm{TP}$ & Total Penetration & - \\
$m_{\text {spacer }}$ & Mass of Medication Deposited in Spacer & $\mathrm{mcg}$ \\
$m_{f i l t e r}$ & Mass of Medication Deposited in Filter & $\mathrm{mcg}$ \\
$B_{a, 1}$ & Bias Uncertainty of Absorbance & - \\
$S_{a, 1}$ & Random Uncertainty of Absorbance & - \\
$B_{b, 1}$ & Bias Uncertainty of Washing Procedure & - \\
$S_{b, 1}$ & Random Uncertainty of Washing Procedure & - \\
$B_{c, 1}$ & Bias Uncertainty of Flow Measurements & - \\
$S_{c, 1}$ & Random Uncertainty of Flow Measurements & - \\
$B_{d, 1}$ & Bias Uncertainty of Total Deposition & - \\
$B_{d, 1}$ & Random Uncertainty of Total Deposition & - \\
$B_{T P}$ & Total Bias Error on Total Penetration & - \\
$S_{T P}$ & Total Random Error on Total Penetration & - \\
$U_{T P}$ & Total Uncertainty on Total Penetration & - \\
$\mathrm{t}$ & $95^{t h}$ Percentile Point for Two-Tailed Student t Distribution & - \\
$\mathrm{C}$ & Courant Number & - \\
$\Delta \mathrm{t}$ & Time Step & $\mathrm{s}$ \\
$\Delta \mathrm{x}$ & Length Interval & - \\
$\mathrm{M}$ & Sample Mean & - \\
$s_{M}$ & Standard Error of Mean & \\
\hline
\end{tabular}




\section{Chapter 1: Introduction}

Asthma is one of the most common lower respiratory tract diseases and, as of 2016, is the third most common chronic disease affecting Canadians [1]. In 2011 to 2012, this chronic disease was found to affect about 3.8 million Canadians and is increasing year to year [2]. In 2010, asthma was found to be prevalent in $8.4 \%$ of the population of the United States (about 24.6 million people) [3].

Asthma can be defined by shortness of breath, chest tightness, and coughing as a result of airway inflammation due to certain exposures (such as exercise, allergens, and airborne irritants) $[3,4]$. This chronic airway disorder has a great impact on an individual's ability to breathe and, as such, can cause difficulties in daily life and in social activities. Activity limitations are common by those who suffer from asthma. Asthma attacks and breathing problems due to asthma have the potential to impact an adult's work day or a child's school day. In 2008, children aged 5 to 17 years were reported to miss 10.5 million school days in the United States due to asthma attacks. Additionally, adults age 18 and older were reported missing 14.2 million workdays due to asthma attacks [4].

Most of those affected by asthma spend their lives coping with the symptoms, however, with proper treatment, management, and understanding, many of those afflicted can live active lives. One such method of treatment is to use spacer devices, also known as add-on devices or holding chambers, to deliver medication from a pressurized metered-dose inhaler (pMDI). The original pMDI product (as described in a 
sales brochure from 3M in 1956) had an elongated mouthpiece, to allow for improved drug delivery and effectively acting as the first spacer device [5]. It is typical to see these add-ons, such as spacers, to enhance the total amount of medication delivered to the lungs during a dosage as illustrated in Figure 1.1. However, spacer devices have also been known to cause medication losses due to the deposition of medication on the walls of the spacer itself. This research has been developed to provide an understanding of the mechanisms of aerosol deposition as well as the efficiency of a large- and

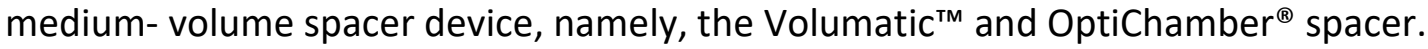

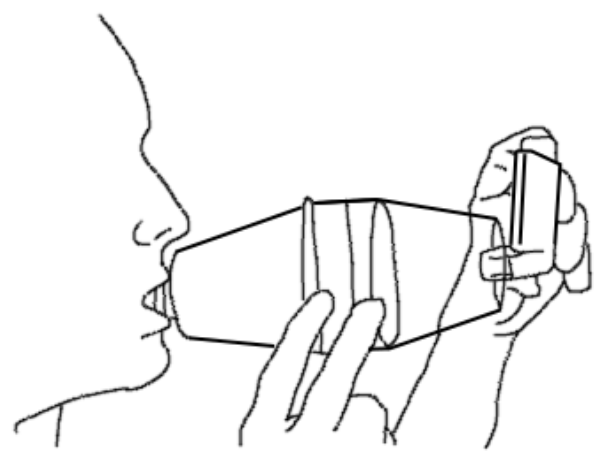

Figure 1.1: Medication delivery to patient via pMDI (Adapted from [6])

\subsection{Motivation}

Spacer devices are commonly recommended as add-on devices when using a pMDI, especially for children and seniors affected by asthma. Lung deposition from spacer devices have been found to increase or, at the very least, match the deposition from a pMDI alone [5]. It was also found that large volume spacers promote a greater lung deposition than small volume spacers $[5,7,8]$. However, an amount of medication was found to deposit on the walls of the spacer itself $[9,10]$. As such, it is important to 
understand the physics involved in this aerosol deposition to aid in the development of a truly efficient device. An efficient spacer device can be defined as one which delivers the majority of the pMDI dosage to the patient with minimal inhalation effort.

The goal of this research is to establish a better understanding of the behaviour of medication once it is released from the pMDI and enters a holding chamber. Additionally, with a comparative study of two spacers, an understanding of spacer efficiency in its use and design was to be established.

\subsection{Thesis Statement}

The current research was conducted to quantify the regional deposition and behaviour of the aerosol released from a pMDI into a medium- and large- volume spacer under a series of typical inhalation flow rates. As such, the mechanism of deposition and behaviour of the aerosol is to be quantified to provide a better understanding of how the medication can be better delivered to a patient using a spacer device. It is expected that higher flow rates will allow for a greater amount of medication reaching the patient, however, the vast majority of the aerosol should still deposit in the spacer itself. Additionally, a large volume spacer should be capable of delivering a larger amount of medication than a medium volume spacer.

This research will focus on the measurement and numerical simulation of the pMDI aerosol deposition on the walls of two spacer devices. The regional deposition of salbutamol sulphate within a spacer is studied through spectrophotometry and computational fluid dynamics (CFD). By better understanding the physics involved in this 
aerosol deposition, more efficient spacer designs or a redesign of the pMDI actuator itself may be developed.

\subsection{Research Gap}

Due to the prevalence of spacers, there is a wide diversity in the design of spacer devices. However, despite these developments, it seems that the fundamental design of spacer devices come with a lack of proper research and testing. Deposition and delivery efficiency measurements seem to be an afterthought. As such, there is minimal data studying the mechanism of aerosol deposition inside spacer devices and optimal spacer design to counteract certain deposition mechanisms. This research will build on previous literature to further an understanding of, not only spacer performance efficiency, but the underlying mechanism of aerosol behaviour in a medium- and largevolume holding chamber.

Furthermore, drug delivery from spacers have been assessed in the past using a variety of techniques including in vitro particle measurements, chemical assaying, pharmacokinetic studies, and radionuclide imaging studies $[7,11,12,13,14]$. This has created a broad array of testing protocols. As such, there appears to be a lack of a standardized protocol which demonstrates the performance of a spacer device under specific and relevant conditions. To address this, a standardized procedure was adopted from previous work, namely, Alhegagi [15] and Sprigge [16]. 


\subsection{Overview}

This thesis will be broken into five major sections. First, a background and literature survey will be presented, introducing readers to topics important to understanding aerosol drug delivery such as the respiratory system, common respiratory illnesses, and delivery devices. Second, a discussion of spray characterisation will be presented in order to provide the reader with a better understanding of the physics of an aerosol spray, as well as the numerical methods used in its characterisation. Third, the experimental protocol will be outlined, establishing the goals of the experiments, and providing a detailed explanation of the testing protocol used. Fourth, the numerical protocol will be outlined, establishing the goals of the numerical analysis as well as the procedure used to create numerical simulations of the pMDI spray analyses in a spacer device. Fifth, the results of both the experimental and numerical analysis will be presented alongside an appropriate statistical analysis. Additionally, a discussion of the results, the conclusions of the research as well as suggestions for future work will be stated. 


\section{Chapter 2: Background \& Literature Review}

\subsection{Introduction}

This section will outline several pertinent topics to the reader which are important to understand the nature of aerosol drug delivery and why it is required in the treatment of respiratory illnesses and disorders. The focus of this background information will be on drug delivery from the pMDI and spacer devices, however, other common methods of drug delivery will be introduced as well.

\subsection{Respiratory System}

The respiratory system consists of a few organs but serves as a complex biological system with the primary function of gas exchange between blood and air. Simply put, gas exchange works in two stages: inspiration and expiration. During inspiration, atmospheric oxygen is transferred to the blood through the alveolar sacs and capillaries in the lungs, and, during expiration, carbon dioxide is transferred out of the blood through the capillaries and alveoli. Aside from gas exchange, the respiratory system also plays a role in speech production, filtration, humidification, and a number of metabolic activities $[17,18]$.

The respiratory system consists of a series of organs and structures including the nose, pharynx, larynx, trachea, bronchi, diaphragm, and a pair of lungs [19]. This system can be divided into two major parts: an upper and lower airway. Further dividing the organs and structures into these two categories, the upper airway consists of those 
organs which lie outside the chest cavity, namely, the nose, pharynx, larynx, and trachea. Additionally, the lower airway consists of the organs which lie within the chest cavity, namely, the bronchi, bronchiole, and alveoli. The respiratory system is illustrated in Figure 2.1.

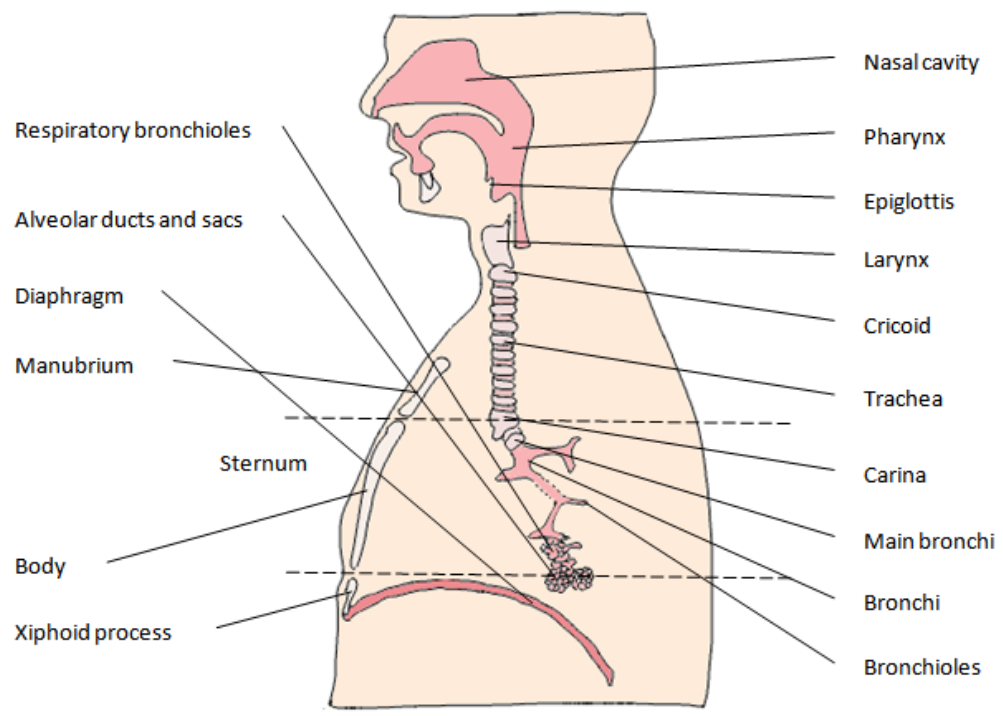

Figure 2.1: The respiratory system (Adapted from [17])

When an individual inhales, air enters the respiratory tract through the nose or mouth, and travels down the throat through the larynx and trachea and into the lungs. The lungs are the main organ involved in gas exchange, taking in atmospheric oxygen and expelling carbon dioxide. The lungs occupy most of the thorax and can be divided into the right and left lung. The left lung is divided into two lobes (upper and lower) and the right lung is divided into three lobes (upper, middle, and lower). Each lobe can be further divided into bronchopulmonary segments, each of which functions independently (as each is supplied by a unique segmental bronchus, artery, and vein) $[17,19]$. 
As air travels through the lungs, it is transported through tubes known as the main-stem bronchi. The main-stem bronchi of the lungs further divide into smaller bronchi, and then again into smaller tubes known as bronchioles. In total, there are 23 levels of bifurcations. The first generations of the branches (before 16 or 17) act to purely conduct air into the lower branches and are known as the conducting airways. Directly after the conducting airways begins the respiratory zone. At generation 16 or 17 are the terminal bronchioles, which lead to the respiratory bronchioles. The first generation of the respiratory bronchioles contain alveoli, which lead to alveolar ducts and sacs (around generation 23). Here, the walls are completely composed of alveoli [17]. These generations are demonstrated in Figure 2.2.

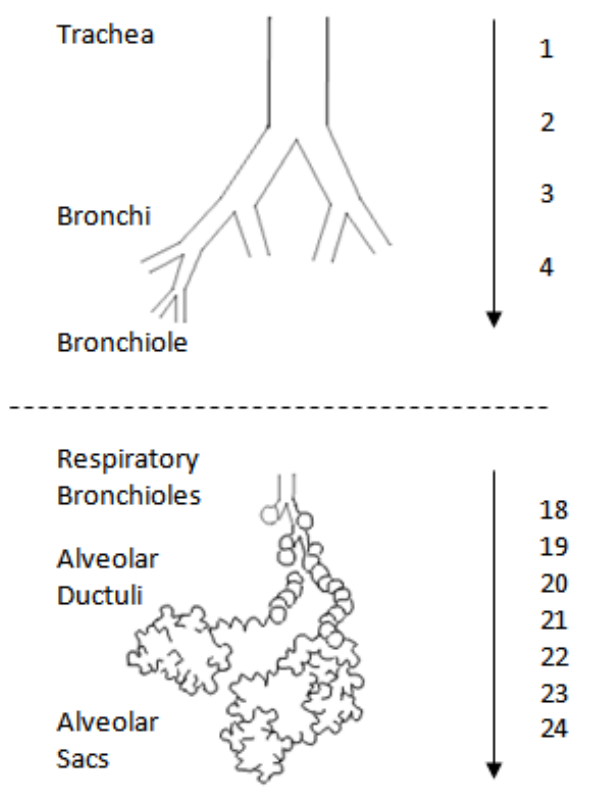

Figure 2.2: Schematic representation of the bronchial tree. Generations 1 to 16 are the conductive airways. Generations 17 to 24 are the respiratory zone (Adapted from [19]) 
Once the air has reached the respiratory zone, it will travel through the bronchioles and enter one of $\mathbf{3 0 0}$ million tiny air sacs known as alveoli to allow for gas exchange to take place. The alveoli are surrounded by a network of small blood vessels known as capillaries which have an inner diameter of about three to five microns. It is through the alveoli-capillary membrane (typically, less than a micrometer thick) which diffusion (i.e. gas exchange) takes place between the atmospheric air and blood. The membrane of the alveoli provides an exchange area of about $100 \mathrm{~m}^{2}$ and allow for the diffusion of oxygen into the blood. Once the molecule of air impacts the liquid lining which covers this membrane, it will diffuse through it to reach the plasma contained in the capillary. The molecule is then transported by the blood flow into a red blood cell, and then stored by a macromolecule known as hemoglobin. The red blood cell now possesses oxygen which it will transport away from the lungs towards wherever it may be needed $[18,19]$. This transport system of oxygen into the blood stream is demonstrated in Figure 2.3.

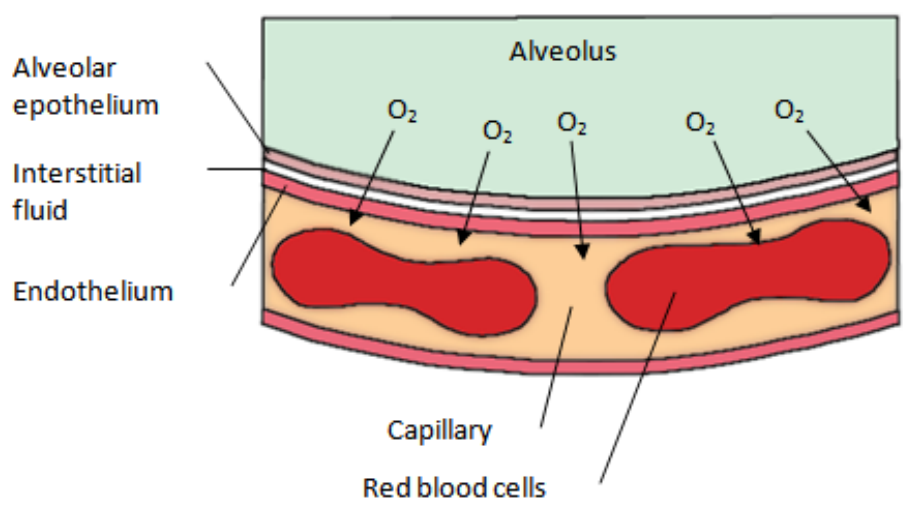

Figure 2.3: Diffusion of oxygen into the blood stream (Adapted from [17]) 
The efficiency of this oxygen exchange is dictated by a number of requirements which include the following [18]:

1) The membrane of the capillaries must be thin to allow for reasonable differences in partial pressure

2) The exchange area (i.e. boundary of the alveoli) must be large enough to allow for passive diffusion of oxygen

3) The surrounding area where the gas exchange takes place must be consistently provided with fresh air

4) The energy involved in gas exchange must be significantly smaller than the energy which it provides

For a further discussion of respiratory system physiology, please refer to Section

\section{B.1 of Appendix B.}

\subsection{Respiratory Disorders}

Respiratory illnesses are common worldwide and can affect individuals of all ages, genders, and cultures. Common repository diseases include asthma, chronic obstructive pulmonary disease (COPD), lung cancer, tuberculosis (TB), and cystic fibrosis. In 2007, Statistics Canada reported that over three million Canadians were affected by one of these five diseases. However, it is assumed this number is much larger as data 
was not available on other respiratory conditions such as influenza, pneumonia, and bronchiolitis.

More specifically, in Canada, 847,300 adults aged 35 or older were reported to be living with COPD $[20,21]$ and, in 2017 , over 4,300 people were reported to be living with cystic fibrosis [22]. In addition, as of 2018, 2,591,800 Canadians aged 12 and older had reported to be diagnosed with asthma by a health care professional. Asthma is one of the most common chronic diseases, affecting around 334 million people worldwide with $4.3 \%$ of the adult population worldwide being doctor diagnosed [23].

Respiratory diseases also have an economic impact on the Canadian health care system. As of 2007 , almost $6.5 \%$ of total health care costs were related to respiratory diseases which is approximately $\$ 12.42$ billion in direct (hospitalization, research, drugs etc.) and indirect (disability and mortality) health care costs [24].

\subsubsection{Asthma}

Asthma is an inflammatory disease which affects the large conducting airways, causing bronchial smooth muscle hyper-reactivity, inflammatory bronchial wall thickening, and increased mucus production, as illustrated in Figure 2.4. Asthma has a strong genetic component, however, environmental exposure to allergens as well as cold air and exercise can also cause asthma. As such, this condition can be classified as either allergic asthma or non-allergic asthma. Allergic asthma is commonly found in children and can be associated with eczema, rhinitis, or a food allergy and can be a result of family history. This form of asthma is triggered by an immune response as a 
result of exposure to allergens. Conversely, non-allergic asthma can occur at any age and is not an immune-system response but can be triggered by other factors such as weather changes, cold air, exercise, pollutants, and odors. Additionally, it is more common in children than adults, perhaps since younger populations have smaller airways relative to lung size. In patients over 18 years of age, asthma is more common in females (with a prevalence of around $20 \%$ higher in women than men). On the contrary, in younger patients under the age of 18 , asthma is more common in males $[23,25]$.

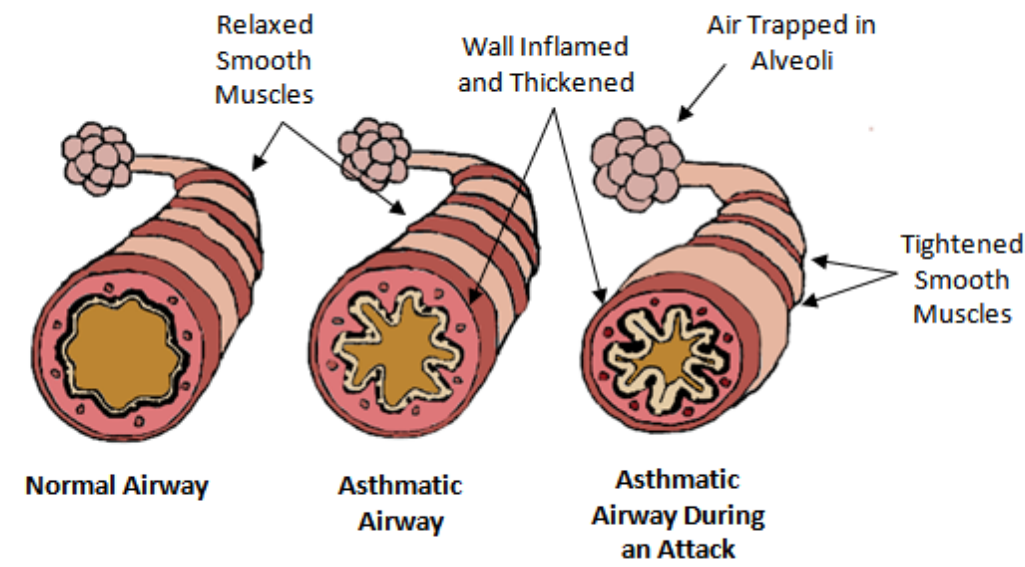

Figure 2.4: Illustration of normal airways versus those from an asthmatic (Adapted from [26])

Asthma is unique in the sense that it is intermittent, and patients can often return to normal function between asthma attacks. An attack is characterized by dyspnea (i.e. a shortness of breath), chest tightness, coughing, and wheezing (specifically during expiration). Severe attacks can also occur causing dyspnea at rest and one which interferes with conversation, as well as a peak expiratory flow less than $40 \%$ of the patients normal [25]. 
During an asthma attack one experiences bronchoconstriction, airway remodelling, and mucus production due to the release of histamine from mast cells and acetylcholine from parasympathetic neurons. In the long term, asthma can cause the permanent narrowing of affected airways.

Due to the variability of asthma symptoms, difficulties arise when practitioners only rely on the use of physical examinations. Physical symptoms may only be evident once a patient is symptomatic, these symptoms include those which indicate an airflow limitation such as a prolonged expiratory phase or wheezing during auscultation.

Patients with these physical symptoms can be assessed for the degree to which their airflow limitation exists. Excessive variability in expiratory airflow limitation is crucial to confirm an asthma diagnosis. The greater the variations in lung function, the more likely the patient is asthmatic. Due to this, spirometry is often the preferred method to objectively assess airflow limitation and variability in lung function [27].

During the diagnosis of asthma, spirometry is used to measure parameters such as forced vital capacity (FVC) and forced expiratory volume in one second $\left(\mathrm{FEV}_{1}\right)$. A typical output from a spirometer are volume-time, as demonstrated in Figure 2.5 , and flow-volume plots, as demonstrated in Figure 2.6. The ratio of $F V C$ to $\mathrm{FEV}_{1}$ provides a measure of the airflow obstruction. A healthy adult will have a ratio greater than 0.75 to 0.80 and, a healthy child would have a ratio of 0.90 . Any value less than this suggests an airflow limitation. However, since the symptoms of asthma can be sporadic, an asthmatic can still report healthy ratios as they sometimes might not exhibit an airway 
obstruction. As such, spirometry should be repeated, typically when a patient is symptomatic [27].

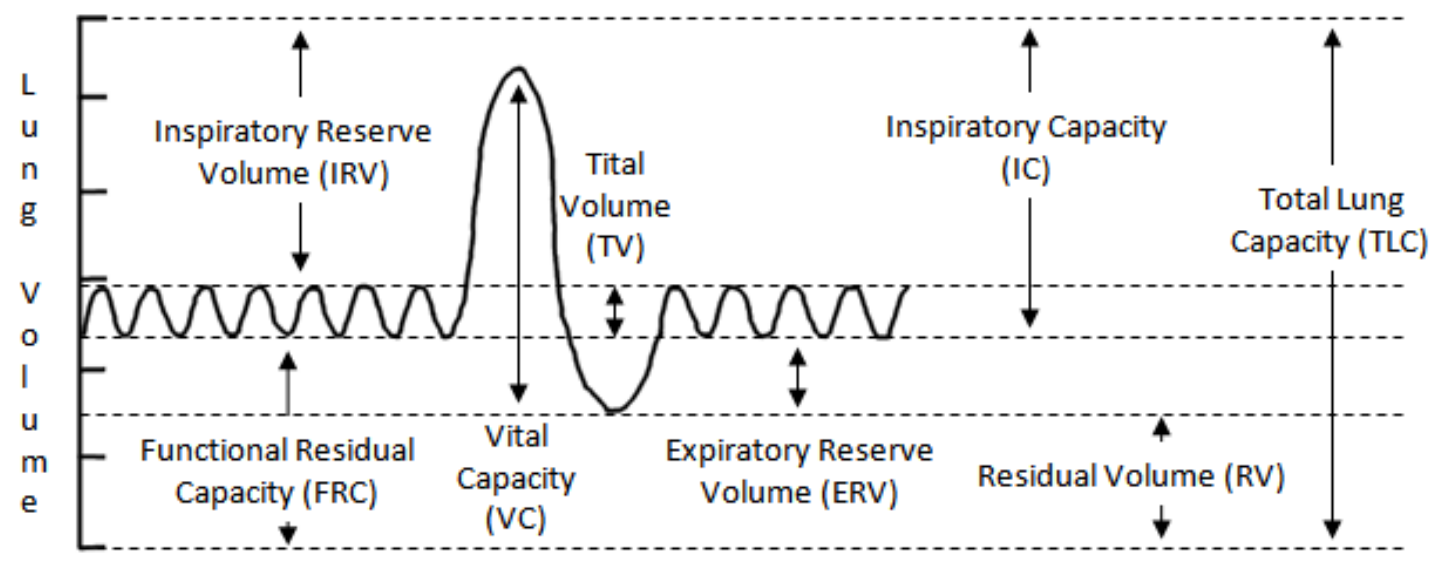

Figure 2.5: Lung capacities typically measured by a spirometer (Adapted from [28])

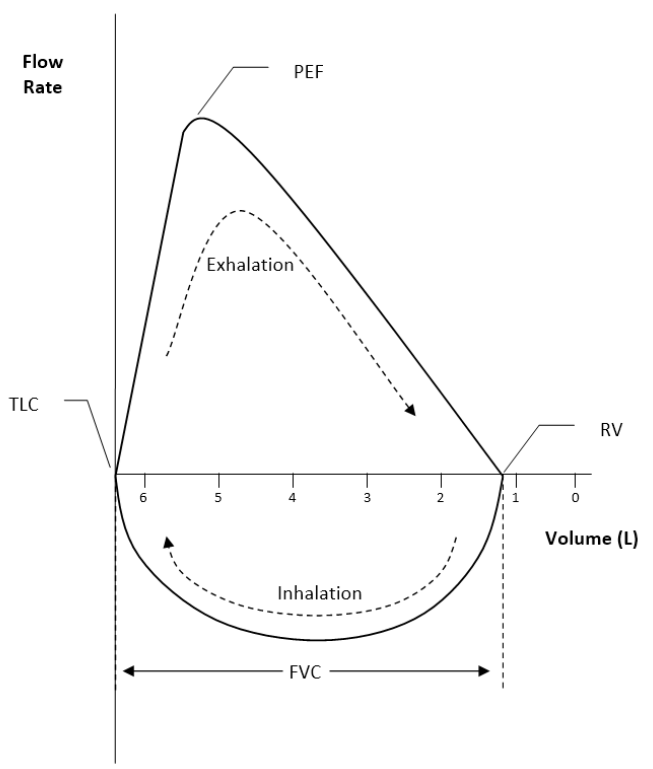

Figure 2.6: Example flow volume plot from spirometer measurements

In addition to the ratio of FVC to $\mathrm{FEV}_{1}$, obtaining objective information about the excessive variably in expiratory lung function is crucial. One such method to measure this variability is by administrating a suspected asthmatic with a rapid-acting 
bronchodilator and observing the change from baseline of $\mathrm{FEV}_{1}$. If the patient reports an increase in $\mathrm{FEV}_{1}$ of greater than $12 \%$ this could be indicative of asthma [27].

In the case that spirometric measurements are normal and the patient's symptoms and family history are still suggestive of asthma, a patient's airway responsiveness can be tested through administering a bronchoconstrictor stimuli. These can be direct airway challenges (such as histamine) or indirect (such as exercise). These challenges will observe a patient's fall in $\mathrm{FEV}_{1}$. However, positive results may be indicative of other conditions such as allergic rhinitis or COPD. Therefore, these results are most useful in ruling out asthma amongst those who are symptomatic. Other methods of diagnosing asthma include the measurement of inflammatory markers or levels of exhaled nitric oxide as well as allergy skin prick testing to identify environmental triggers of asthma [27].

\subsubsection{Chronic Obstructive Pulmonary Disease}

Chronic obstructive pulmonary disease (COPD) is a chronic respiratory disorder characterized by a not fully reversible airflow limitation and an airway inflammatory process which involves both the central and peripheral airways [29]. This airflow limitation is usually progressive and is typically associated with an inflammatory response of the lungs in response to noxious particles or gases [30]. The pathogenesis of COPD is characterized by a chronic inflammation throughout the airways as well as the parenchyma and pulmonary vasculature. Additionally, the pathologic changes which are characteristic of COPD are typically found in the central and peripheral airways, lung 
parenchyma, and pulmonary vasculature. Inflammatory cells are found to infiltrate the surface epithelium in the central and peripheral airways. This chronic inflammation can cause repeated cycles of injury and repair of the airway wall, resulting in a structural remodeling of the wall (with increased collagen content and scar tissue formation). This structural remodeling results in a fixed airway obstruction [30]. Additionally, COPD can result in the loss of elastic recoil of the parenchyma which progressively declines $\mathrm{FEV}_{1}$ as well as inadequate lung emptying on expiration [31].

Patients who exhibit symptoms such as cough, sputum production, or dyspnea are considered to be at risk of COPD. Diagnosis of COPD is confirmed through spirometry. Typical tests include determining $\mathrm{FEV}_{1}$ under the presence of a postbronchodilator. If this $\mathrm{FEV}_{1}$ is less than $80 \%$ of the predicted value and the patient exhibits a $\mathrm{FEV}_{1} / \mathrm{FVC}$ ratio of less than $70 \%$, this confirms that there is a presence of an airflow limitation which is not fully reversible. COPD can be classified in four stages by severity based on these two spirometer tests as demonstrated in Table 2.1.

Table 2.1: Characteristics of each stage of COPD (Adapted from [30])

\begin{tabular}{ccc} 
Stage & Spirometry Values & Symptoms \\
\hline 0: At Risk & Normal Values & Cough, sputum production \\
I: Mild COPD & $F E V_{1} / F V C$ ratio less than $70 \%$ & \\
$F E V_{1}$ greater than $80 \%$ & With or without symptoms \\
II: Moderate COPD & $F E V_{1} / F V C$ ratio less than $70 \%$ & \\
& $F E V_{1}$ between $30 \%$ and $80 \%$ & With or without symptoms \\
III: Severe COPD & $F E V_{1}$ less than $30 \%$ (or presence of symptoms) & $\begin{array}{c}\text { Respiratory failure or clinical signs of } \\
\text { right heart failure }\end{array}$ \\
\hline
\end{tabular}


The prevalence of COPD is typically higher in countries where cigarette smoking was or is still common, whereas, those which smoking is less common tend to have a lower prevalence of COPD [30]. In 2018, approximately 840,000 Canadians reported being diagnosed with COPD by a health professional [21]. COPD has been found to be more common in men than women and the risk factors for development typically are associated with tobacco smoking, exposure to indoor air pollution (such as biomass fuel), and physical inactivity.

The Global Initiative for Chronic Obstructive Lung Disease (GOLD) has suggested a four-stage management plan for COPD to prevent disease progression, relieve symptoms, and reduce mortality. These steps are as follows: (i) assess and monitor disease, (ii) reduce risk factors, (iii) manage stable COPD, and (iv) manage exacerbations. In brief, Table 2.2 outlines the characteristics involved in each stage. Methods of inhalation therapy are further discussed in Section B.2 of Appendix B. 
Table 2.2: GOLD COPD management plan details (Adapted from [30])

GOLD COPD Management Plan Stages

I: Assess and Monitor Disease

II: Reduce Risk Factors

III: Manage Stable COPD

IV: Manage Exacerbations
Characteristics

(i) Patients demonstrating symptoms of COPD tested

for airflow limitation using Spirometry.

(i) Reduce exposure to tobacco smoke, occupational dusts, chemicals and air pollutants.

(i) Apply a stepwise increase in treatment (depending on COPD severity).

(ii) Provide bronchodilator medications on an as-needed or regular basis to management COPD symptoms.

(iii) Initiate exercise training program.

(i) Exacerbations may indicate infection of the tracheobronchial tree or exposure to air pollution.

(ii) Treat acute COPD exacerbations with inhaled bronchodilators, theophylline and glucocorticosteroids.

\subsection{Delivery Devices}

In order to deliver inhaled drug formulations for the treatment of asthma, a device is required to create an aerosol. An aerosol can be defined as a suspension of liquid or solid particles in a carrier medium (usually a gas). Delivery of drugs directly to the lungs results in a more targeted pulmonary therapy, reducing systemic adverse effects. There are a variety of devices which are used to create an aerosol from a drug formulation. Each device usually requires specific inhalation techniques for proper treatment which can be seen as a disadvantage to inhaled drug therapies [32].

\subsection{1 pMDIs}

Pressurized metered-dose inhalers (pMDIs) are one of the most common delivery devices for inhalation drug therapy and were first conceptualized in 1955 by a three-person team consisting of George Maison, Charles Thiel, and Irving Porush. The 
development on the first pMDI started after Dr. Maison's daughter complained that her bulb atomizer leaked in her school bag, and that asthma medications should be as convenient to use as hair spray. The first clinical trials began the same year, and, in January 1956, a drug application was filed with the FDA and approved two months later. The next year, the first commercially available pMDI came onto the market [33].

The key components of a pMDI include a canister, propellant, drug formulation, metering valve, actuator, and spray nozzle as illustrated in Figure 2.7. Formulations in pMDIs can either be suspensions or solutions. The actuator nozzle is one of the critical components to aerosol formation. When the dose leaves the pMDI through the nozzle, the liquid in the propellant vapor are pulled apart by aerodynamic forces which forms a dispersion of liquid droplets. The propellant then evaporates and as a result cools the droplets. These cold droplets can then impact the back of a patient's throat causing them to abort or unsuccessfully complete the inhalation procedure due to a sudden reaction from contacting a cold substance. This is known as the Cold Freon effect [34].

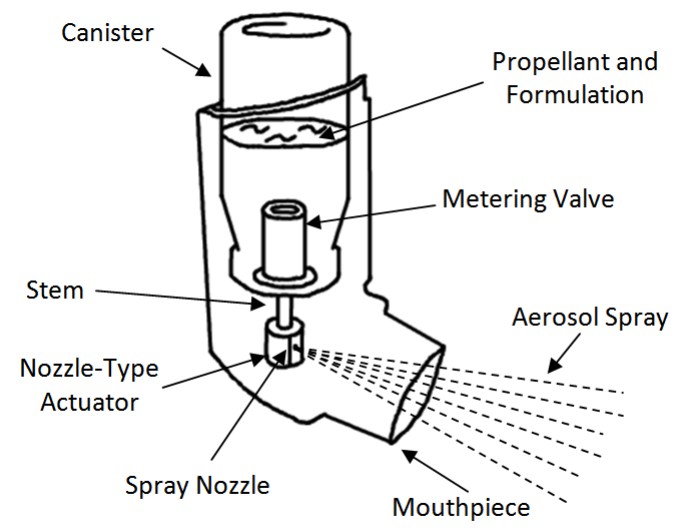

Figure 2.7: Illustration of pMDI components (Adapted from [35]) 
A propellant (or liquefied compressed gas) is necessary for pMDIs to function as it provides the driving force and energy source for atomization of the formulation during actuation. Propellants have two requirements: (i) they typically exist in two phases (liquid and saturated vapor), and (ii) they will provide the same vapor pressure regardless of how full or empty the pMDI canister is [36]. This vapor pressure is maintained to ensure a consistent dosage is provided on each actuation of the pMDI. Certain two-phase liquids (which exist as both a liquid and saturated vapor) can provide a constant dynamic equilibrium (i.e. the rate of evaporation and condensation are equal) between the liquid and vapor phases to give a constant vapor pressure as the canister is emptied.

pMDIs can be characterized into two main categories based on the type of propellant used: traditional chlorofluorocarbon (CFC) pMDIs and hydrofluoroalkane (HFA) pMDIs. CFC pMDIs were introduced in the mid-1950s and have undergone several improvements since the original conception. Albuterol (also known as salbutamol) pMDIs historically used CFC trichlorofluoromethane (CFC-11) and dichlorodifluoromethane (CFC-12). These CFCs were recognized as potent ozone depleting substances and, as of December $31^{\text {st }}, 2008$, have been progressively phased out from the market under the terms of the Montreal Protocol on Substances that Deplete the Ozone Layer [33].

During the run of CFC pMDIs, they provided a lightweight, portable, and multidose inhaler device for the treatment of asthma. Additionally, these pMDIs were able to provide constant dosing throughout their life. The traditional pMDI was also a low-cost 
solution, costing less than two dollars to produce [33]. Despite these advantages, CFC pMDIs also posed a number of disadvantages. These inhalers were specific to certain drugs and doses making them limited in use. Additionally, the CFC pMDI required initial and frequent priming before administrating a dose which lead to drug waste. Using this pMDI beyond the number of doses also resulted in a "trailing-off" effect, which could result in negligible doses. This issue was compounded by the fact that the pMDI did not contain a dose-counter and relied on the patient to count the number of doses throughout the life of the inhaler. The largest limitation of this PMDI was the inconsistent dosing which occurs with improper technique. These aerosol devices (as many do) required the patient to inhale with a slow, deep inhalation to maximize drug deposition in the airway followed by a breath-hold which allowed for the drug particles to undergo sedimentation. Any deviation from this technique would result in a lower administered dose [33].

After the ban of CFCs, new formulations were required for pMDIs. This resulted in several non-CFC pMDI versions of previous CFC pMDIs including albuterol. Amongst these new formulations were HFA pMDIs which required alterations to the PMDI design, including a smaller aperture of the metering valve (i.e. a reduction of the nozzle diameter) to produce a finer particle size. An additional modification was that ethanol was added as a cosolvent to increase solubility instead of using a surfactant. These surfactants were historically used in CFC pMDIs to increase drug solubility in CFC solutions [36]. 
A benefit which was a result of the modified valves and propellants in non-CFC pMDIs was that the "trailing-off" effect was reduced upon the depletion of the canister. Additionally, HFA pMDIs demonstrated a lower velocity aerosol plume which, when combined with smaller particle sizes due to the smaller aperture, resulted in less oropharyngeal deposition. Both benefits made the HFA pMDI more reliable and efficient than the subsequent CFC pMDI [33].

\subsubsection{Soft Mist Inhalers}

The Respimat ${ }^{\circledR}$ Soft Mist ${ }^{\mathrm{TM}}$ Inhaler (SMI) is an inhaler developed by Boehringer Ingelheim which uses the mechanical activation of a compressed spring to generate an aerosol cloud instead of a propellant. As such, the SMI is a propellant-free inhaler. The SMI consists of a cartridge containing the medication, a spring, a capillary tube, a Uniblock nozzle, and a mouthpiece as depicted in Figure 2.8.

The SMI generates an aerosol cloud with particles at a velocity of approximately one tenth of that of the pMDI over a longer period of time. Additionally, a high proportion of the droplets released in the aerosol cloud of the SMI are less than 5.8 microns in diameter, and, as such, fall into the fine particle fraction. All these factors help in reducing oropharyngeal deposition of medication and the Cold Freon effect, as well as improving the synchronization of dose generation with inhalation [37]. Furthermore, the SMI acts to reduce the "trailing-off" effect at the end of the cartridge life by locking the actuation mechanism once the dose limit has been reached (i.e. 120 actuations). The SMI has two formulations which were approved in Canada as of 2014, 
the SPIRIVA ${ }^{\circledR}$ Respimat $^{\circledR}$ and the COMBIVENT ${ }^{\circledR}$ Respimat $^{\circledR}$ which are used to treat pulmonary disorders such as asthma and COPD [38].

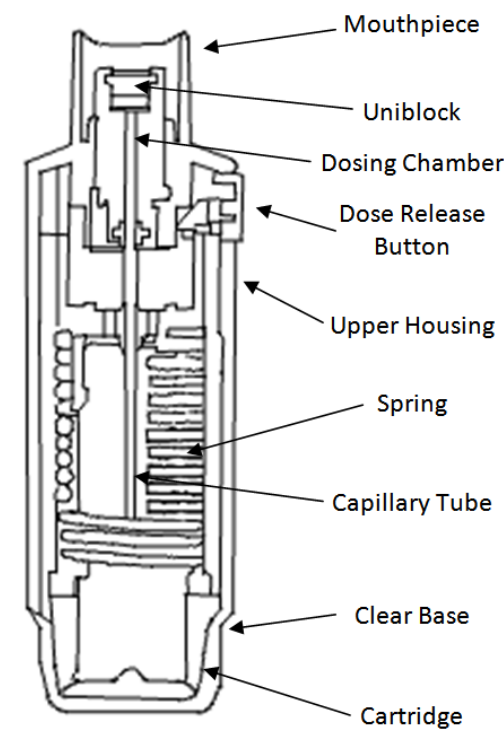

Figure 2.8: Schematic of the SMI (Adapted from [39])

The soft mist of the SMI comes from the use of a spring mechanism to force a drug solution through a two-channel nozzle. Through this mechanism, the solution is accelerated and split into two jets which collide at a controlled angle causing the solution to disintegrate into inhalable droplets. In 1991, this mechanism was first conceptualized in an early prototype consisting of a metal pump body, lever arm, a syringe acting as a solution reservoir, and a nozzle outlet which had openings of tiny holes pierced into a stainless steel disk. The lever arm was used to compress the spring and withdrew a metered dose from the reservoir. A button was then actuated to release the spring, forcing the dose through two channels which impacted near the nozzle 
outlet to create an aerosol. The nozzle design was further developed into the Uniblock in 1992 [39]. The Uniblock was an extremely fine nozzle system incorporating a rectangular glass plate (measuring $2.5 \times 2.0 \times 1.1 \mathrm{~mm}^{3}$ ) bonded to a silicon wafer as depicted in Figure 2.9 [40].

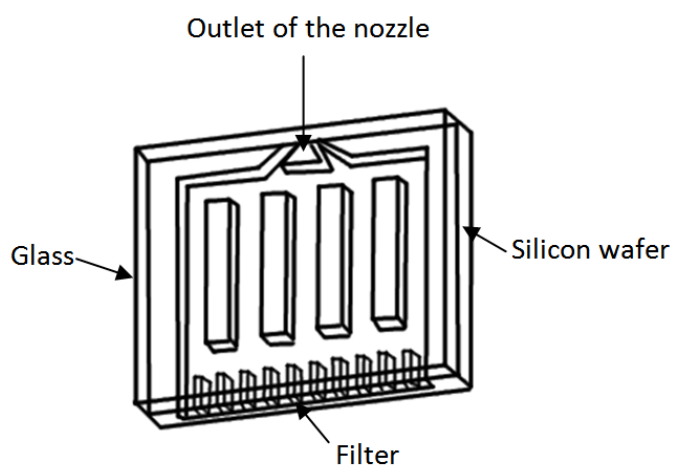

Figure 2.9: Schematic of the SMI Uniblock nozzle (Adapted from [40])

Despite the advanced development of SMI, these inhalers still pose a number of drawbacks. One of the disadvantages, that was not corrected by the SMI present in pMDIs, is that the drug cartridge still requires proper priming three times before use [39]. This can present a challenge to inexperienced and uneducated users. Additionally, a major drawback of the SMI is its cost. As of 2016, in Canada, the SPIRVIA ${ }^{\circledast}$ Respimat $^{\oplus}$ is approved to have a maximum average potential price (MAPP) of $\$ 1.05$ per puff and the pharmacy dispensing cost (for a patient without insurance) is $\$ 72.96$ for one unit (a month supply) $[41,42,43]$. This can be compared to the MAPP for the Ventolin HFA pMDI. Currently, it has a MAPP of $\$ 0.06$ per puff and the pharmacy dispensing cost for this inhaler (without insurance) is $\$ 16.95$ for one unit $[41,43]$. For comparison, the brand of pMDI used in this study, Teva-Salbutamol HFA pMDI, has a dispensing cost 
(without insurance) of $\$ 15.49$ for one unit. With insurance, Ontario Drug Benefits (ODB) can bring the costs of all these inhalers (including the SPIRIVA ${ }^{\circledR}$ Respimat $^{\oplus}$ ) to about $\$ 6.11$ per puff [43]. However, the cost difference between traditional pMDIs and the SPIRIVA ${ }^{\circledR}$ Respimat $^{\circledR}$ remains quite high (about $\$ 56$ per unit) which can pose quite a significant challenge to overcome when considering healthcare affordability.

\subsubsection{Dry Powder Inhalers}

Dry powder inhalers (DPIs) deliver a metered quantity of powder to the patient using their own inspiration. DPIs (as SMIs), illustrated in Figure 2.10, provide an alternative inhaler device which does not require the use of propellants. Through the use of a carefully formulated powder containing the drug substance, a chemically stable dry dosing system is created. These inhalers can be split up into two main categories: unit dose (or pre-metered) or multi-dose [44]. 


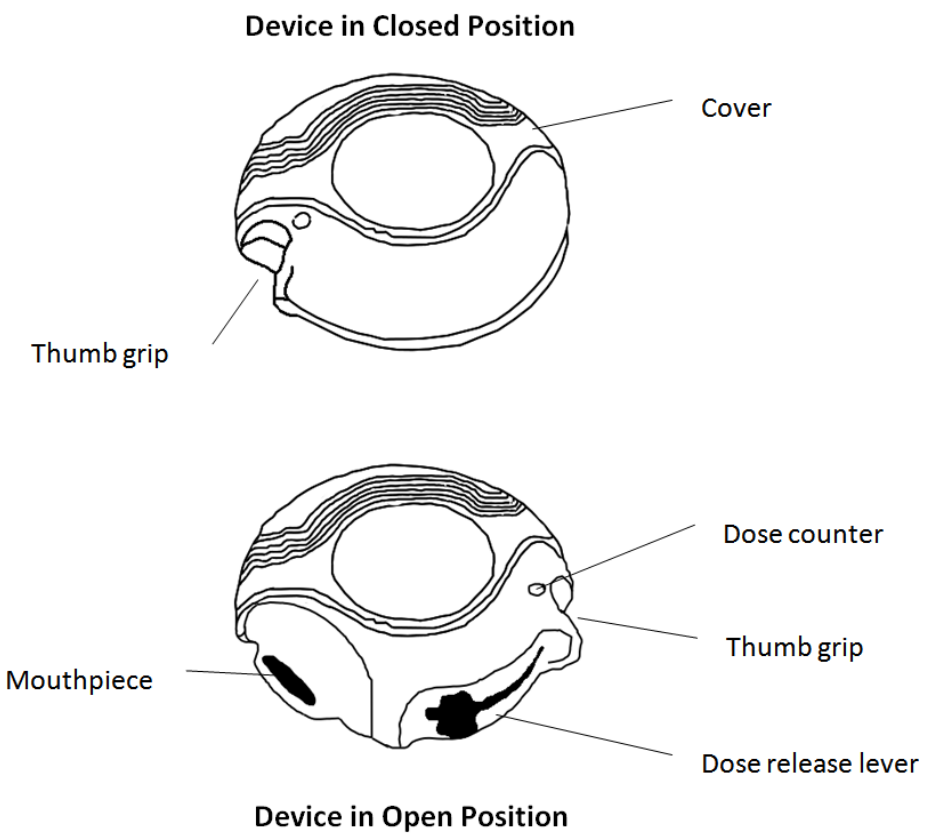

Figure 2.10: Illustration of DPI (Adapted from [45])

In order to design an efficient DPI, it is important to consider the flow and dispersion of the dry powder. The dry powder can solely consist of the drug or with the addition of a carrier material. The carrier material can act to increase the bulk of the formulation, allowing for an easier dose metering for small doses typically less than 100 micrograms. Carrier materials can also alter the flow characteristics of the formulation as well as the particle size distribution or the surface characteristics [44].

DPIs typically function by dispersing the dry powder formulation from a static powder bed. However, in order to disperse the particles, they first must be moved. To do this several mechanisms can be employed. Some DPIs use the patient's inspiratory flow to create shear and turbulence in the air (i.e. a passive dispersion mechanism). This will also introduce air into the powder bed to fluidize it. This then allows for the powder formulation to enter the patient's lungs. A major disadvantage of this passive dispersion 
is that it relies on a patient's inspiration, and if a patient cannot deeply and quickly inspire to reach the optimal peak flow rate for dispersion, the efficiency of the DPI is significantly reduced [44]. Power-assisted devices can also be employed to fluidize the powder bed (i.e. an active dispersion mechanism). These powered mechanisms can use pneumatic, impact force, or vibratory force to disperse the powder bed [46]. This fluidizing of the powder bed is illustrated in Figure 2.11. This figure demonstrates the process of a formulation being released from a metering mechanism (eg. blister disk, capsule) and then being separated through passive or active dispersion (such as patient inhalation or vibratory forces) which results in generation of large particles (depositing in the oropharyngeal region) and small particles (depositing in the pulmonary structures).

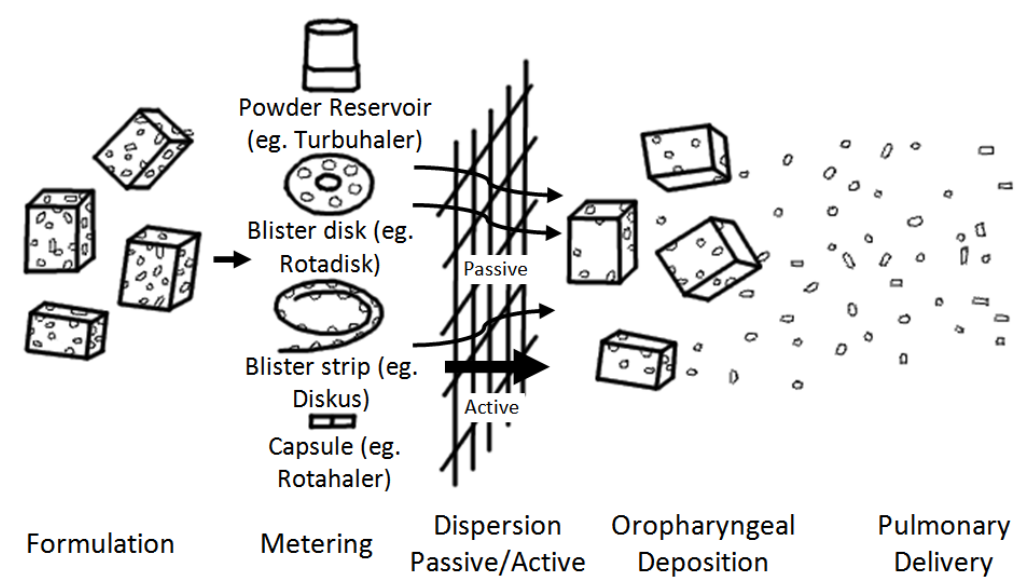

Figure 2.11: Illustration of the fluidization of a powder bed in a DPI (Adapted from [46])

The formulation must be carefully characterized and tested to understand the adhesive forces between particles which will hinder dispersion and ultimately dose effectiveness. These forces include Van der Waals forces (i.e. the electrostatic forces 
between particles, which become a dominant force for those below one micron), electrostatic forces, and surface tension of a liquid layer. These forces can be influenced by particle size and density, morphology, and surface composition. Several manufacturing and treatment process exist to limit these forces. For example, to limit the hygroscopic growth of dry particles, a lipophilic coating can be used [44].

The metering mechanism of the DPI can either use a factory controlled premetered-dose (i.e. pre-metered DPIs) or include a mechanism within the DPI so the device can meter the doses itself (i.e. multi-dose DPIs). Factory controlled pre-metered dosages are typically packaged as separate charges contained in blister reservoirs. Upon the priming of the device, the blisters are pierced, and the charge is dropped into a dosing chamber. The Diskus DPI is one such device which uses these pre-metered doses. Pre-metered doses provide an improved dose consistency as each dose will be precisely metered in the factory. For those DPIs which meter themselves, such as the Turbuhaler device, they typically contain a reservoir to contain the drug which can be dispensed into a dosing chamber by some mechanical action (such as twisting the unit). These devices allow for a large number of doses to be administered by the device while maintaining device accessibility and reducing manufacturing costs [44].

\subsubsection{Nebulizers}

Nebulizers come in many forms and provide a consistent drug delivery device. In order to nebulise a drug, it must first be incorporated into a liquid medium and then dispersed into an aerosol. The liquid medium can either be a solution or suspension 
formulation. Two main dispersion mechanisms exist, typically using either a jet (pneumatics), or ultrasonic waves.

Using compressed air or oxygen, the jet nebulizer relies on the Venturi principle (i.e. changes in air pressure to disperse the liquid formulation). By passing compressed air through a narrow orifice (or Venturi nozzle) from a high-pressure region, a lowpressure region is created which increases the gas velocity and produces a cone shaped front. This stream passes over a liquid feed tube or feeding system to create a negative pressure. This negative pressure causes the formulation (contained in a reservoir) to be withdrawn by the Bernoulli effect and formed into ligaments. These ligaments then collapse into droplets due to surface tension. The droplets then pass through a feed tube, forming a stream of entrained atomized droplets in the air flow which then flow to a mouthpiece to be inhaled by the patient. This process of atomization produces droplets around 15 to 500 microns in diameter. A larger air flow will decrease the particle size of the droplets and increase the mass flow output. Large particles (i.e. those which are not inhalable) are trapped in a baffle within the nebulizer and then are returned to the solution to be re-atomized $[47,48]$. This process is illustrated in Figure 2.12 . 


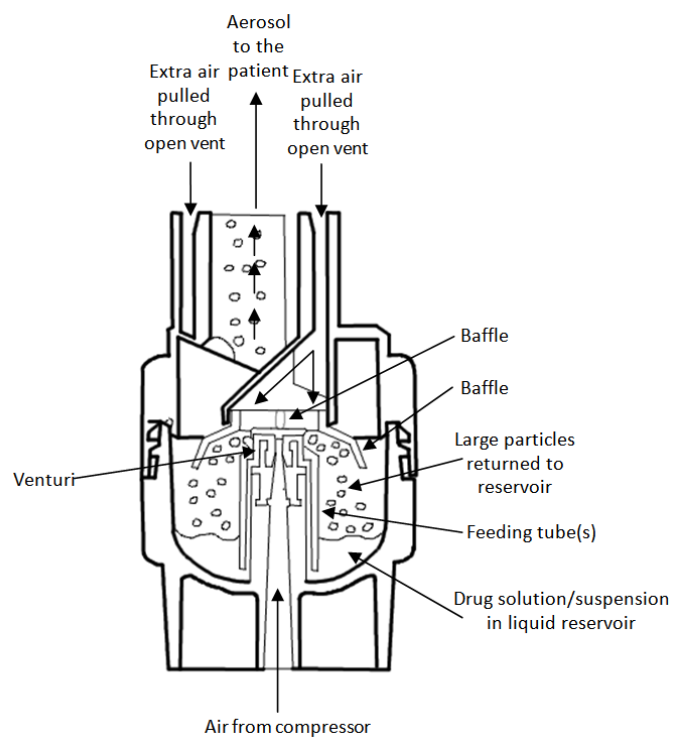

Figure 2.12: Schematic of jet nebulisation (Adapted from [48])

Ultrasonic nebulizers use a vibrating piezoelectric crystal to produce particles.

The vibrations are transmitted to the surface of the drug solution where standing waves are created. Droplets then develop from the crests of the waves and form an aerosol. The size of the droplets are inversely proportional to the power of the wave frequency. Ultrasonic nebulizers also contain baffles to remove large droplets and return them to the formulation reservoir to be re-atomized. This concept is illustrated in Figure 2.13. 


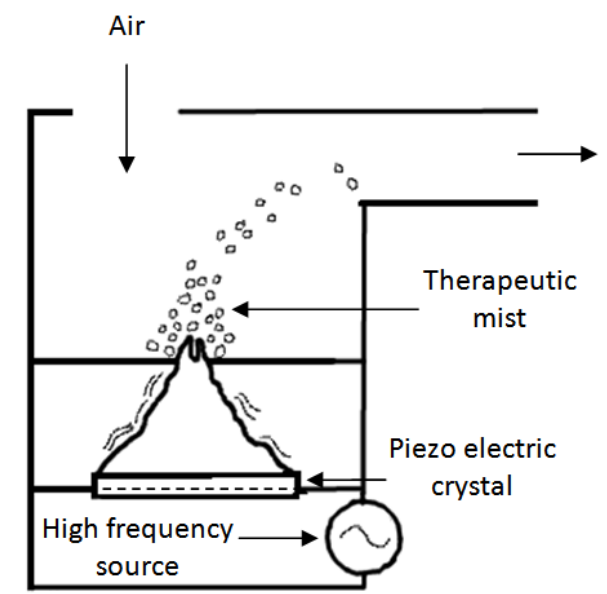

Figure 2.13: Illustration of an ultrasonic nebulizer (Adapted from [48])

Drug output from nebulizers are highly dependent on design. Particle size and nebulisation time (i.e. the time from the start of the nebulisation until continuous nebulisation has ceased) can be manipulated by altering components of the nebulizer. For example, by including extensive baffles in the nebulizer, smaller particles will be generated. However, this increases the residual volume of the drug solution (i.e. the amount of drug wastage) as larger baffles provide an increased surface area for droplet deposition which reduces the amount of drug returning to the reservoir. This in turn decreases drug output and increases the nebulisation time. A number of other factors can affect the drug output from a nebulizer including the driving gas flow, volume fill of the solution in the reservoir, solution concentration, solution temperature, and static charge [48]. 


\subsubsection{Add-On Devices}

Add-on devices (also known as spacers or extension devices) can be implemented on the end of a pMDI to correct a number of inherent limitations of these inhalers such as: high particle velocity, the Cold Freon effect, and dependence on patient technique (i.e. the requirement to have synchronicity in dose actuation and inhalation). Add-on devices can be split into three main categories: (i) tube spacer extensions to the inhaler mouthpiece, (ii) holding chambers, or (iii) reverse flow devices. A spacer can be further classified as small-volume (up to $100 \mathrm{~mL}$ ), medium-volume (100 to $350 \mathrm{~mL}$ ), and large-volume (greater than $750 \mathrm{~mL}$ ) [49]. A variety of spacers are illustrated in Figure 2.14.

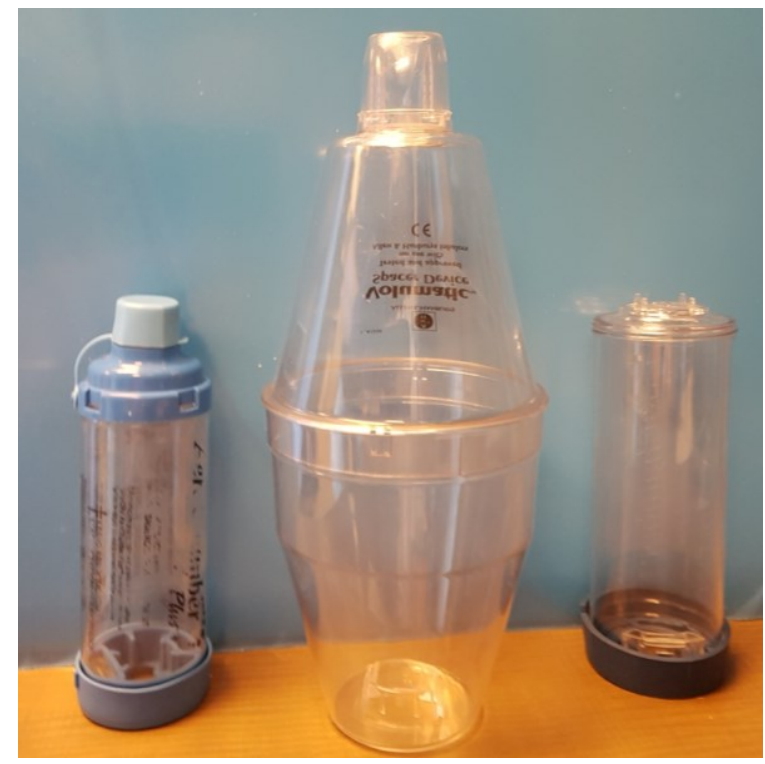

Figure 2.14: Spacer devices typically used with a pMDI (from left to right; Aerochamber $^{\circledR}$, Volumatic $^{\mathrm{Tm}}$ spacer, OptiChamber ${ }^{\circledR}$ )

Spacers can vary in size and design to accommodate different patient groups. For example, small volume spacers (such as the Babyhaler ${ }^{\mathrm{TM}}$ ) have low volume holding chambers which allow for young children to easily inhale the entire volume due to their 
limited lung capacity. Additionally, spacer devices can be manufactured out of different material including plastic (polycarbonate or polymer) and metal (steel or aluminum). Plastic spacers do not conduct electricity and can generate electrostatic charge on their inner surface which can attract aerosol particles causing a reduction of the aerosol available for inhalation. To avoid this, plastic spacers can either be primed with multiple dosages of a pMDI or by rinsing it in soapy water and left to air dry. Alternatively, metallic spacers do not accumulate electrostatic charge and can increase the fineparticle mass of the aerosol upon inhalation [49].

Despite their differing designs, each spacer acts to perform a few common functions including: the reduction of spray velocity upon actuation of the pMDI, reduction of the need to coordinate $\mathrm{pMDI}$ actuation and inhalation, a reduction of large particle fraction, and allowing for the inhalation of large doses of medication [5].

To maximize airway deposition with a pMDI, it is recommended that a patient inhales slowly (approximately $30 \mathrm{~L} / \mathrm{min}$ ) and deeply immediately after the pMDI is activated. After inhalation, the patient is advised to hold their breath for more than four seconds but optimally up to ten seconds [49]. If this technique is conducted incorrectly, it is likely that the emitted dose to the airways will be reduced and could potentially be close to zero. A spacer acts to correct for poor coordination between actuation and inhalation by holding the medication in a chamber which can be later inhaled by the patient following a short pause. This effect can be further enhanced by adding a valve to the spacer which holds the medication until the patient reaches a desired inhalation flow rate to open the valve. However, opening the valve by inspiration can pose a 
challenge to young children who are unable to generate a sufficient inspiratory pressure [49]. O'Callaghan et al. demonstrated that there is a decrease in medication delivery for a longer pause following actuation of the pMDI into a large volume spacer. At most, a 20 second delay between actuation and inhalation resulted in a reduction of drug delivery by about two thirds (about 66\%) while a pause of five seconds only reduced drug delivery by about five sixths (about 16\%) [50].

Spacer devices can also act to improve the fine particle fraction (FPF) of the aerosol. The FPF can be defined as the mass fraction of the dose emitted in an optimal size range (often less than 5 microns) for a particular inhaler [51]. By increasing the distance from the pMDI to the patient's oropharynx, the velocity of the emitted aerosol cloud is reduced by air resistance and a longer evaporation time is provided for the propellant before it is inhaled by the patient. As such, a longer propellant evaporation time reduces the particle size as the propellant is disassociated from the droplets. Additionally, the increased distance allows for the aerosol spray to warm back up after it is cooled from the propellant evaporation, reducing the Cold Freon effect. Furthermore, larger aerosol particles also are collected along the walls of the spacer, reducing oropharyngeal deposition and the amount of swallowed drug which can have unwanted systemic side-effects [49]. 


\section{Chapter 3: Spray Characterization}

In order to understand particle behavior and the physics of deposition, it is important to characterize the aerosol spray generated from a delivery device. Factors such as particle size distribution and particle velocity can affect particle entrainment, resuspension, and deposition, all of which can impact the efficiency of drug delivery methods.

\subsection{Particle Size Distribution (pMDI)}

Particle size is a crucial parameter which can have a great impact on overall drug deposition in the airways. Devices which generate an aerosol for inhalation therapy must consider particle size to determine the efficiency of the device. Fine particles, between one to five microns, typical result in a low mouth-throat deposition and a high lung deposition. However, small particles can also be exhaled more than larger particles. As a result, these fine particles are imperative to inhaled respiratory therapies. Larger particles typically deposit in the mouth and throat, and a fraction of the inhaled drug is usually swallowed [52]. The deposition potential of different particles sizes is mainly due to the deposition mechanisms which are further discussed in Section 3.2.

Usmani et al. demonstrated this by studying three different particle sizes of a highly monodisperse albuterol aerosol (1.5, 3.0, and 6.0 microns). Smaller particles were shown to achieve a greater lung deposition with $56 \%$ of the 1.5 micron aerosol depositing in the lungs compared to $46 \%$ of the 6 micron aerosol. Additionally, smaller particles had a larger distal airway penetration ( 0.79 for 1.5 micron versus 0.36 for 6.0 
micron) and more peripheral lung deposition (25\% of the 1.5 micron versus $10 \%$ of the 6.0 micron). Smaller particles were also shown to be exhaled more than larger particles with about $22 \%$ of the 1.5 micron aerosol being exhaled as opposed to $2 \%$ of the 6.0 micron aerosol. Additionally, larger particles were shown to have a greater oropharyngeal deposition with about $43 \%$ of the 6.0 micron aerosol depositing compared to $15 \%$ of the 1.5 micron aerosol [53]. The regions of deposition for various particle sizes are shown in Figure 3.1.

It is important to realize that larger particles which result in larger oropharyngeal deposition pose the risk of being swallowed by the patient, resulting in higher systemic exposure of the medication. This may cause side effects, such as, nausea and cramps, resulting in non-compliance of treatment by the patients. As such, a low lung dose with an optimal deposition pattern will result in higher efficacy and a lower systemic exposure [52].

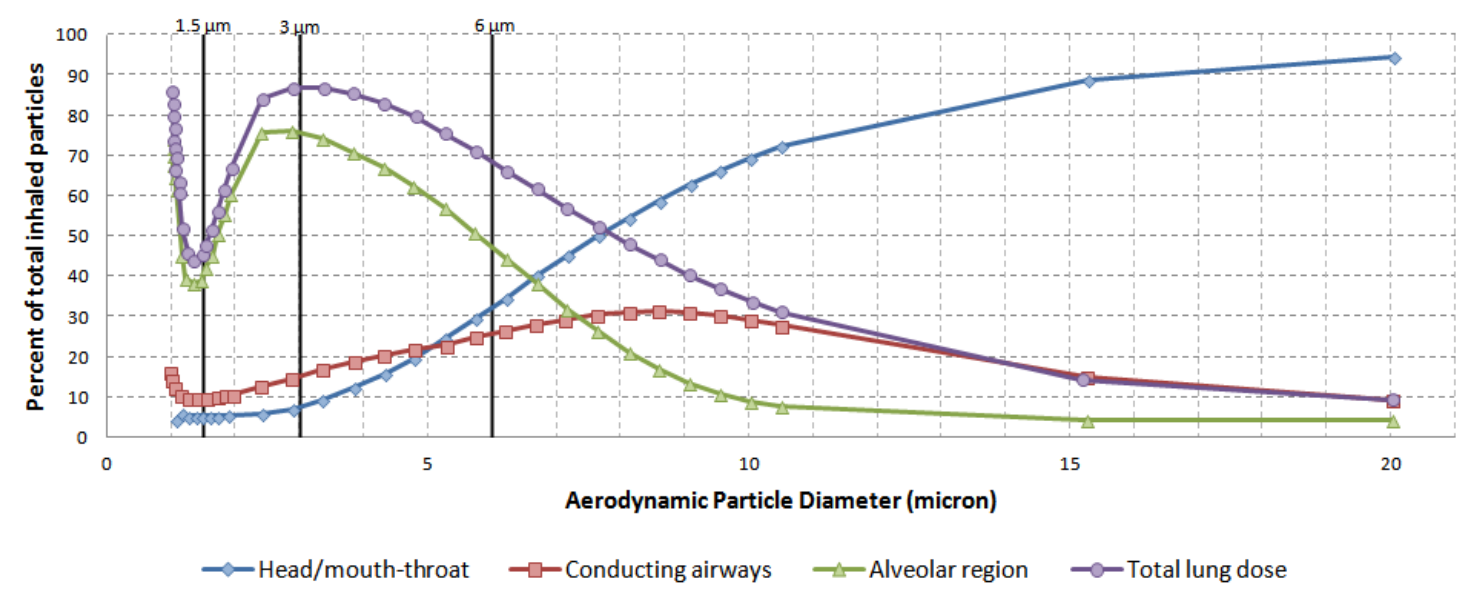

Figure 3.1: Deposition regions based on particle size (Adapted from [52]) 


\subsection{Deposition Mechanisms}

Common mechanisms of aerosol deposition include: inertial impaction, gravitational sedimentation, Brownian diffusion, turbulent mixing, and electrostatic precipitation [54]. These methods can be defined as follows.

Inertial Impaction: Mainly affects particles larger than five microns, this mechanism of deposition occurs due to sudden changes in flow direction. Due to particle momentum, particles maintain their existing trajectory and deviate from air streamlines. Additionally, large particle masses and flow rates can cause the particle to deviate from the streamline. The probability of deviation can be expressed by Stokes' number,

$$
S t k=\frac{\rho_{p} d_{p}{ }^{2} u_{g}}{18 \mu_{g} L}
$$

Where $\rho_{p}$ is the particle density, $d_{p}$ is the particle diameter, $u_{g}$ is the mean velocity of the carrier gas, $\mu_{g}$ is the dynamic viscosity of the carrier gas and $L$ is the characteristic dimension of the obstacle (typically a diameter, in the case of particles).

For the case of the salbutamol sulphate particulate (assuming a monodisperse particle size of 2.11 micron) at the inlet of a pMDI aerosol spray, the Stokes number would be on an order of magnitude of $10^{2}$.

Gravitational Sedimentation: This mechanism describes the settling of particles under the action of gravity. Sedimentation increases with particle size and particle 
residence time and can be inferred from the settling velocity of the particle under the effects of gravity. The settling velocity is the rate at which suspended particles will settle to the bottom of a fluid and is defined by,

$$
v_{s}=\frac{\rho_{p} d_{p}^{2}}{18 \mu} g
$$

Where, $g$ is the gravitational acceleration.

For salbutamol sulphate particulate with a monodisperse particle size of 2.11 micron, the settling velocity would be on an order of magnitude of $10^{-4}$.

Brownian Diffusion: Affects particles less than 0.5 micron in diameter, this mechanism describes the random motions of particles caused by collisions with gas molecules. When air velocities are low, this random motion can cause deposition. Deposition by this mechanism is proportional to the Brownian diffusion coefficient,

$$
D_{B}=\frac{C_{c} \cdot k \cdot T}{3 \pi \mu d_{p}}
$$

Where $k$ is the Boltzmann's constant, $T$ is the absolute temperature and $C_{c}$ is the Cunningham's correction factor as demonstrated in Equation (3.4).

$$
C_{c}=1+\frac{2 \lambda}{d_{p}}\left(1.257+0.4 e^{-0.55 d_{p} / \lambda}\right)
$$


Where $\lambda$ is the mean free path (i.e. the average distance travelled by a moving particle before collisions).

For salbutamol sulphate particulate with a monodisperse particle size of 0.5 micron, the Brownian diffusion coefficient would be on an order of magnitude of $10^{-5}$. Turbulent Mixing: Irregular fluctuations and mixing can occur in regions of turbulence. This can cause sporadic changes in fluid speed and trajectories of particles in both magnitude and direction resulting in deposition. Turbulent mixing is a result of flow fluctuations.

Electrostatic Precipitation: Electrically charged particles close to surfaces can induce charges on that surface. Consequently, charged particles can be electrostatically attracted to these charged surfaces. As a result, deposition of charged particles on these surfaces can be greater than that of neutral particles.

\subsection{Particle Resuspension and Entrainment}

Particle resuspension can occur when a particle is re-entrained into the flow after being deposited. Whereas, suspension or entrainment occurs when a particle was not previously deposited and remains in the flow [55]. The resuspension rate can be defined as,

$$
\Lambda\left(s^{-1}\right)=\frac{R\left(\mu g m^{-2} s^{-1}\right)}{S\left(\mu g m^{-2}\right)}
$$


Equation (3.5) is an expression of resuspension demonstrated as the fraction of a surface species removed in time where $R$ is resuspension flux and $S$ is the surface contamination. The resuspension rate is expected to vary with particle size as an increase in flow speed and particle area will result in an increase in aerodynamic lift. The adhesive force of particles would also increase with particle size due to the larger area of contact. However, a particle projecting from a viscid boundary layer is more likely to be re-suspended than a particle enclosed in stagnant or slow moving surface air [55].

\subsection{Numerical Models for Spray Characterization}

Numerical models can be implemented to model the aerosol cloud released from a pMDI. These models can help describe and understand the physics of the flow which would be difficult to obtain experimentally (such as the deposition mechanism). In order to describe the fluid flow, these models implement many different procedures to simplify the complex mathematics of the fluid flow physics. In this study, ANSYS CFX was used to generate the numerical models of the aerosol released from a pMDI.

\subsubsection{Reynolds-Averaged Navier-Stokes}

In order to model turbulent fluctuations, several turbulence models seek to model the average characteristics of turbulent flow with an additional time-varying, fluctuating component. These fluid flow equations are known as Reynolds-Averaged Navier-Stokes equations (RANS). Turbulence models based on RANS equations introduce an averaged and fluctuating component to the transport equations. In this 
case, factors such as the instantaneous flow velocity, $u$, can be expressed as an averaged component and time varying component. As an example, velocity is given by the following equation,

$$
u_{i}=\bar{u}_{i}+u_{i}^{\prime}
$$

Where $\bar{u}_{i}$ is the average component of velocity and $u_{i}^{\prime}$ is the time varying or turbulence fluctuation component of velocity. The index $i$ indicates the respective direction in a Cartesian coordinate system. The averaged component of velocity is given by,

$$
\bar{u}_{i}=\frac{1}{\Delta t} \int_{t}^{t+\Delta t} u_{i} d t
$$

Where $\Delta t$ is the time scale.

Once the dependent variables of the system have been decomposed into mean and fluctuating components, these can be substituted into the Navier-Stokes equations to yield a system of partial differential equations which governs the mean-velocity and pressure fields of incompressible turbulent flow as follows [56],

$$
\begin{gathered}
\frac{\partial \bar{u}_{i}}{\partial t}+\frac{\partial}{\partial x_{j}}\left(\bar{u}_{i} \bar{u}_{j}\right)= \\
\frac{\partial \bar{p}}{\partial x_{i}}+v \frac{\partial^{2} \bar{u}_{i}}{\partial x_{j} \partial x_{j}} \\
\frac{\partial \bar{u}_{i}}{\partial x_{i}}=0
\end{gathered}
$$


The RANS can then be obtained as,

$$
\begin{gathered}
\frac{\partial \bar{u}_{i}}{\partial t}+\bar{u}_{j} \frac{\partial \bar{u}_{i}}{\partial x_{j}}=-\frac{\partial \bar{p}}{\partial x_{i}}+v \frac{\partial^{2} \bar{u}_{i}}{\partial x_{j} \partial x_{j}}-\frac{\partial \tau_{i j}}{\partial x_{j}} \\
\frac{\partial \bar{u}_{i}}{\partial x_{i}}=0
\end{gathered}
$$

The stress term in Equation (3.10) can be expressed as,

$$
\tau_{i j}=\overline{u_{i}^{\prime} u_{j}^{\prime}}
$$

Equation (3.12) is known as the Reynolds-stress term which incorporates the effects of turbulent motions on the mean stresses. Typically, this term is unknown and must be modelled in some case-dependent manner to solve the RANS equations [56].

\subsubsection{Unsteady Reynolds-Averaged Navier-Stokes}

RANS models can also be used to describe highly unsteady flows. The application of the RANS equations in their unsteady form for this situation are known as the unsteady Reynolds-averaged Navier-Stokes equations (URANS). Equations (3.10) and (3.11) are already demonstrated in an unsteady form (i.e. they include a transient term,

$\frac{\partial \bar{u}_{i}}{\partial t}$, which can be neglected for steady state flows) and can be suitable for the calculation of unsteady transient flows as the transient term will be retained during computation. 
Additionally, URANS problems are concerned with time dependence so it is possible to see the decomposed velocity, Equation (3.6), and other flow equations, such as, Equation (3.10), ensemble-averaged and further time-averaged to see how variable such as the Reynolds-stress term, Equation (3.12), and the turbulent fluctuation of velocity vary in time, Equation $(3.6)[56,57]$. For a discussion of an alternative approach to RANS and URANS, i.e. large eddy simulation (LES), please refer to Section B.3 of Appendix B.

\subsubsection{Turbulence Modeling}

For RANS models to be solved, the Reynolds-stress tensor, Equation (3.12), must be modelled through a turbulence model, which are highly case-dependent. There are many RANS turbulence models which exist for many different situations and they can be categorized into the following classes: zero-equation models, one-equation model, twoequation models, and stress-equation models.

In zero-equation models, only a system of partial differential equations (PDEs) for the mean field are solved. One-equation models, in addition to using PDEs to solve for the mean field, incorporate an additional transport equation to calculate the turbulence velocity scale (usually in terms of the average turbulent kinetic energy, $\mathrm{k}$ ). Two-equation models involve the PDEs and transport equation of the zero and oneequation models but include another transport equation to calculate the turbulence length scale (usually in terms of the scalar dissipation rate of turbulent kinetic energy, $\varepsilon)$. Finally, the stress-equation models involve the PDEs of the zero-equation model but 
further include a number of additional transport equations for the Reynolds-stress term $\left(\tau_{i j}\right)$ and one for the scalar dissipation rate $(\varepsilon)[56]$.

Typical turbulence models seen in the application of CFD are the k- $\omega$ and $k-\varepsilon$ models which are both two-equation models. The $k-\varepsilon$ model is demonstrated to provide good results for free-shear-layer flows with small pressure gradients. Additionally, for wall bounded and internal flows, this turbulence model works well with zero and small pressure gradients but does not perform well with large pressure gradients [58]. The k- $\varepsilon$ typically predicts turbulence away from the wall boundaries well. The k- $\varepsilon$ model can be described by a turbulence energy transport equation, Equation (3.13), and an energy dissipation transport equation, Equation (3.14) [58].

$$
\begin{gathered}
\frac{\partial \rho k}{\partial t}+\frac{\partial}{\partial x_{j}}\left(\rho u_{j} \frac{\partial k}{\partial x_{j}}-\left(\mu+\frac{\mu_{\tau}}{\sigma_{k}}\right) \frac{\partial k}{\partial x_{j}}\right)=\tau_{t i j} S_{i j}-\rho \varepsilon+\phi_{k} \\
\frac{\partial \rho \varepsilon}{\partial t}+\frac{\partial}{\partial x_{j}}\left(\rho u_{j} \varepsilon-\left(\mu+\frac{\mu_{\tau}}{\sigma_{\varepsilon}}\right) \frac{\partial \varepsilon}{\partial x_{j}}\right)=c_{\varepsilon 1} \frac{\varepsilon}{k} \tau_{t i j} S_{i j}-c_{\varepsilon 2} f_{2} \rho \frac{\varepsilon^{2}}{k}+\phi_{\varepsilon}
\end{gathered}
$$

Where the right-hand side of the equation represents the production, dissipation, and wall terms, respectively.

Alternatively, the $k-\omega$ model was developed in parallel to the $k-\varepsilon$ model and describes a specific dissipation rate $(\omega)$ from one of its transport equations. This specific dissipation rate determines the scale of turbulence as opposed to the energy of the turbulence (k). The k- $\omega$ model performs well in the viscous sublayer near the wall and, as such, predicts turbulence close to the wall boundaries well [58]. The $k-\omega$ model can 
be described by a $\mathrm{k}$ (turbulent kinetic energy) scalar turbulence scale, Equation (3.15), and an $\omega$ (specific dissipation) scalar turbulence scale, Equation (3.16) [58].

$$
\begin{gathered}
\frac{\partial \rho k}{\partial t}+\frac{\partial}{\partial x_{j}}\left(\rho u_{j} k-\left(\mu+\sigma^{*} \mu_{t}\right) \frac{\partial k}{\partial x_{j}}\right)=\tau_{t i j} S_{i j}-\beta^{*} \rho \omega k \\
\frac{\partial \rho \omega}{\partial t}+\frac{\partial}{\partial x_{j}}\left(\rho u_{j} \omega-\left(\mu+\sigma \mu_{t}\right) \frac{\partial \omega}{\partial x_{j}}\right)=\alpha \frac{\omega}{k} \tau_{t i j} S_{i j}-\beta \rho \omega^{2}
\end{gathered}
$$

These two-equation turbulence models can also be demonstrated in other major formulations such as the shear stress transport (SST) k- $\omega$ turbulence model. This SST formulation takes advantage of the performance of both the $k-\omega$ and $k-\varepsilon$ models near and away from the wall boundaries. It uses the $k-\omega$ model near solid walls and the $k-\varepsilon$ model near boundary layer edges and in free-shear layers (i.e. away from the wall boundaries). This model predicts adverse pressure gradients and separating flow well, however, it does under predict the separation of the boundary layer due to adverse pressure gradients (as it does not consider the effects of transport of the turbulent stresses) [58].

\subsubsection{Particle Tracking}

If it is necessary to disperse a particulate within the domain (such as a simulation of the aerosol from a pMDI), Lagrangian particle tracking can be implemented. When RANS equations are implemented, each particle has an instantaneous velocity divided into mean and fluctuating components as in Equation (3.6). Lagrangian particle tracking 
is ideal for particles with small diameters in multiphase flow. The 3D Lagrangian equation defines the particle velocity as,

$$
\frac{d u_{p, i}}{d t}=\frac{u_{f, i}-u_{p, i}}{\tau_{p}}-g_{i}\left(1-\frac{\rho_{f}}{\rho_{p}}\right)
$$

Where $u_{p, i}$ is the particle velocity components $(\mathrm{m} / \mathrm{s}), u_{f, i}$ is the instantaneous fluid flow components at the particle location $(\mathrm{m} / \mathrm{s})$ and $g_{i}$ is the gravitational components $\left(\mathrm{m} / \mathrm{s}^{2}\right)$. The relaxation time of the particle can be expressed as follows,

$$
\tau_{p}=\frac{\rho_{p} d_{p}^{2}}{18 \mu \alpha}
$$

Where,

$$
\alpha=1+0.15 R e_{p}^{0.687}
$$

Where $\mathrm{Re}_{\mathrm{p}}$ is the particle Reynolds number.

If it is necessary to consider particle deposition due to turbulent flow, turbulent tracking can be implemented in ANSYS CFX. Turbulent tracking, also known as the random walk model, considers both the mean and fluctuating components of the instantaneous velocity. The addition of the fluctuating component results in particle dispersion in turbulent flow. To model turbulent particle dispersion, each particle is assumed to interact with eddies as it travels throughout the domain. When a particle 
interacts with an eddy, the fluctuating component of the instantaneous fluid velocity is assumed to be equal to the fluctuating velocity of the eddy, causing the fluctuating velocity to dominate. A particle will end its interaction with an eddy if the lifetime of the eddy is exceeded or the particle travels the eddy length at which point, the particle enters another new eddy.

Turbulent tracking is implemented in ANSYS CFX by selecting an eddy viscosity ratio. This eddy viscosity ratio is defined as follows,

$$
\frac{\mu_{t}}{\mu}
$$

Where $\mu_{t}$ is the turbulent viscosity and $\mu$ is the molecular dynamic viscosity.

This ratio determines how strong the influence of turbulent viscosity is compared to the dynamic viscosity. If the eddy viscosity ratio is greater than or equal to a value of five, turbulent tracking in addition to mean flow tracking will be implemented by ANSYS CFX. If this ratio is less than five, the effects of turbulent tracking will be ignored. As such, for turbulent tracking simulations, this ratio was set to the default value of five. It is important to note that adjustments to this ratio will impact how strong the influence of the turbulent viscosity is in the simulation. As such, an increase in this ratio will exaggerate the turbulence effects on particle tracks, resulting in an increase in deposition due to turbulence. 


\section{Chapter 4: Experimental Protocol}

Experiments were conducted to assess the deposition of salbutamol sulphate aerosol particles on a large- and medium- volume spacer device using spectrophotometry techniques. The large volume spacer device used was the $750 \mathrm{~mL}$ volume Volumatic ${ }^{\mathrm{TM}}$ spacer (Allen and Hanburys Ltd., London, England) as displayed in Figure 4.1.

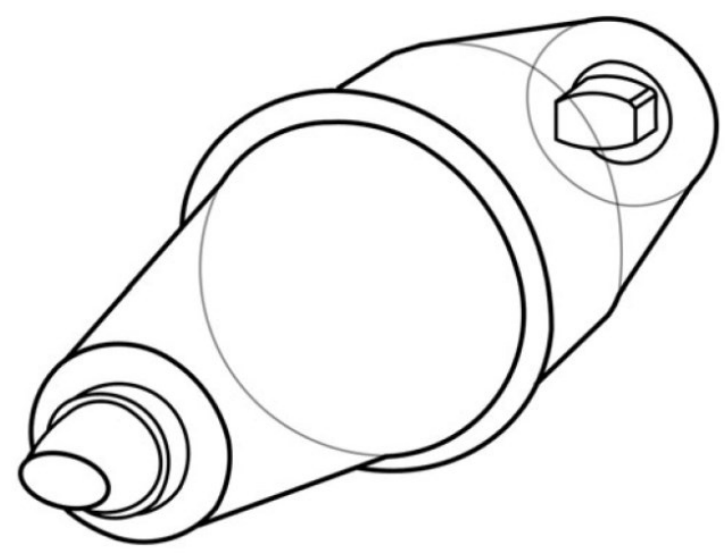

Figure 4.1: Illustration of $750 \mathrm{~mL}$ large-volume Volumatic ${ }^{\mathrm{TM}}$ spacer

Additionally, a valved $218 \mathrm{~mL}$ medium-volume spacer device was tested, namely the OptiChamber ${ }^{\circledR}$ spacer (HealthScan Products, Inc., Cedar Grove, NJ) as illustrated in Figure 4.2. Both spacers were tested using a Teva-Salbutamol HFA (TEVA Pharmaceutical Industries Ltd., Toronto, Canada) pMDI containing salbutamol sulphate. 

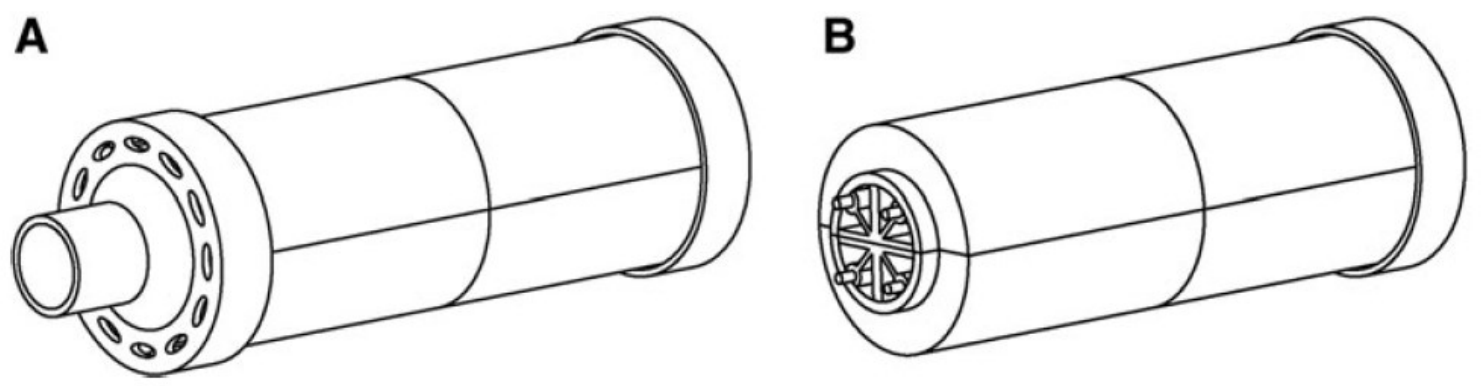

Figure 4.2: Schematic of $218 \mathrm{~mL}$ medium-volume OptiChamber ${ }^{\circledR}$ Spacer. (A) OptiChamber ${ }^{\circledR}$ with mouthpiece attached. (B) OptiChamber ${ }^{\circledR}$ with mouthpiece removed, exposing the valved portion of the spacer

\subsection{Objectives}

The main objectives of the experimental quantification of drug deposition were as follows,

I. To quantify the amount of salbutamol sulphate that remained within the spacers after pMDI actuation

II. To quantify the regions within the spacers where deposition was the most concentrated

III. To determine the effect of inhalation flow rate on the deposition patterns observed

\subsection{Equipment \& Setup}

The experimental method adopted a standardized procedure from previous work, namely, Alhegagi [15] and Sprigge [16]. This method utilized a vacuum pump to simulate three different inspiratory flow rates of 30,45 , and $60 \mathrm{~L} / \mathrm{min}$. These flow rates 
ranged from just above the breathing rate during moderate exercise to the recommended flow rate for use of a pMDI without a spacer.

The equipment used was an Agilent 8453 UV-Visible Spectrophotometer (Agilent Technologies, Santa Clara, CA), a Brooks Mass Flow Meter 58635 (Brooks Instrument, LLC., Hatfield, PA), a National Instruments Data Acquisition USB-6009 device (National Instruments Corporation, Austin, TX), and a Welch Dry Vacuum Pump 2585 B (WelchIImvac, Niles, IL). The Agilent Spectrophotometry system and the data acquisition device were connected to a computer to take instrument readings with Agilent and LabVIEW software, respectively. The selected equipment is further discussed in Section 4.2.1 through 4.2.2.

To study regional deposition, each spacer was divided into quarters (upper and lower front sections, upper and lower back sections) as demonstrated in Figure 4.3 and 4.4. To do this, rapid prototyped models of both the Volumatic ${ }^{\top M}$ and OptiChamber ${ }^{\circledast}$ spacer were produced using CREO SolidWorks software and the Dimension BST 3D ABS printer (Stratasys, Eden Prairie, MN). For the large-volume spacer, an adapter was used to fit the distal sections of the spacer to a Respirgard II filter (Vital Signs, Inc., Totowa, NJ). Additionally, the medium-volume spacer used a rubber adapter to fit the pMDI and a mouthpiece which fit the Respirgard II filter, both of which were originally part of the spacer. The filter would capture any medication leaving the spacer, which was considered analogous to the amount of medication depositing in the lungs and the oropharynx of the patient. It should be noted that the spacer devices were prototyped using ABS plastic. Left untreated, this material could result in a rougher surface then 
typically found in commercial spacer devices. Surface roughness has been found to increase particle deposition when compared to a smooth surface [59]. As such, the prototyped spacers were treated in solution to smooth the surface as much as possible. However, the increased surface roughness from the ABS plastic could still cause a slightly greater amount of deposition.

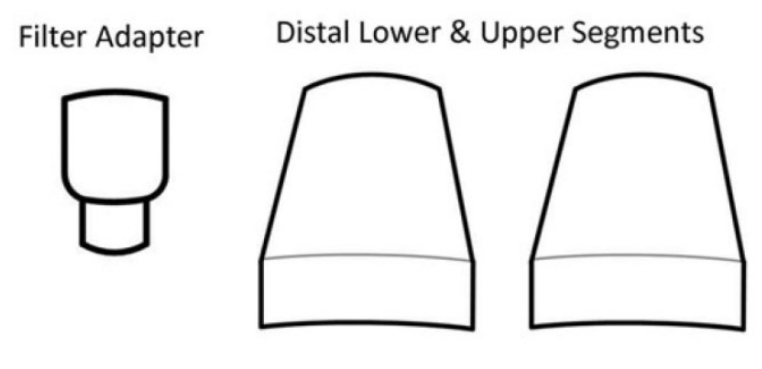

Proximal Lower \& Upper Segments

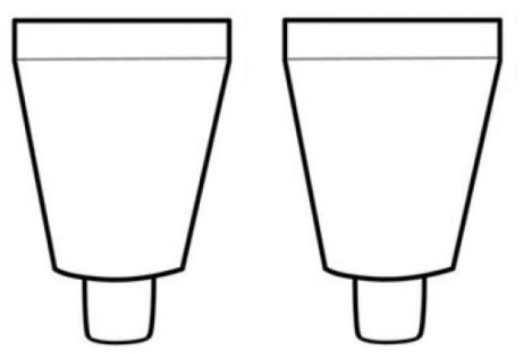

Figure 4.3: Schematic of Volumatic ${ }^{\mathrm{TM}}$ spacer divided into quarters: distal lower, distal upper, proximal lower, and proximal upper. An adaptor was used to attach the filter to the prototyped Volumatic ${ }^{\mathrm{TM}}$ spacer model 


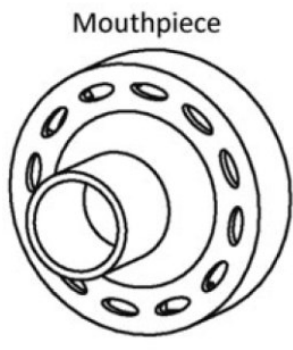

pMDI Adapter

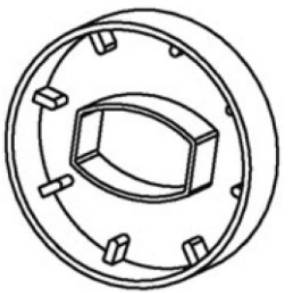

Distal Lower \& Upper Segments
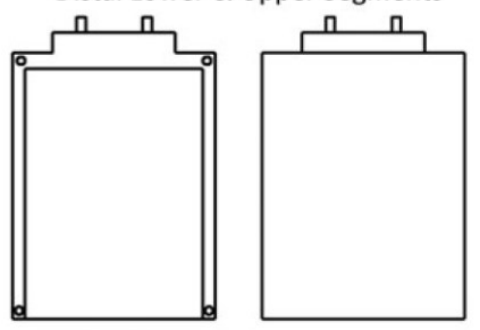

Proximal Lower \& Upper Segments
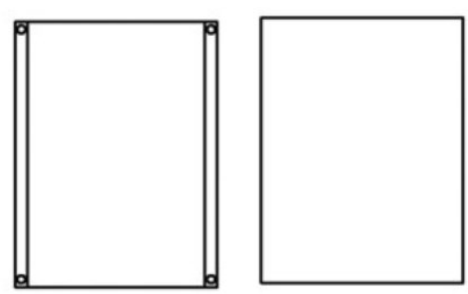

Figure 4.4: Schematic of OptiChamber ${ }^{\circledR}$ spacer divided into quarters: distal lower, distal upper, proximal lower, and proximal upper. A mouthpiece and adaptor were used to attach the filter and pMDI to the prototyped OptiChamber ${ }^{\circledast}$ spacer model

Using one foot of $12.7 \mathrm{~mm}$ diameter rubber tubing, the filter on the distal end of the spacer was attached to a flow meter. The flow meter was then connected in sequence to a flow control valve by another foot of tubing. The flow meter measured the flow rate, which was regulated by the flow control valve. Attached to the other end of the valve was a vacuum pump, which drew air through the system to draw the aerosol droplets from the pMDI into the spacer and through the filter. Figure 4.5 shows an experimental schematic of the apparatus. Experiments were carried out at the specified flow rates using the flow control valve and flow meter. 


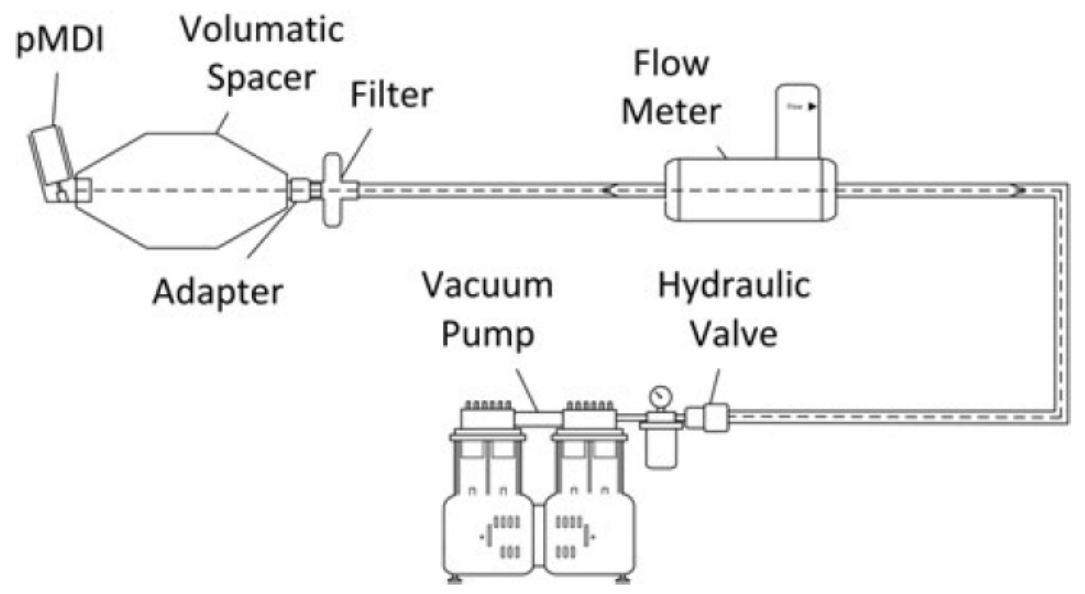

Figure 4.5: Experimental schematic of experimental setup

\subsubsection{Mass Flow Meter}

A Brooks Mass Flow Meter 5863S was used to accurately measure the flow of air through the apparatus demonstrated in Figure 4.5. This flow meter operates using a mass flow sensor to produce an electrical output signal which is linear to the measured flow rate. These units consist of three main components: a flow sensor, a control valve, and an electronic control system [60].

The flow sensor uses a thermal mass flow sensing technique which operates with two heat sensors (one upstream and one downstream). A power supply provides a heat input $(P)$ at the internal heaters of the flow meter. At a flow rate of zero, the heat reaching each temperature sensor is equal and, as such, the measured temperatures are equal. Once there is a flow through the meter, the heated upstream sensor $\left(T_{1}\right)$ is cooled by the flowing gas molecules which transport heat away from it. The downstream sensor $\left(T_{2}\right)$ remains heated and acts as a reference sensor [60]. This produces a temperature difference between the two sensors, and the upstream sensor 
is heated once again to restore the temperature balance. The electrical power required to sustain this temperature balance is indicative of a mass flow signal (i.e. a greater mass flow will cause the upstream sensor to cool much quicker). From this relationship, the gas mass flow can be determined from Equation (4.1).

$$
\Delta T=B \cdot P \cdot C_{p} \cdot \dot{m}
$$

Where $\Delta T$ is the temperature difference between $T_{2}$ and $T_{1}, C_{p}$ is the specific heat of the gas at a constant pressure, $\mathrm{P}$ is the heater power, $\dot{m}$ is the mass flow and $\mathrm{B}$ is a constant of proportionality.

Once this temperature difference is determined, a linear signal is generated which is directly proportional to the mass flow rate. A flow restrictor then provides a pressure drop across the meter which is linear with the flow rate. A control circuit is also included to sense any difference between the flow sensor signal and adjusts a solenoid control valve to increase or decrease the flow [60].

\subsubsection{UV Visible Spectrophotometer}

The Agilent 8453 UV-Visible Spectrophometer was used to measure the absorbance of salbutamol sulphate in a $20 \%$ ethanol solution. This device is capable of measuring wavelengths from 190 to $1100 \mathrm{~nm}$ [61]. Ultraviolet (UV) visible spectrophotometers measure the intensity of light passing through a solution typically housed in a cuvette. It compares this to the intensity of light before it passed through 
the sample. These spectrophotometers consist of four main components: a light source, a sample holder, a dispersive device (to separate the wavelengths of light), and a detector [62]. The basic working principle of these devices are as follows,

I. A solvent is added into a transparent, non-absorbent container such as a cuvette

II. A beam of light is emitted by the light source of the spectrophotometer which passes through the cuvette with the solvent

III. The intensity of the transmitted light is measured at different wavelengths by a detector which is placed after the cuvette and is recorded

These steps provide a blank which is used to compare to the sample. The sample is then measured as follows [62],

I. A sample is dissolved in the solvent and added to a cuvette

II. A beam of light is passed through the cuvette with the sample

III. The light is partially absorbed by the sample molecules in the solution

IV. A detector measures the transmitted light

V. The light intensity change (from the blank) at different wavelengths is measured

The detector measures the intensity of light after it passes through the sample, this is the transmitted intensity (I). This value will be lower than the original intensity $\left(I_{0}\right)$ at the light source as the there is absorption of light at specific wavelengths by the 
sample solution. When light passes through a sample solution, the transmitted intensity (I) is attenuated proportionally to the concentration of the sample [62]. The transmittance (Tr) can then be calculated using Equation (4.2).

$$
\operatorname{Tr}=\frac{I}{I_{0}}
$$

Transmittance is one of the main values used in UV visible spectrophotometry and is typically recorded as a spectrum (i.e. at each measured wavelength). From this spectrum, the different wavelengths at which the sample absorbs light can be determined [62]. Additionally, the absorbance (A) can also be determined from the transmittance as follows,

$$
A=-\log (T)
$$

The absorbance spectrum can also be represented as a function of wavelength to determine the wavelengths at which the sample absorbs light, as well as the concentration of the species from the height of the absorption peaks. The absorbance of a sample can also be determined through the Beer-Lambert law as a function of concentration and cuvette length [62]. This is demonstrated in Equation (4.4).

$$
A=\varepsilon_{e} \cdot c \cdot d_{c}
$$


Where $\varepsilon_{\mathrm{e}}$ is the extinction coefficient (describing how much the sample is absorbing at a given wavelength), $\mathrm{c}$ is the sample concentration and $d_{c}$ is the path length of the cuvette.

The Beer-Lambert law can be used to determine the sample concentration from an absorbance. To determine the unknown concentration of a sample, a calibration line must first be obtained from standard solutions of different known concentrations at a fixed wavelength. From this calibration line, the concentration of an unknown sample can be determined from its absorbance [62].

The Agilent 8453 UV-Visible Spectrophometer is a scanning spectrophotometer which measures the transmittance of the sample at each specific wavelength. The light is dispersed into individual wavelengths using a reflection grating which rotates to select each wavelength that is sent through the sample. The transmittance at a specific wavelength can then be recorded. Additionally, the whole spectrum can be recorded by continuously changing the wavelength of the light by rotating the grating [62]. This concept is demonstrated in Figure 4.6. 


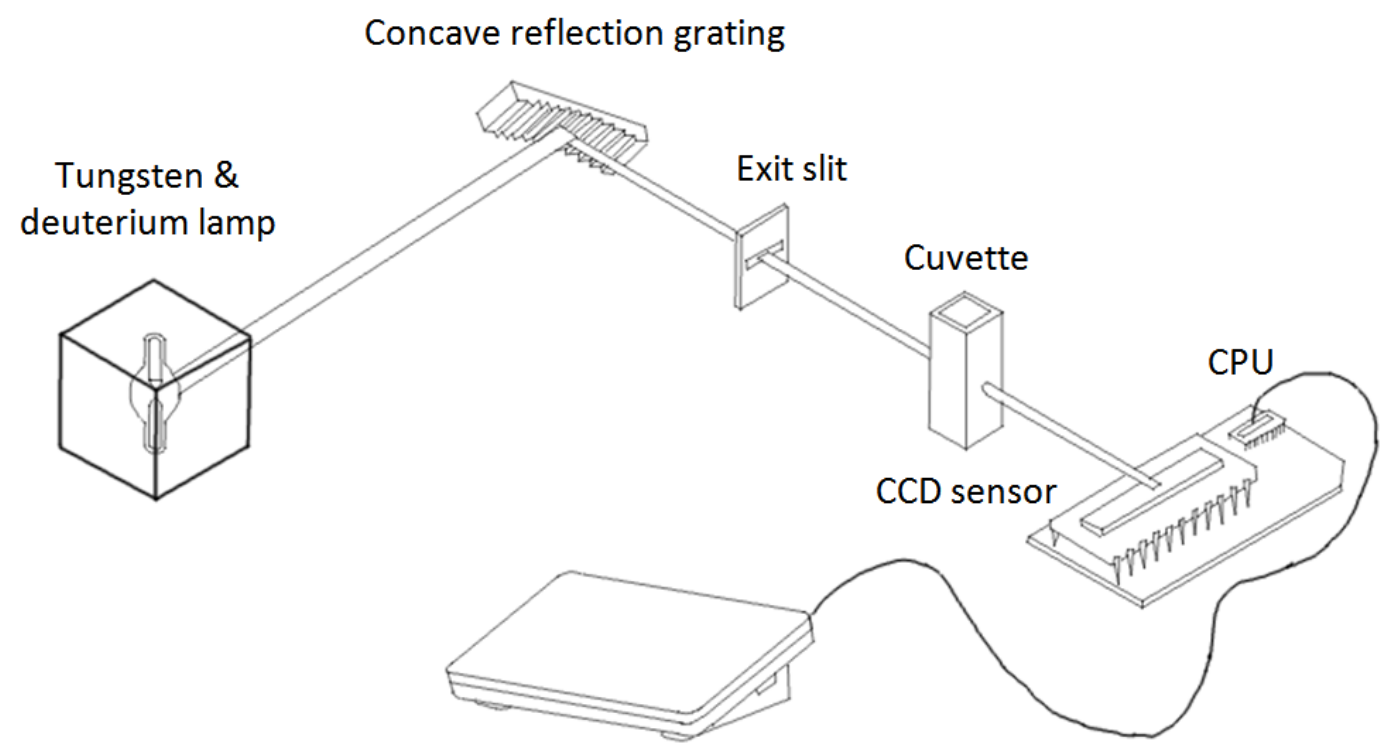

Touch screen/Terminal

Figure 4.6: Schematic of scanning spectrophotometer (Adapted from [62])

\subsection{Experimental Procedure}

The experiments conducted to assess the deposition of salbutamol sulphate in the spacer devices were performed in three parts. First, the apparatus was assembled and the pMDI was actuated into the system. Next, the apparatus was disassembled and the spacer, the filter, and the pMDI were washed to collect the deposited medication. Finally, the washings were run through a spectrophotometric analysis to determine how much medication deposited in each section. This procedure is discussed in more detail in the following subsections. 


\subsubsection{Apparatus Setup \& Medication Deposition}

Before commencing the experiments, the spacer devices were washed with dish soap and left to air dry. This minimized the losses due to suspended particles from static charge on the spacer walls as discussed by Newman [5].

Once completely dry, the quadrants were attached together using dowel pins at fixed positions and gaps were sealed using medical tape. The geometry was also wrapped in Parafilm ${ }^{\mathrm{TM}}$ to ensure no medication was released to the surroundings. The spacer and filter were attached to the flow meter using one foot of tubing and the flow meter was connected to a flow control valve by another foot of tubing. The flow control valve was used to regulate and select the desired flow rate for the experiments (i.e. either 30,45 , or $60 \mathrm{~L} / \mathrm{min})$. The flow control valve was then attached to a vacuum pump which drew air through the system in order to entrain aerosol droplets in the flow from the pMDI after actuation, so that they may move from the pMDI into the spacer and deposit in the filter. This apparatus setup is demonstrated in Figure 4.5.

The flow meter was turned on and left to run until steady flow at the desired flow rate was achieved. Steady flow was determined to be achieved once the electronic flow meter read a flow rate with minimal fluctuations. Once steady flow was reached, the pMDI was first primed by an actuation into the open air outside of the apparatus. The pMDI was then connected to the apparatus and actuated 20 times into the spacer. Between each actuation, the pMDI was vertically shaken once to ensure a consistent dosage was delivered. 


\subsubsection{Wash Procedure}

Following the 20 actuations of the pMDI into the apparatus, the actuator, the filter, and the quadrants of the spacer prototypes were disassembled in preparation for the wash procedure. The parts for each spacer were as demonstrated in Table 4.1 and illustrated in Figure 4.3 and 4.4. Each part was washed using $20 \mathrm{~mL}$ of $20 \%$ ethanol solution to determine the amount of deposition of salbutamol sulphate on each region. First, a $10 \mathrm{~mL}$ pipette was used to withdraw and dispense $20 \mathrm{~mL}$ of the ethanol solution to rinse the surface of each part. Next, a sterile cotton-tipped applicator was rinsed with the ethanol solution and used to further scrub and clean each surface. Once the surface was thoroughly rinsed and scrubbed, the tip of the applicator was again rinsed with the ethanol solution to collect any medication that may have been left in the applicator. All the washings were collected in a petri dish which were later transported to a cuvette for the spectrophotometric analysis.

Table 4.1: Washed parts for each spacer device

\begin{tabular}{cc} 
Spacer Device & \multicolumn{1}{c}{ Washed Parts } \\
\hline & (i) pMDI Actuator \\
(ii) Valve \& Mouthpiece \\
(iii) Distal Lower Segment \\
(iv) Distal Upper Segment \\
(v) Proximal Lower Segment \\
(vi) Proximal Upper Segment \\
(vii) Respirgard II Filter \\
\\
\\
(ii) Distal Lower Segment \\
(ii) pMDI Distal Upper Segment \\
(iv) Proximal Lower Segment \\
(v) Proximal Upper Segment \\
(vi) Respirgard II Filter
\end{tabular}


After the spacer prototype quadrants and the pMDI were washed, the filter was then washed. To do this, $20 \mathrm{~mL}$ of a $20 \%$ ethanol solution was passed through the filter using the pipette suction method as in Alhegagi [15] and Alhegagi and Matida [63]. This process involved coating one side of the filter with a thin layer of ethanol and drawing the solution through the opposite end using a pipette. This technique was performed multiple times to ensure the filter was thoroughly washed with $20 \mathrm{~mL}$ of ethanol solution.

\subsubsection{Spectrophotometric Analysis}

After the wash procedure was completed, the deposition of salbutamol sulphate for each flow rate could then be determined by measuring the absorbency of each wash solution.

First, as discussed in Section 4.2.2, a calibration curve was required to relate the measured absorbency to the concentration of salbutamol sulphate. To do this, $50 \mathrm{mg}$ of pure salbutamol sulphate was purchased to create a stock solution of salbutamol sulphate and $20 \%$ ethanol. Using this stock solution, the salbutamol sulphate was

diluted to known concentrations, varying from $31 \mathrm{mcg} / \mathrm{mL}$ to $500 \mathrm{mcg} / \mathrm{mL}$. These samples were run through the Agilent spectrophotometer and compared against a blank of $20 \%$ ethanol solution. Since salbutamol sulphate has a maximum absorption wavelength of 276 to $280 \mathrm{~nm}$ and a fixed wavelength node at an absorbency of $280 \mathrm{~nm}$, a wavelength of $280 \mathrm{~nm}$ was used to quantify the absorbency of all samples [15, 64, 65]. The absorption of salbutamol sulphate at $280 \mathrm{~nm}$ was then found at various 
concentrations, enough so to generate a calibration curve. This curve is demonstrated in Figure 4.7. A linear approximation was used to describe the relation between absorbance and concentration, however, this relation does appear to be non-linear at certain points. Variations in absorbance values and nonlinearity can be caused by measurement errors such as stray light [66]. The error from the spectrophotometric absorbance readings is included in the uncertainty analysis conducted in Section 4.4.

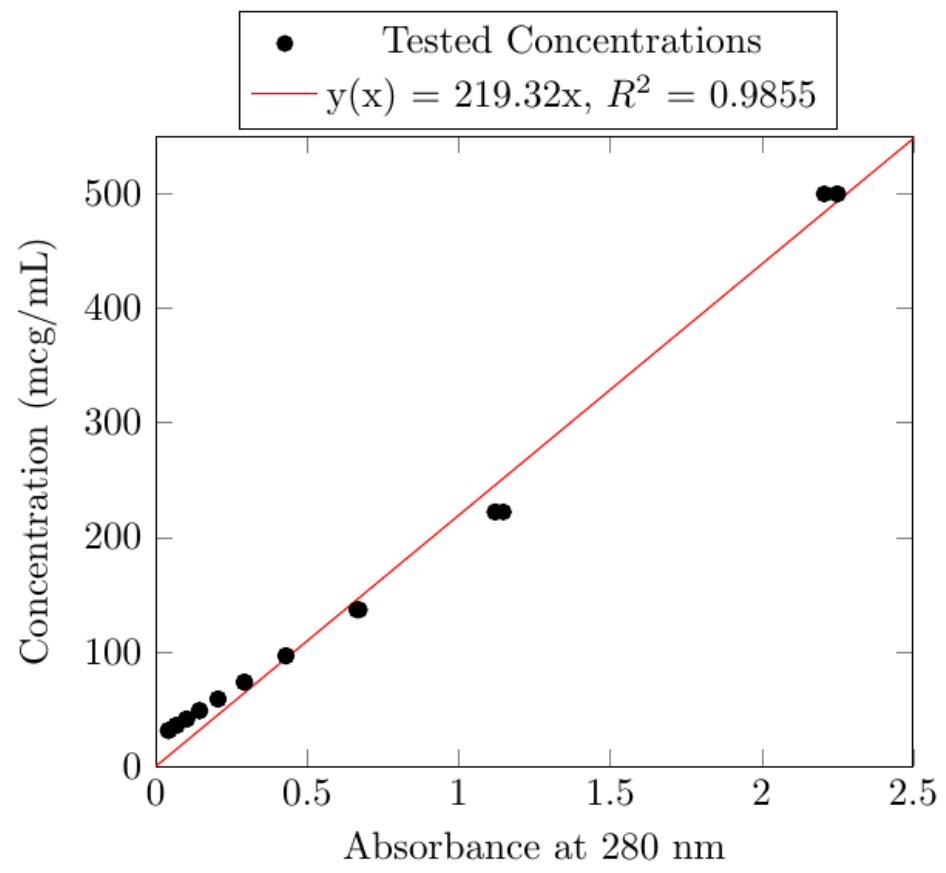

Figure 4.7: Salbutamol sulphate calibration curve for spectrophotometric analysis

After the calibration curve was generated, three absorbency readings were taken for each sample of wash solution from the various parts as demonstrated in Table 4.1. From these absorbencies, the concentration of salbutamol sulphate could then be found using the calibration curve (i.e. Figure 4.7 ) relating absorbency to concentration. Once a concentration had been determined for each part, the deposition of medication could 
then be found in micrograms since a known amount of solvent was used to dissolve the salbutamol sulphate (i.e. $20 \mathrm{~mL}$ of $20 \%$ ethanol). For reliability, these procedures were repeated at least three times for each flow rate. The repeatability criteria used for these experiments were based on the standard deviation of the average deposition measurements. If this standard deviation remained below the calculated uncertainty (discussed in Section 4.4), results were considered to have satisfied the repeatability criteria.

\subsection{Uncertainty Analysis}

An uncertainty analysis was conducted to determine the extent of the error (random and bias) on the measurements in this study. This uncertainty was calculated based on the ASME Test Uncertainty standards (PTC 19.1-2005) using five steps [67]. First, the measurement process and a "true value" were defined. Next, the elemental error sources (i.e. the sources of errors associated with measuring the true value) were listed. Third, the systematic and random uncertainty were calculated for each elemental error source. Fourth, the systematic and random standard deviations were propagated and, fifth, the total uncertainty was calculated. This procedure is demonstrated in the following subsections.

\subsubsection{Definition of the Measurement Process}

In this study, measurements were completed to determine the fraction of medication reaching the patient delivered via a pMDI and spacer device. As such, it was 
determined that the true value was the total penetration or, experimentally, the amount of medication reaching the filter. The total penetration was calculated using the following equation,

$$
T P=\frac{m_{\text {filter }}}{m_{\text {spacer }}+m_{\text {filter }}}
$$

Where TP is the total penetration, $m_{\text {spacer }}$ is the mass of medication deposited in the spacer and $m_{\text {filter }}$ is the mass of medication that penetrated (i.e. without deposition) through the spacer and deposited in the filter.

Once the true value was defined, the measurement process could be summarized as follows. Aerosol medication was delivered to the filter through a spacer device at constant volumetric flow rates of 30,45 , and $60 \mathrm{~L} / \mathrm{min}$. A percentage of the medication from 20 puffer actuations was deposited on the walls of the spacer and the remaining medication reached the filter. The mass of medication was determined through a combination of a washing procedure and absorbance measurements. The spacer and the filter were washed separately with a fixed amount of solvent (i.e. $20 \mathrm{~mL}$ of $20 \%$ ethanol). These washings were then collected and run through a UV spectrophotometer to determine an absorbance. From the Beer-Lambert curve, the concentration of medication was found from the absorbance and a deposition mass was determined. These measurements were repeated at least three times for each flow rate. 


\subsubsection{List of Elemental Error Sources}

The elemental error sources were defined as the sources of errors associated with measuring the true value (i.e. the total penetration). As described in the measurement procedure, there were three main elemental error sources associated with the determination of the total penetration. First, the absorbance of the surface washings was identified as an error source. These absorbance values were used to determine the mass of medication deposited on each surface, and, as such, if any error was present, would affect the total penetration. The second error source was identified as the surface washing procedure. This procedure involved washing the surfaces of the spacer device, pMDI, and filter with $20 \%$ ethanol. These washings were used to determine the absorbance and mass deposition on each surface. The washing procedure was completed by hand, and so, an error could be present if any of the surface remained unwashed or the surface (or washings) were contaminated in any way. Third, the measurement of the flow rate was identified as an elemental error source as errors in the measurement of the flow rate would ultimately affect the amount of medication drawn through the spacer and depositing in the filter.

\subsubsection{Estimation of Elemental Errors}

First, the bias and random uncertainties on the absorbance measurements were determined. The random uncertainty was estimated from the average standard deviation of the deposition measurements across all the trials. This resulted in a value of

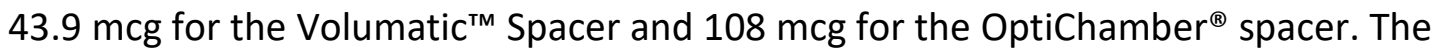


bias uncertainty was estimated from the reported bias error on the spectrophotometer of $0.01 \mathrm{~A}$ [68], which gave a value of $43.9 \mathrm{mcg}$.

Next, the bias and random uncertainties of the surface washing procedure were determined. The bias error was assumed to be zero since this was a procedure completed by hand and it was assumed there was no inherent bias in human error ${ }^{2}$. The random error was estimated based on the random error of the absorbance measure. Here, it was assumed that the random error for the washing procedure was half as that from the absorbance measurements.

Finally, the bias and random uncertainties on the flow measurements were determined. From the experimental results, there is little percent change in penetration between a flow rate of $30 \mathrm{~L} / \mathrm{min}$ and $60 \mathrm{~L} / \mathrm{min}$ (around $3 \%$ at the most). Therefore, it was assumed that the uncertainty on the flow rate had a minimal impact on total penetration. As a conservative estimate, the bias and random error were taken as half of that of the absorbance uncertainties.

Once the bias and random errors were determined for each of the elemental error sources, the overall uncertainty on the deposition was calculated using the following equations,

$$
B_{d, 1}=\sqrt{\left(B_{a, 1}\right)^{2}+\left(B_{b, 1}\right)^{2}+\left(B_{c, 1}\right)^{2}}
$$

\footnotetext{
${ }^{2}$ It was assumed that each experiment was performed without bias, that is, each experiment was performed to the best ability of a skilled researcher who had significant experience with the washing procedure used in this study. In the future, this error could be quantified by studying the differences in results which could arise if the ability and performance of the researchers were varied.
} 


$$
S_{d, 1}=\sqrt{\left(S_{a, 1}\right)^{2}+\left(S_{b, 1}\right)^{2}+\left(S_{c, 1}\right)^{2}}
$$

Where, $B_{a, 1}$ and $S_{a, 1}$ are the bias and random uncertainties on the absorbance measurements, respectively, $B_{b, 1}$ and $S_{b, 1}$ are the bias and random uncertainties of the washing procedure, respectively, $\mathrm{B}_{c, 1}$ and $\mathrm{S}_{c, 1}$ are the bias and random uncertainties of the flow rate measurements, respectively, and $B_{d, 1}$ and $S_{d, 1}$ are the bias and random uncertainties of the total deposition, respectively.

\subsubsection{Propagation of Bias and Random Uncertainties}

Once the bias and random uncertainties were calculated for each error source, the uncertainties were then propagated through to total penetration based on Equation (4.5). To do this, the following equations were used,

$$
\begin{aligned}
& S_{T P}=\sqrt{\left(\frac{S_{d, 1}}{m_{\text {filter }}}\right)^{2}+\left(\frac{S_{d, 1}}{m_{\text {spacer }}}\right)^{2}+\left(\frac{S_{d, 1}}{m_{\text {filter }}}\right)^{2}} \\
& B_{T P}=\sqrt{\left(\frac{B_{d, 1}}{m_{\text {filter }}}\right)^{2}+\left(\frac{B_{d, 1}}{m_{\text {spacer }}}\right)^{2}+\left(\frac{B_{d, 1}}{m_{\text {filter }}}\right)^{2}}
\end{aligned}
$$

Where $\mathrm{B}_{\mathrm{TP}}$ is the total bias error on the total penetration and $\mathrm{S}_{\mathrm{TP}}$ is the total random error on the total penetration. 


\subsubsection{Calculation of Total Uncertainty}

Once the uncertainties were propagated based on the total penetration, the total uncertainty was calculated combining both the bias and random uncertainty. To calculate this total uncertainty, the following equation was used,

$$
U_{T P}= \pm \sqrt{\left(B_{T P}\right)^{2}+\left(t S_{T P}\right)^{2}}
$$

Where $U_{T P}$ is the total uncertainty on the total penetration and $t$ is the $95^{\text {th }}$ percentile point for a two-tailed Student $t$ distribution. In this case, the $t$-value was determined to be a value of 2 from a t-table with 59 degrees of freedom (i.e. with $20 \mathrm{pMDI}$ actuations per trial and three trials, $n=60$ ).

Using Equation (4.10), the total uncertainty of the deposition measurements was determined to be $9.63 \%$ for the Volumatic ${ }^{\mathrm{TM}}$ spacer, and $11.35 \%$ for the OptiChamber ${ }^{\circledR}$ spacer at a $95 \%$ confidence interval. The values used in this uncertainty analysis are listed in Table 8.1 in Appendix A. 


\section{Chapter 5: Numerical Protocol}

The commercial software package, ANSYS CFX was used to conduct the numerical analysis of medication deposition inside the spacer devices. This software was selected due to its ability to conduct Lagrangian particle tracking and provide shear stress transport (SST) turbulence models. Steady state and transient simulations were conducted for each spacer device at a flow rate of $30 \mathrm{~L} / \mathrm{min}$.

\subsection{Geometry}

The geometry of the Volumatic ${ }^{\mathrm{TM}}$ and OptiChamber ${ }^{\circledR}$ spacer as well as a pMDI was generated in ICEM CFD as displayed in Figures 5.1 and 5.2. The dimensions of the Volumatic ${ }^{\mathrm{TM}}$ spacer were reproduced with a total length of $230 \mathrm{~mm}$ and a maximum diameter of $90 \mathrm{~mm}$. Additionally, the OptiChamber ${ }^{\circledR}$ was reproduced with a total length of $174 \mathrm{~mm}$ and a maximum diameter of $44 \mathrm{~mm}$. The outlet of the Volumatic ${ }^{\mathrm{TM}}$ spacer was simplified by creating a straight (rather than curved) outlet to match the experimental spacer prototype. Just as in the experiments, the geometry was split into quadrants to study the regional deposition. 


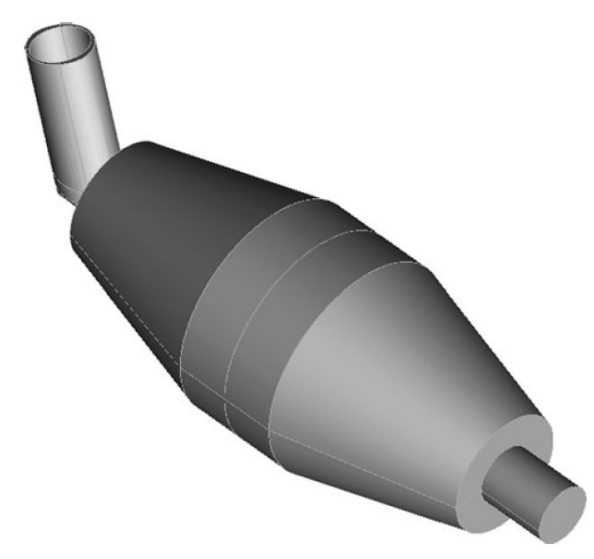

Figure 5.1: 3D render of the Volumatic ${ }^{\mathrm{TM}}$ spacer and pMDI using ICEM by ANSYS

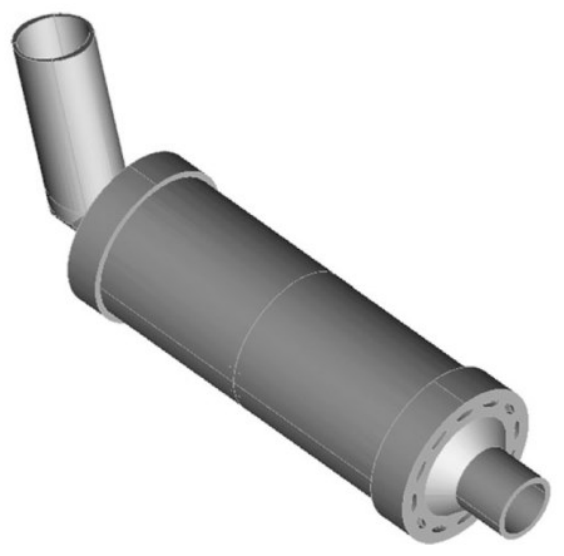

Figure 5.2: 3D render of the OptiChamber ${ }^{\circledR}$ spacer and pMDI using ICEM by ANSYS

\subsection{Mesh}

An unstructured mesh for both spacer devices were created as shown in Figure 5.3 and 5.4. The grid consisted of tetrahedral elements as well as five layers of prism elements to capture detail near the deposition surfaces of the spacer devices. The height of the first prism layer was about $0.015 \mathrm{~mm}$ to ensure a dimensionless distance from the wall (i.e. $\mathrm{y}^{+}$) of one. In order to conduct a grid convergence analysis, the unstructured grids of the Volumatic ${ }^{\top M}$ spacer ranged from $\sim 2$ million elements to $\sim 6$ 
million elements. Similarly, the unstructured grids of the OptiChamber ${ }^{\circledast}$ ranged from $\sim 2$ million elements to 6 million elements.

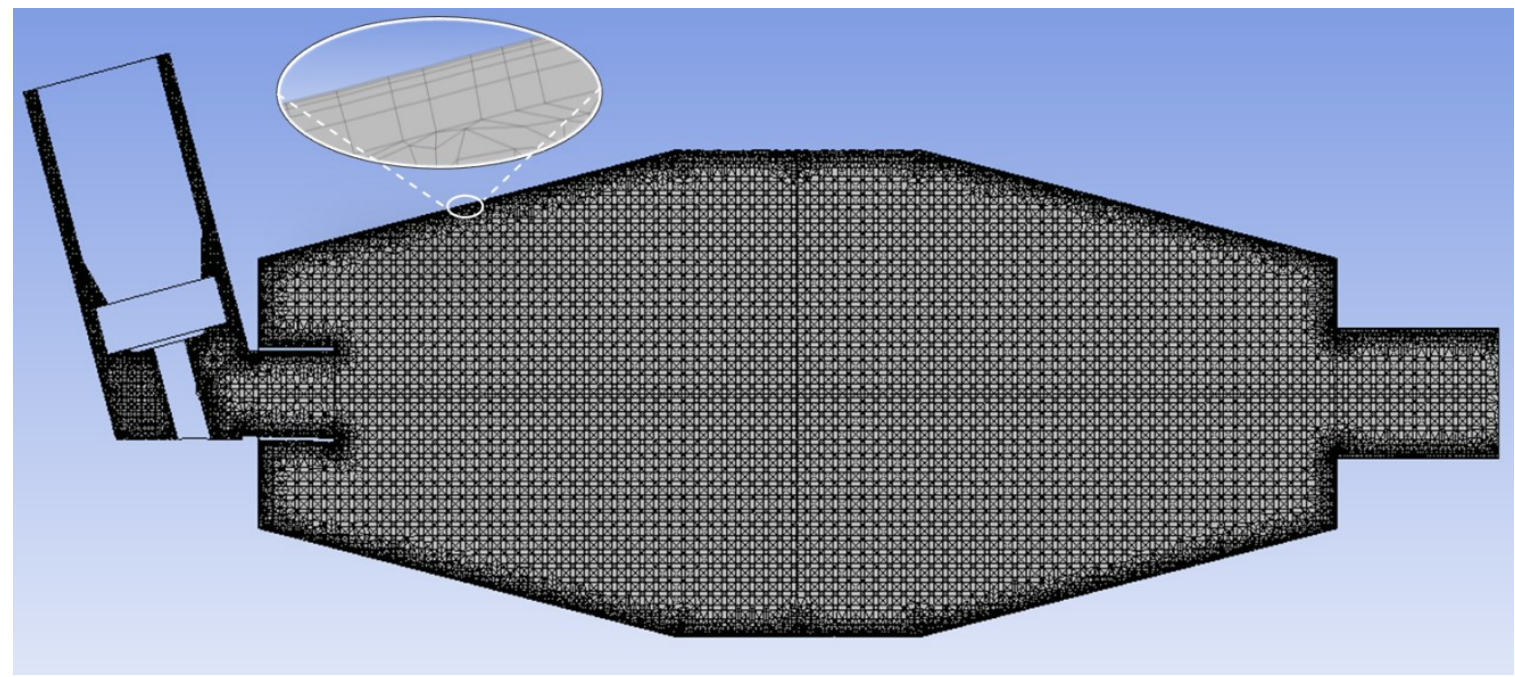

Figure 5.3: Unstructured mesh of Volumatic ${ }^{\mathrm{TM}}$ spacer geometry (6 million elements with prism layers)

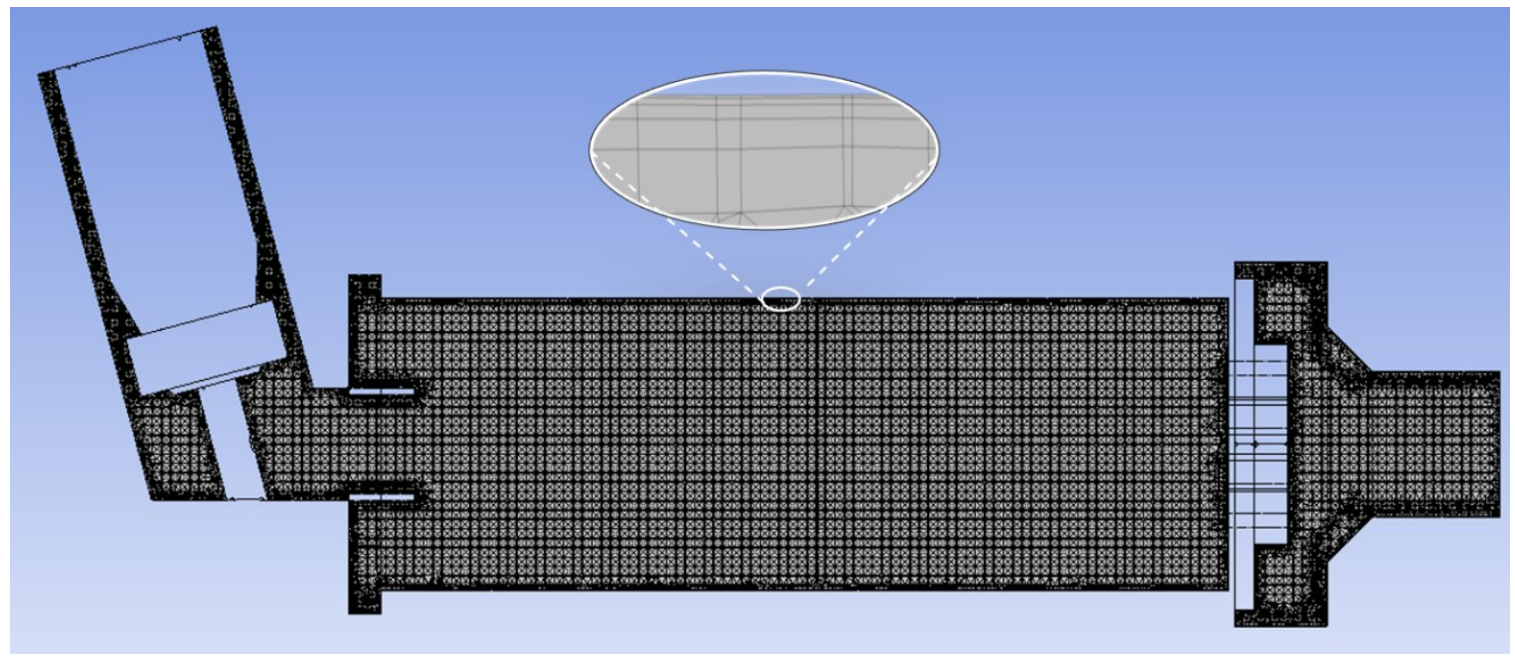

Figure 5.4: Unstructured mesh of OptiChamber ${ }^{\circledR}$ spacer geometry (6 million elements with prism layers)

\subsubsection{Convergence Studies}

A grid convergence study was conducted to ensure that a suitable number of elements were used to generate the mesh. This convergence was studied by observing 
the airflow velocity through the centerline of the spacer at various times throughout a steady state simulation at $30 \mathrm{~L} / \mathrm{min}$. Velocities were analyzed over different mesh sizes and when the variations of the velocity became independent of the mesh size (i.e. changing the mesh size did not cause variations in velocity), the grid was considered to be converged. This convergence analysis is demonstrated in Figure 5.5 and 5.6 for the Volumatic ${ }^{T M}$ and OptiChamber ${ }^{\circledR}$ spacer, respectively.

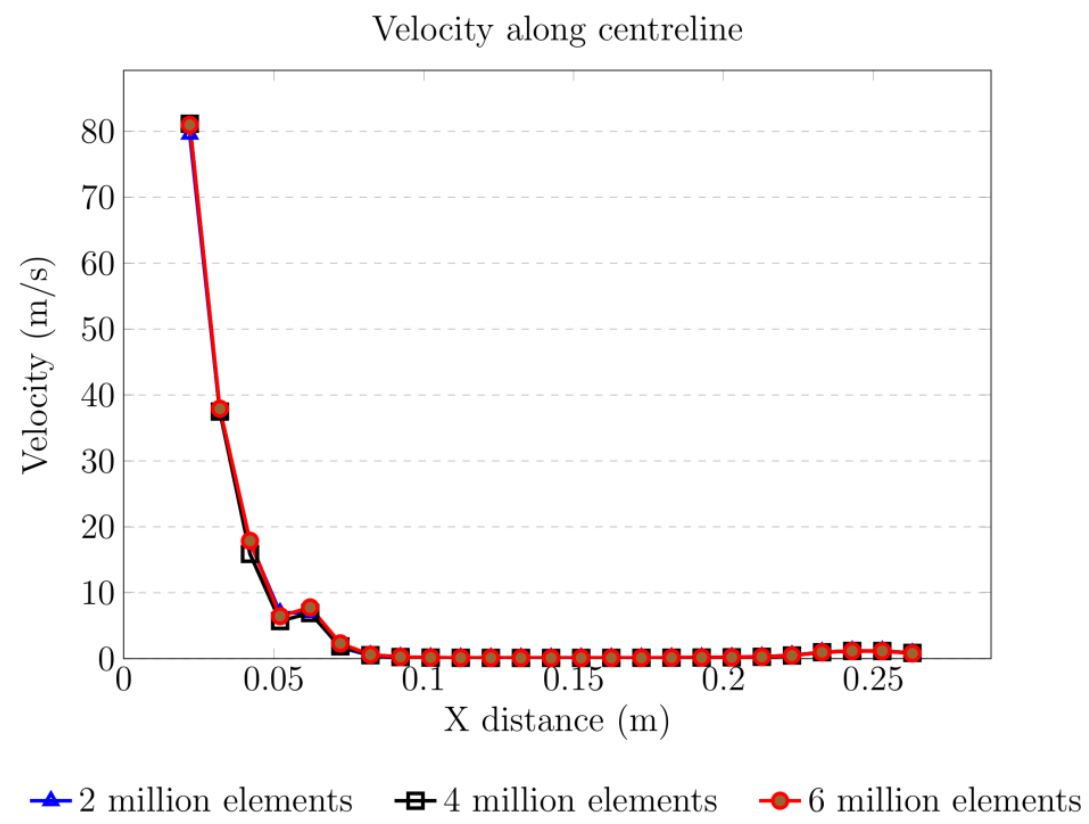

Figure 5.5: Velocity along centreline for Volumatic ${ }^{\mathrm{TM}}$ spacer 


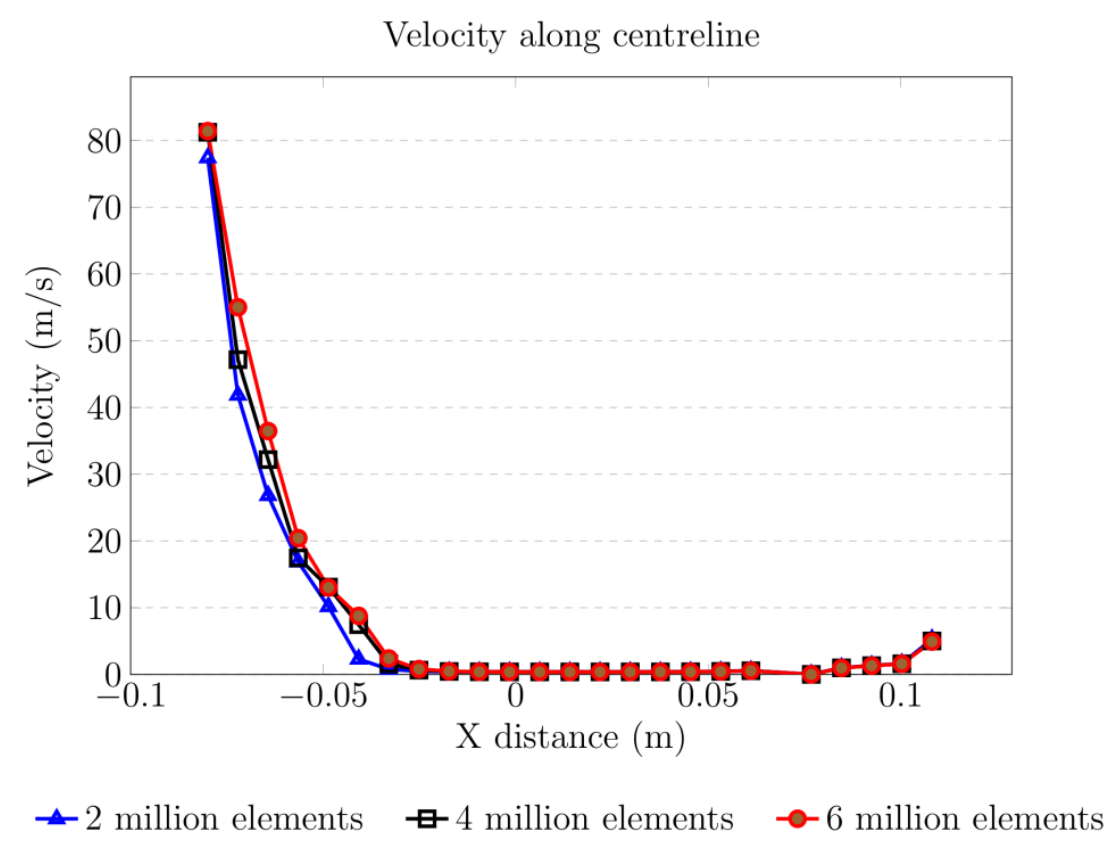

Figure 5.6: Velocity along centreline for OptiChamber ${ }^{\circledR}$ spacer

As demonstrated, once the grid was resolved past four million elements, there was a minimal change in the centreline velocity for both spacers. Additionally, results for a grid size of four million elements for both the Volumatic ${ }^{\top M}$ and OptiChamber ${ }^{\circledR}$ spacer seemed to provide similar results to a higher resolution six million elements mesh over the course of the simulation. As such, it was determined that a four million element mesh was able to provide a suitable resolution without requiring too much computational power and time.

In addition to grid size, it was also necessary to ensure time steps were small enough to capture sufficient accuracy for the simulations. A large number of elements and small time steps would require more computational power so it was important to determine the optimal to ensure sufficient accuracy is provided with minimal computational power. Temporal analysis found that a suitable time step will achieve a 
Courant number of around one at each iteration. The Courant number can be calculated as follows,

$$
C=\frac{u \cdot \Delta t}{\Delta x}
$$

Where $C$ is the Courant number, $u$ is the magnitude of the velocity $(m / s), \Delta t$ is the time step (s), and $\Delta x$ is the length interval $(m)$.

This Courant-Friedrichs-Lewy (CFL) condition was satisfied by using an adaptive time stepping procedure where time steps were adjusted to ensure the RMS Courant number remained at a value of one for the entire simulation. Using this method, the time steps ranged from around 0.1 to $0.5 \mathrm{~ms}$.

After the time step was optimized, it was then determined how long the simulation had to run. Total simulations run times were determined based on the number of particles deposited on the walls of the spacer and those which exited the domain after a specific amount of time. For an appropriate run time to be achieved, it was determined that at least $75 \%$ of the particles had to either deposit on the walls of the spacer or exit the domain. This parameter was selected to minimize the affect of computational time on numerical results. Deposition values were expected to become much less if this parameter was reduced and the run time was shortened, as the majority of the particulate would remain entrained in the flow with only a fraction depositing on the spacer surfaces. For the Volumatic ${ }^{T M}$ spacer, to satisfy this parameter, a total run time of four seconds was required. Alternatively, for the OptiChamber ${ }^{\circledR}$ 
spacer, a total run time of two seconds was required. This run time varied between the two spacers due to the volumes of the spacers themselves. A larger volume spacer (i.e. the Volumatic ${ }^{\mathrm{TM}}$ spacer) would require a longer run time as the particles had a larger distance to travel.

\subsection{Boundary Conditions}

Steady state and transient simulations were performed for each flow rate and spacer device. Steady state simulations were used to initialize the transient simulations to ensure better convergence and stability. These steady state simulations used a nozzle outlet velocity of $80 \mathrm{~m} / \mathrm{s}$ with a fixed downwards spray angle of $-6^{\circ}$ (the inlet was initialized with a jet of zero velocity) and a mass flow outlet set to the inhalation flow rate (in this case, $30 \mathrm{~L} / \mathrm{min}$ ). For transient simulations, a simplified inlet condition was produced to allow for the pMDI spray to deflect downward to $-6^{\circ}$ and back to $0^{\circ}$ to the spray axis during the actuation as reported by Crosland et al. [69]. The inlet was placed downstream of the actual nozzle, adjacent to the actuator cylindrical stem. The diameter was increased to $1.49 \mathrm{~mm}$ from the actual nozzle diameter of $0.25 \mathrm{~mm}$ to allow for more accurate meshing of the nozzle inlet. The nozzle inlet angle (in degrees) can be described by Equation (5.2) [63]. Additionally, the development of this spray angle can be seen in Figure 5.7.

$$
\propto=-\frac{1490.5}{t^{1.75}}\left[1-\exp \left(-0.000727 t^{2.5}\right)\right]
$$




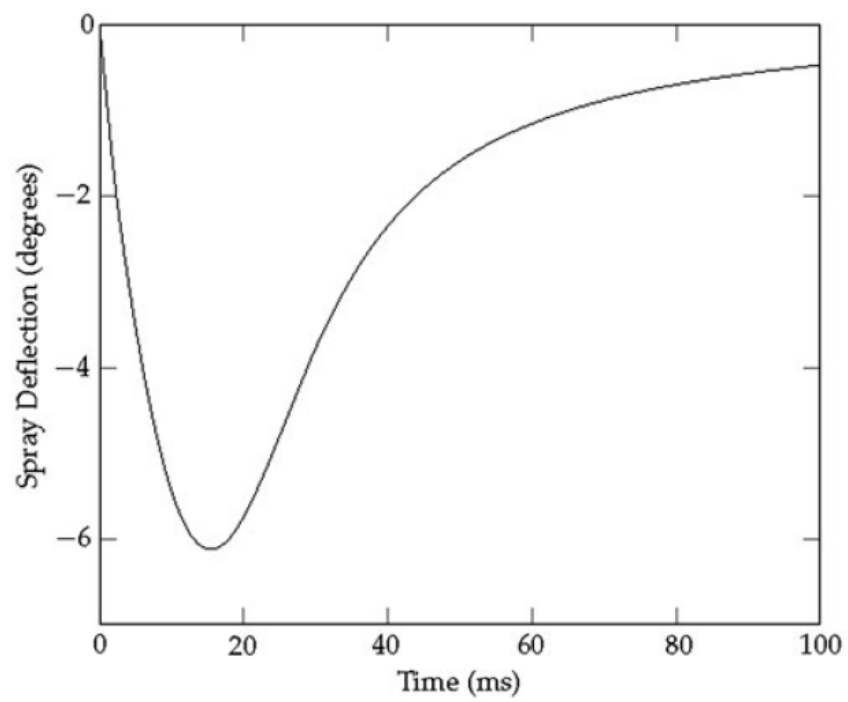

Figure 5.7: Inlet spray angle as a function of time

Further to the spray angle, a secondary opening inlet was used to simulate inflow and backflow along the sides of the pMDI. A mass flow outlet was used to simulate the required flow rate being drawn through the pMDI at the end of the spacer device. Additionally, no-slip walls with a restitution coefficient of zero were used for all surfaces in the spacers and pMDI. This ensured that any particle encountering a wall would stick to its surface without deflection. In reality, a fraction of the particles would be expected to deflect and re-entrain into the flow after impacting a solid boundary. However, due to the lack of literature on the restitution coefficient of salbutamol sulphate, it was difficult to model this deflection and re-entrainment. As such, the particles were assumed to deposit on contact with a solid surface. The boundary conditions are illustrated in Figure 5.8 and 5.9 for the Volumatic ${ }^{\mathrm{TM}}$ and OptiChamber ${ }^{\circledast}$, respectively, and listed in Table 5.1. Additionally, the fluid domain settings are listed in Table 5.2. 


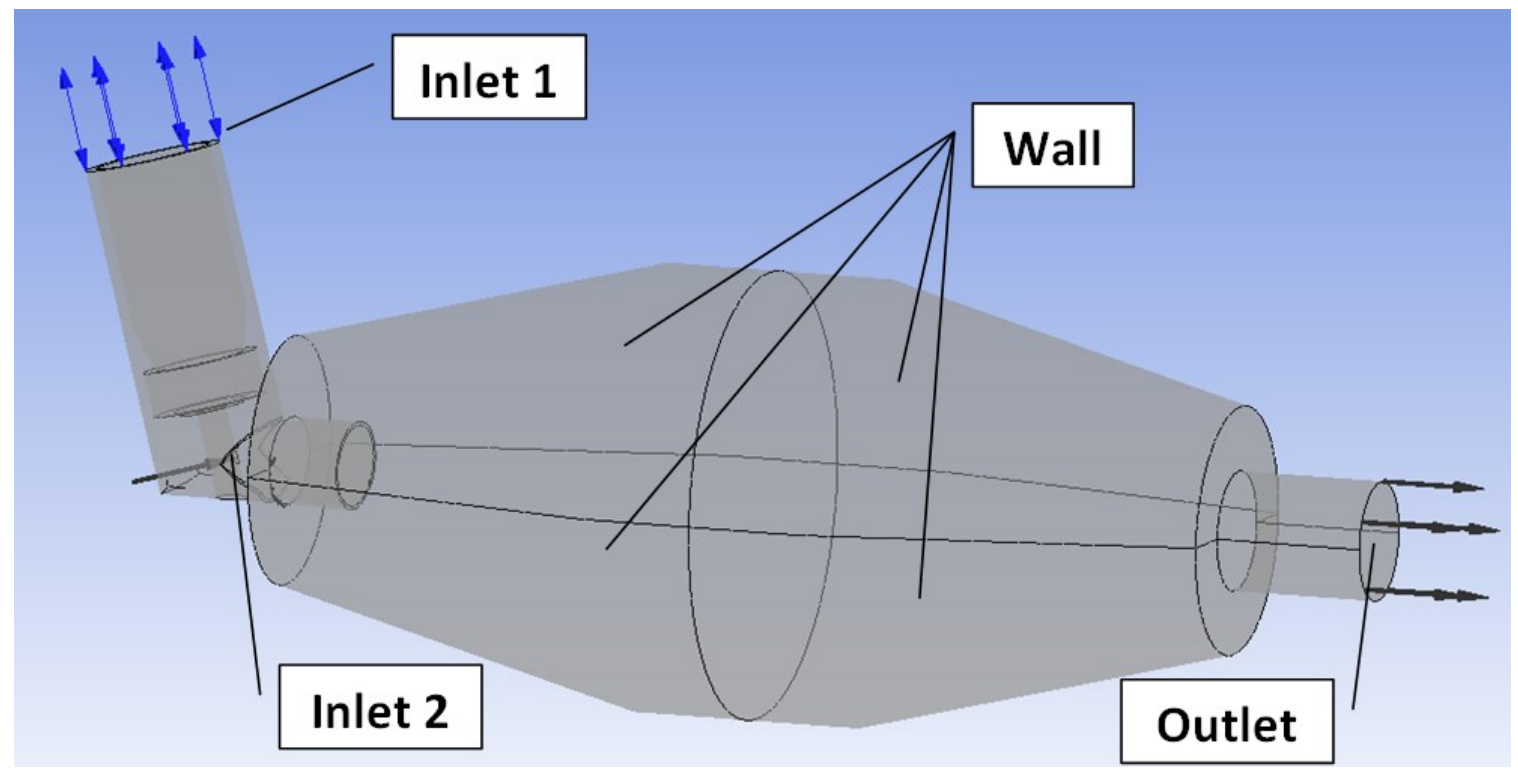

Figure 5.8: Implemented boundary conditions for the Volumatic ${ }^{\mathrm{TM}}$ spacer

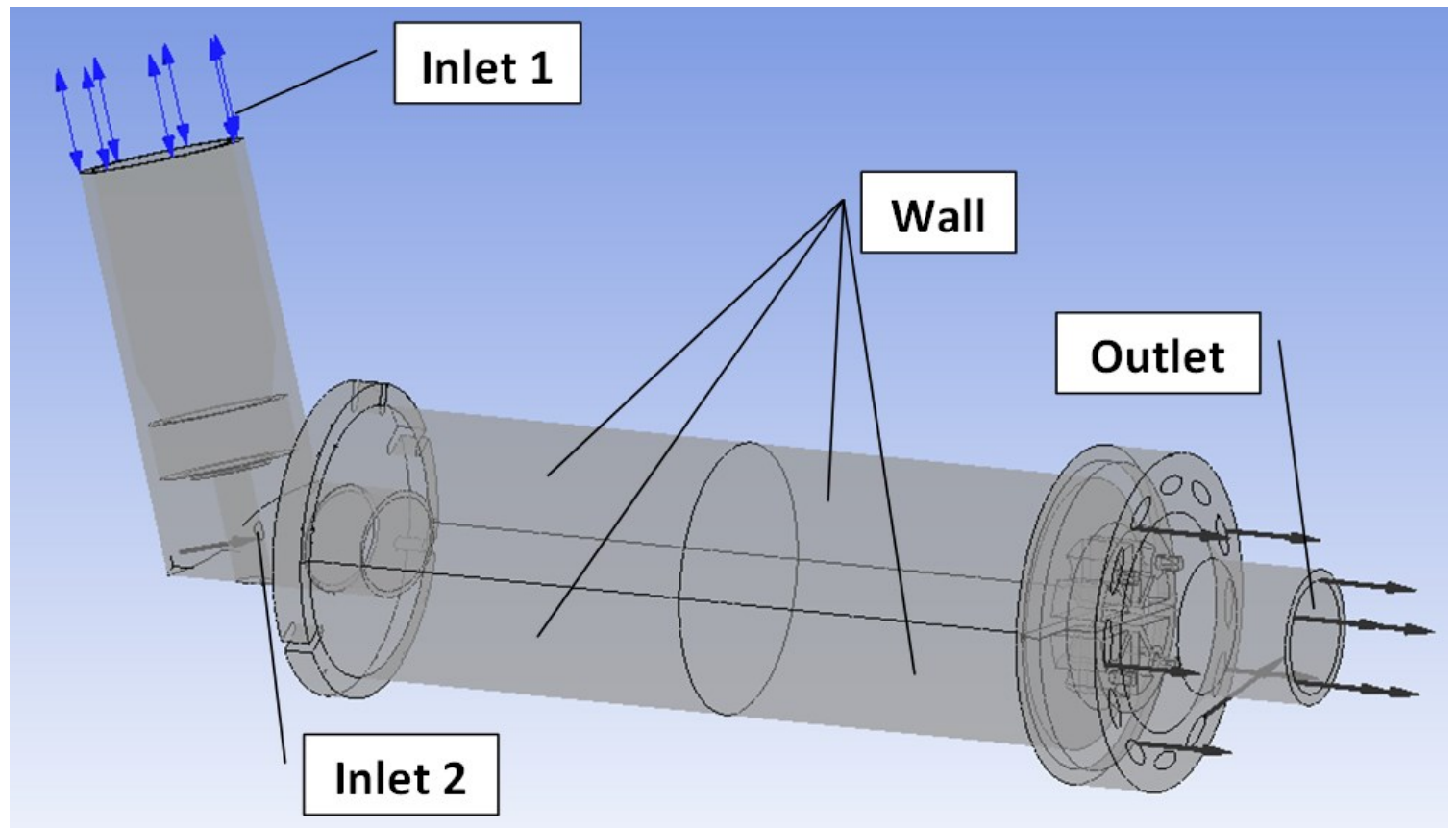

Figure 5.9: Implemented boundary conditions for the OptiChamber ${ }^{\circledast}$ spacer 
Table 5.1: Boundary conditions used for simulations

\begin{tabular}{ccc}
\hline Boundary Surface & Boundary Condition & Description \\
\hline Inlet 1 & Opening & Opening with a relative pressure of zero Pascals \\
Inlet 2 & Velocity & pMDI spray velocity incorporating $-6^{\circ}$ deflection \\
Wall & No Slip & Smooth wall with a restitution coefficient of zero \\
Outlet & Mass Flow Rate & Mass flow corresponding to inhalation rate \\
\hline
\end{tabular}

Table 5.2: Fluid domain settings used for simulations

\begin{tabular}{cc}
\hline Model & Option \\
\hline Particle Coupling & One-way Coupling \\
Drag Force Model & Schillar Naumann \\
Eddy Viscosity Ratio & $\begin{array}{c}\text { Ratio set to a value of five for } \\
\text { turbulent tracking simulations }\end{array}$ \\
Turbulence Model & Shear Stress Transport \\
\hline
\end{tabular}

\subsection{Particle Tracking}

Multiphase flow was modeled to provide an accurate simulation of the behaviour of salbutamol sulphate in the spacer devices. First, the number of particles injected into the domain had to be determined. Transient simulations using the Volumatic ${ }^{\mathrm{TM}}$ spacer (at an inhalation flow rate of $30 \mathrm{~L} / \mathrm{min}$ ) were used to test deposition of 5,000, 10,000 and 100,000 particles in the first 110 ms (i.e. just after the pMDI spray ceases). For all these three simulations, no particles were deposited on any spacer surface. Therefore, it was determined that there would be minimal difference in deposition due to the number of particles injected. As such, to balance computational complexity and time, 10,000 particles were released into the domain over 100 milliseconds (i.e. the actuation time of the puffer) with an injection velocity of $80 \mathrm{~m} / \mathrm{s}$ 
from the nozzle inlet. For simplicity, one-way coupling was used to describe the particulate phase. Additionally, particle tracks were implemented using either mean flow or turbulent tracking to determine the method of deposition. These models are discussed further in Section 5.4.1 and 5.4.2.

A monodisperse particle size of 2.11 micron was used to describe the size distribution of the particulate. Due to the lack of functionality for ANSYS CFX to implement the Cunningham correction factor for drag on small particles (less than one micron), a particle size above one micron had to be implemented. The inclusion of smaller particles in the size distribution without the Cunningham correction factor would overestimate the drag force on the particulate which would result in an underestimate of particle deposition (due to lower mobility). The monodisperse size of 2.11 micron was determined from the median of particle size measurements of salbutamol sulphate released from the Teva-Salbutamol HFA MDI using phase doppler anemometry (PDA) performed by Alatrash [70]. It is important to note that if larger particles were included in this size distribution, the mechanism of deposition would change. Large particles (greater than five micron) would typically deposit due to inertial impaction.

After the size distribution parameters were set, salbutamol sulphate particles were specified as a particle transport fluid with a Schiller-Naumann drag model. This drag model is ideal for small-particle Reynolds numbers when fluid particles behave similar to solid particles and for flow around a single sphere. Therefore, this drag model 
is suitable as the particles of salbutamol sulphate can be idealized as spheres with a constant diameter [71].

The deposition was determined through custom FORTRAN and MATLAB code. The FORTRAN code was used to extract the final position of each of the 10,000 particles. These positions were exported to MATLAB and analysed using a script that determined if (and if so, where) the final position of the particles intersected with the geometry of the spacer or exited the domain.

\subsubsection{Mean Flow Tracking}

Mean flow tracking conducts simulations without modeling, which allows for the fluctuation component of the instantaneous fluid velocity, i.e. $u_{i}$ in Equation (3.6), to be considered zero. The implementation of mean flow tracking assumes deposition to only occur due to the average flow.

\subsubsection{Turbulent Tracking}

Turbulent tracking, also known as the random walk model, considers both the mean and fluctuating components of the instantaneous velocity in Equation (3.6). The inclusion of the fluctuating component results in particle dispersion in turbulent flow since particles can follow independent tracks at each injection point.

To model this turbulent particle dispersion, each particle is assumed to interact with eddies as it travels throughout the domain. Each eddy has a characteristic length, lifetime, and velocity. When a particle interacts with an eddy, the fluctuating 
component of the instantaneous fluid velocity is assumed to be equal to the fluctuating velocity of the eddy. This causes the fluctuating velocity to dominate. A particle ends its interaction with an eddy if the lifetime of the eddy is exceeded or the particle travels the eddy length. At this point, the particle is assumed to enter another new eddy. To implement turbulent tracking, an eddy viscosity ratio, as in Equation (3.20), was set to the default value of five. 


\section{Chapter 6: Results \& Discussion}

\subsection{Experimental Results}

Experiments were conducted to assess the regional deposition of salbutamol

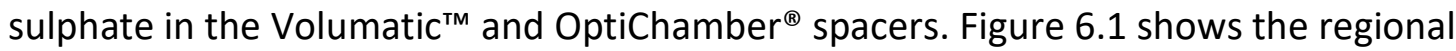
deposition of salbutamol sulphate in the spacers at inspiratory flow rates of 30,45 , and $60 \mathrm{~L} / \mathrm{min}$.

A

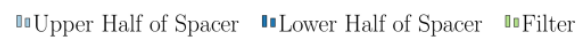

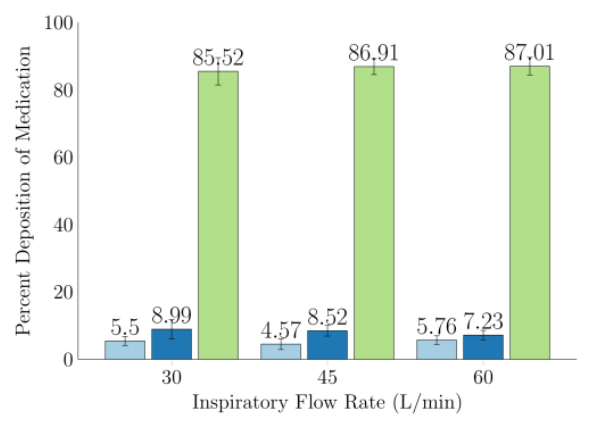

B

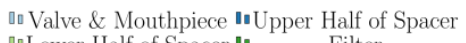
"ᄆ Lower Half of Spacer $\|$ Filter

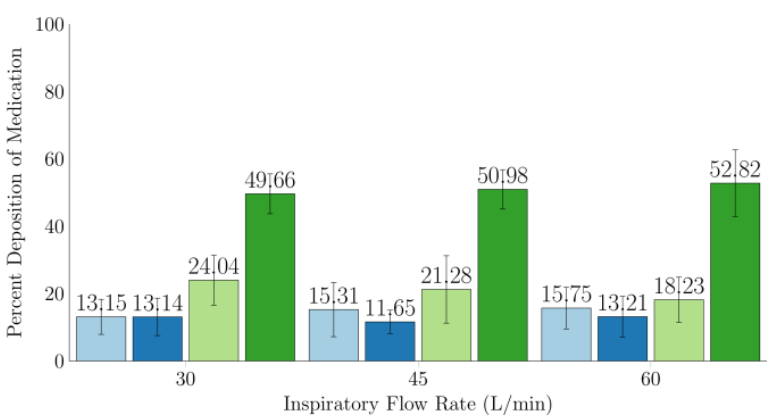

Figure 6.1: Experimental results of the percent deposition of medication in the upper and lower regions of the (a) Volumatic ${ }^{\mathrm{TM}}$ and (b) OptiChamber ${ }^{\circledR}$ spacers $^{3}$

For higher flow rates, the Volumatic ${ }^{\mathrm{TM}}$ and OptiChamber ${ }^{\circledR}$ spacers allowed a slightly greater amount of medication to reach the patient. Figure 6.1 also shows a greater amount of deposition in the lower half of both spacers for all flow rates. This non-symmetrical deposition was expected due to the downward spray angle deflection from the pMDI itself as reported by Crosland et al. [69]. Additionally, for the

\footnotetext{
${ }^{3}$ Note that the error bars demonstrated in Figures 6.1 through 6.3 demonstrate the standard deviation of the average deposition measurements
} 
OptiChamber ${ }^{\circledast}$ spacer, the valve and mouthpiece entrained a significant amount of particles for the flow rates tested (upwards of $15 \%$ ).

Figure 6.2 demonstrates the total percentage of deposition in the spacer as opposed to the filter. The amount of medication reaching the patient is indicated by the decrease in deposition in the spacer and increase in deposition in the filter for higher flow rates as illustrated by the experimental results. Comparing the Volumatic ${ }^{\mathrm{TM}}$ and OptiChamber ${ }^{\circledast}$ spacer, shows a larger amount of medication will reach the patient using the Volumatic ${ }^{\top M}$ spacer at all flow rates. Conversely, the OptiChamber ${ }^{\circledR}$ delivers on average about $40 \%$ less medication than the Volumatic ${ }^{\mathrm{TM}}$ spacer with much of the medication depositing in the spacer itself and on the valve and mouthpiece.

A

『spacer $\quad \square$ Filter

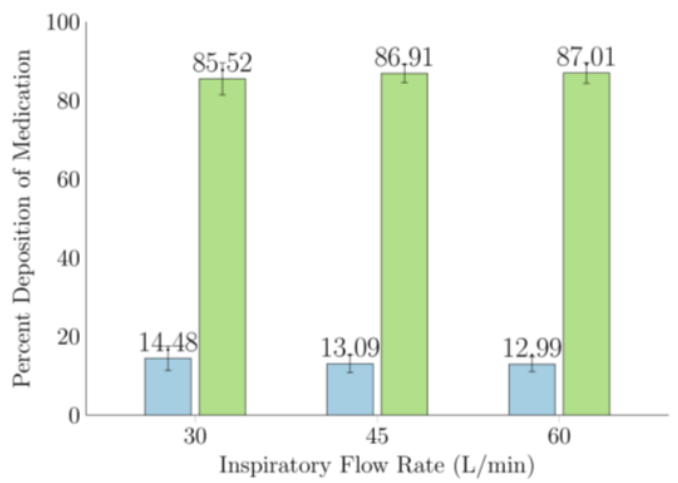

B

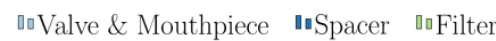

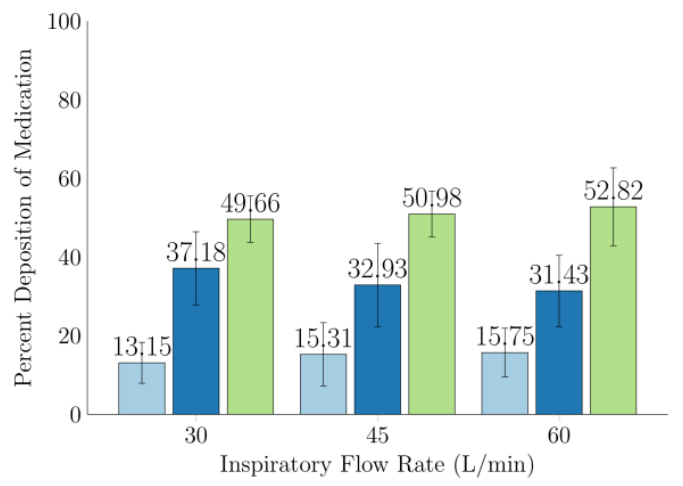

Figure 6.2: Experimental results of the percent deposition of medication in the spacer and filter of the (a) Volumatic ${ }^{\mathrm{TM}}$ and (b) OptiChamber ${ }^{\circledR}$ spacers

For all flow rates, a greater amount of deposition was also found to occur at the distal half of the spacers, as demonstrated in Figure 6.3. It should be noted that the 
distal half of the OptiChamber ${ }^{\circledast}$ spacer included deposition in the valve and mouthpiece of the spacer. For the OptiChamber ${ }^{\circledast}$, this result suggested that more medication is likely to impinge in the valve portion of the spacer before reaching the patient, significantly reducing the amount of medication delivered. These deposition patterns were also demonstrated by the Volumatic ${ }^{\mathrm{TM}}$ spacer, however, the amount of distal deposition was not so extreme. It is conjectured that more medication would reach a patient using the Volumatic $^{\mathrm{TM}}$ spacer due to the absence of a valve.

It should also be recognized that for the OptiChamber ${ }^{\circledR}$ and Volumatic ${ }^{\mathrm{TM}}$ spacer, the amount of proximal deposition is less than the amount of distal deposition. This is likely due to the high plume velocity of the pMDI aerosol spray which will propel the medication a large distance in a short amount of time.

A

घuProximal $\quad$ aDistal

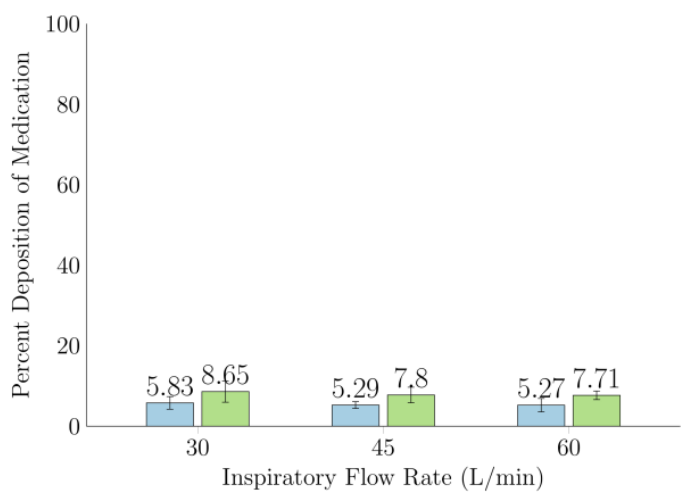

B
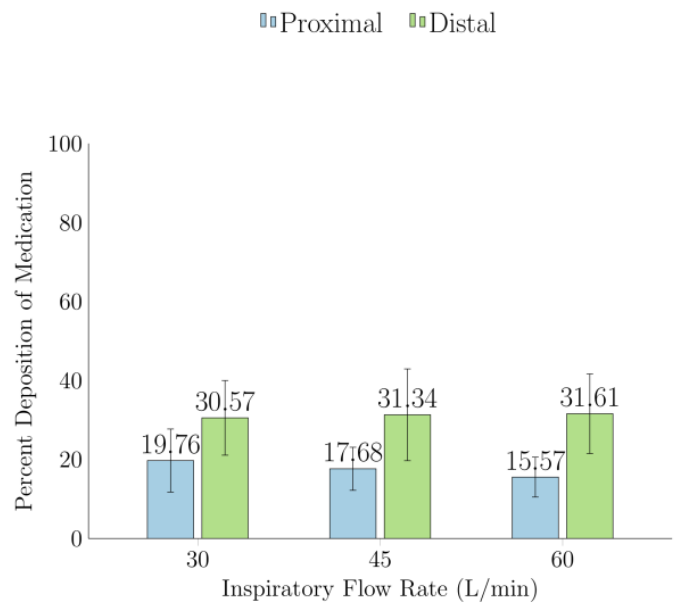

Figure 6.3: Experimental results of the percent deposition of medication in the proximal and distal of the (a) Volumatic ${ }^{\mathrm{TM}}$ and (b) OptiChamber ${ }^{\circledR}$ spacers 


\subsubsection{Correlated t-test}

Due to the large amount of variance in the experimental results for assessing distal and proximal deposition, a correlated t-test was conducted to ensure the reported

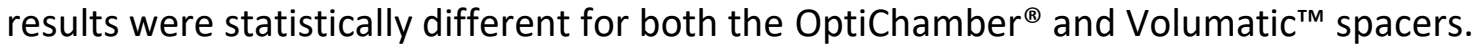
All the values used in the t-test were the deposition values of salbutamol sulphate in micrograms.

The null hypothesis for the first t-test in this analysis was that the mean difference between distal and proximal deposition for each trial is zero. A t value was calculated using Equation (6.1) as follows,

$$
t=\frac{M-\mu}{s_{M}}
$$

Where $M$ is the sample mean, $\mu$ is zero (as the null hypothesis assumes the mean

difference between the distal and proximal deposition for each trail is zero) and $S_{M}$ is the standard error of the mean.

Similarly, a second t-test was conducted to ensure that the differences between the lower and upper deposition were statistically different. The null hypothesis for this second t-test was that the mean difference between lower and upper deposition for each trial is zero. Tables 6.1 and 6.2 list the $t$ values and corresponding two-tailed probability values (found using appropriate z-tables) for each flow rate and spacer. Using an alpha of 0.05 , indicating a 95\% confidence interval, each computed mean is significantly different from zero. Therefore, the lower deposition found from 
this experimental analysis can be said to be statistically higher than the upper deposition. In addition, the distal deposition can be said to be statistically higher than the proximal deposition.

Table 6.1: Calculated $t$ values and two-tailed probability to prove statistical difference between the experimental deposition found on the lower versus upper regions and distal versus proximal regions of the Volumatic ${ }^{\mathrm{TM}}$ spacer for each corresponding flow rate

\begin{tabular}{|c|c|c|c|c|c|c|}
\hline \multicolumn{7}{|c|}{ Volumatic Spacer } \\
\hline \multirow[b]{2}{*}{$\begin{array}{c}\text { Flow rate } \\
(\mathrm{L} / \mathrm{min})\end{array}$} & \multicolumn{2}{|c|}{$\underline{\text { Standard error of mean }\left(s_{M}\right)}$} & \multicolumn{2}{|c|}{$\underline{\mathrm{t}-\text { Value }}$} & \multicolumn{2}{|c|}{ Two-tailed probability $(\mathrm{p})$} \\
\hline & $\begin{array}{l}\text { Lower-Upper } \\
\text { Difference }\end{array}$ & $\begin{array}{l}\text { Distal-Proximal } \\
\text { Difference }\end{array}$ & $\begin{array}{l}\text { Lower-Upper } \\
\text { Difference }\end{array}$ & $\begin{array}{l}\text { Distal-Proximal } \\
\text { Difference }\end{array}$ & $\begin{array}{l}\text { Lower-Upper } \\
\text { Difference }\end{array}$ & $\begin{array}{l}\text { Distal-Proximal } \\
\text { Difference }\end{array}$ \\
\hline 30 & 0.57 & 0.53 & 6.08 & 5.32 & 0.00030 & 0.00071 \\
\hline 45 & 0.72 & 0.61 & 5.52 & 4.13 & 0.00056 & 0.0033 \\
\hline 60 & 0.12 & 0.31 & 12.18 & 7.89 & $<0.000010$ & 0.000048 \\
\hline
\end{tabular}

Table 6.2: Calculated $t$ values and two-tailed probability to prove statistical difference between the experimental deposition found on the lower versus upper regions and distal versus proximal regions of the OptiChamber ${ }^{\circledast}$ spacer for each corresponding flow rate

\begin{tabular}{|c|c|c|c|c|c|c|}
\hline \multicolumn{7}{|c|}{ OptiChamber Spacer } \\
\hline \multirow[b]{2}{*}{$\begin{array}{c}\text { Flow rate } \\
(\mathrm{L} / \mathrm{min})\end{array}$} & \multicolumn{2}{|c|}{ Standard error of mean $\left(s_{M}\right)$} & \multicolumn{2}{|c|}{$\underline{\mathrm{t}-\text { Value }}$} & \multicolumn{2}{|c|}{ Two-tailed probability $(\mathrm{p})$} \\
\hline & $\begin{array}{l}\text { Lower-Upper } \\
\text { Difference }\end{array}$ & $\begin{array}{c}\text { Distal-Proximal } \\
\text { Difference }\end{array}$ & $\begin{array}{l}\text { Lower-Upper } \\
\text { Difference }\end{array}$ & $\begin{array}{c}\text { Distal-Proximal } \\
\text { Difference }\end{array}$ & $\begin{array}{l}\text { Lower-Upper } \\
\text { Difference }\end{array}$ & $\begin{array}{l}\text { Distal-Proximal } \\
\text { Difference }\end{array}$ \\
\hline 30 & 2.39 & 2.87 & 4.57 & 3.77 & 0.00044 & 0.0021 \\
\hline 45 & 2.09 & 2.35 & 4.23 & 5.81 & 0.00085 & 0.000045 \\
\hline 60 & 2.16 & 3.98 & 2.33 & 4.03 & 0.035 & 0.0012 \\
\hline
\end{tabular}

\subsection{Numerical Results}

For the numerical analysis, transient simulations were conducted to study the behaviour of salbutamol sulphate in the Volumatic ${ }^{\top \mathrm{M}}$ and OptiChamber ${ }^{\circledR}$ spacers. $^{2}$ 
Simulations incorporating the downward spray angle developed over the actuation of the pMDI were carried out at an inhalation flow rate of $30 \mathrm{~L} / \mathrm{min}$. Using URANS and particle tracking, it was possible to find where particles deposited within the spacer.

The 10,000 particles released into the domain were tracked over the duration of the simulation, four seconds for the Volumatic ${ }^{T M}$ spacer and two seconds for the OptiChamber ${ }^{\circledast}$ spacer. These simulations were run on a computational cluster, using 36 partitions and cores with $72 \mathrm{~GB}$ of RAM. On this cluster, simulations for the Volumatic ${ }^{\mathrm{TM}}$ spacer took about four days to complete, while simulations for the OptiChamber ${ }^{\circledR}$ spacer took about seven days.

The particle paths were tracked using mean flow and turbulent tracking to locate the point at which the medication would deposit. The resolved particle tracks for both tracking methods are illustrated in Figure 6.4. It is important to note that it is possible that not all 10,000 particles will deposit on the spacer surfaces or leave the domain (i.e. a small amount will remain entrained in the flow). However, run times were configured to ensure at least $75 \%$ of the particles either deposited on the spacer surfaces or left the domain. The following results exclude the small percentage of particles which remained entrained in the flow at the end of the simulation. 

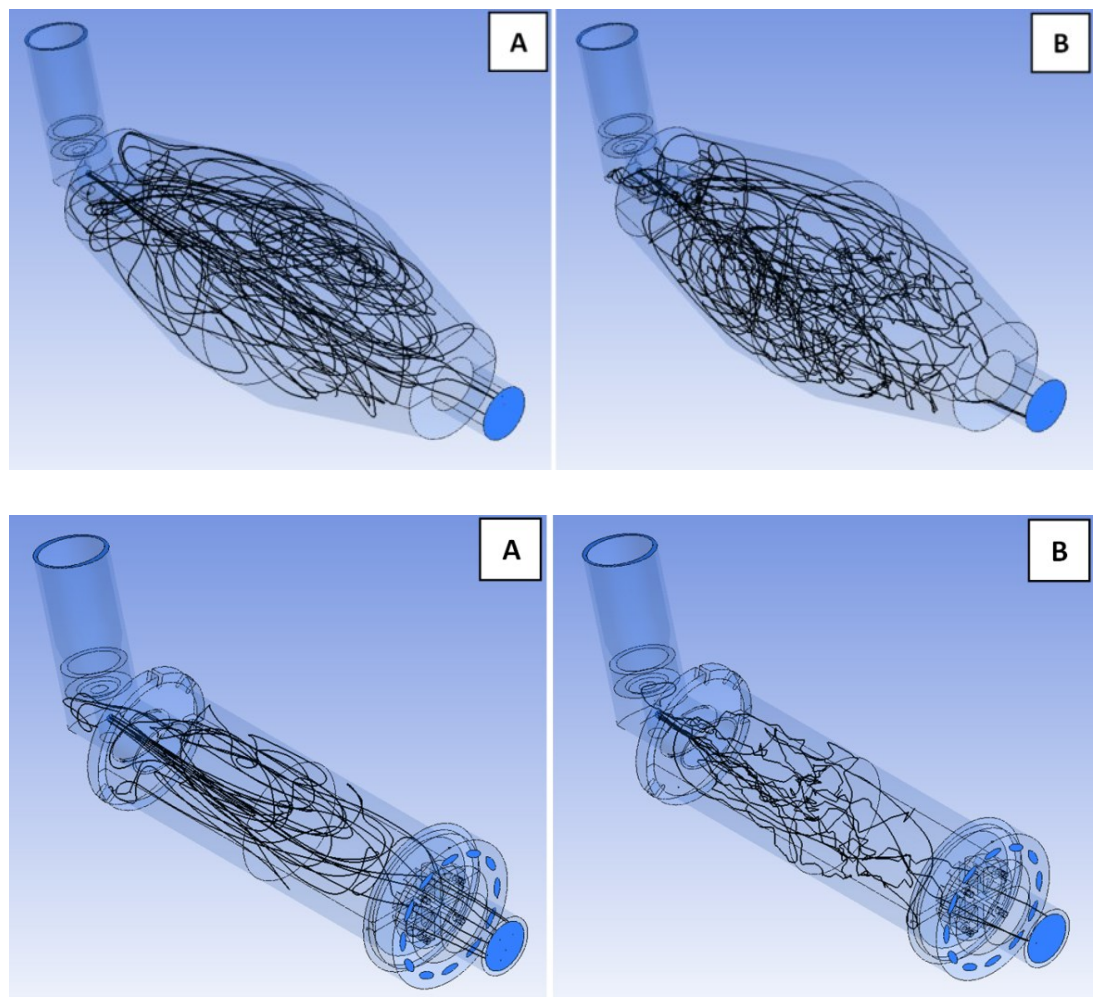

Figure 6.4: Mean flow (A) and turbulent (B) particle tracks for the Volumatic ${ }^{\mathrm{TM}}$ (top) and OptiChamber ${ }^{\circledR}$ (bottom) spacer

Firstly, mean flow tracking was used to track the movement of the particles under the average flow without a fluctuating component of velocity. In this type of tracking, the deposition mechanism which was most dominant was inertial impaction. Since only the average flow is considered without fluctuations, particles are entrained in a steady flow with low turbulence and are traveling at a high velocity. Any sudden changes in the flow direction will cause the particles to deviate from the air streamlines and maintain their existing trajectory due to their momentum. As a result, these particles will impact the nearest wall due to their large inertia. A large percent penetration of particles leaving the domain was expected due to minimal turbulent effects. Figure 6.5 and 6.6 demonstrate the numerical particle deposition in both 
spacers using mean-flow tracking. With the implementation of mean flow tracking, numerical results seemed to over predict experimental results for both the Volumatic ${ }^{\mathrm{TM}}$ and OptiChamber ${ }^{\circledR}$ spacer. The majority of particles traveled the length of the spacers with minimal deposition which resulted in a much larger penetration (i.e. the amount of medication reaching the patient) than experimental results.

A 『u Upper Half of Spacer

॥ Lower Half of Spacer $\llbracket$ Filter

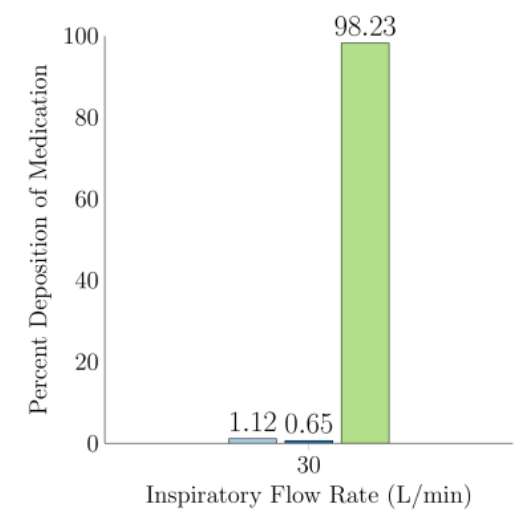

B

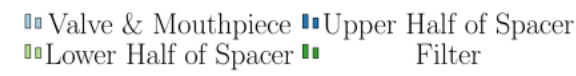

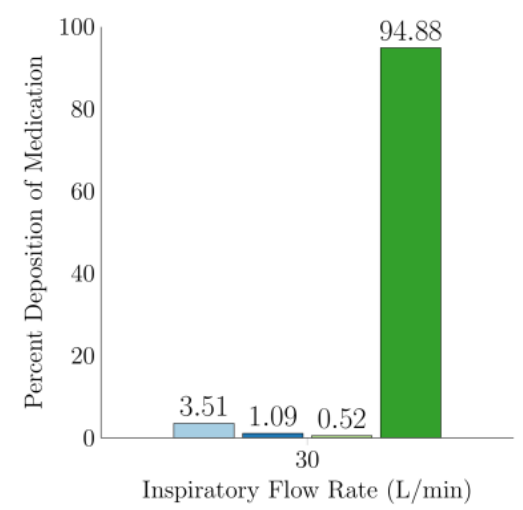

Figure 6.5: Numerical results of the percent deposition of medication in the upper and lower regions of the (a) Volumatic ${ }^{\mathrm{TM}}$ and (b) OptiChamber ${ }^{\circledR}$ spacers using mean-flow tracking

A

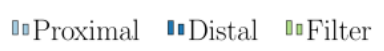

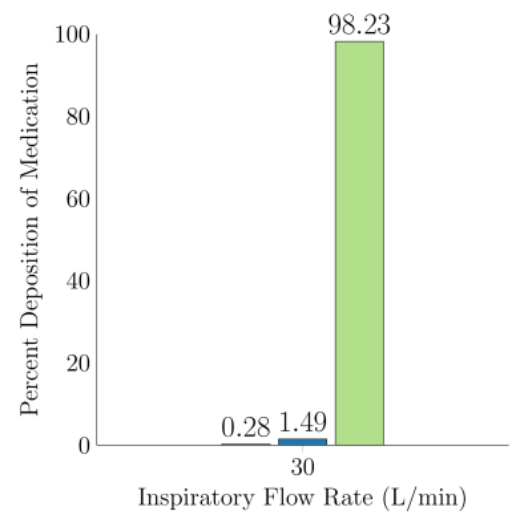

B पnValve \& Mouthpiece " Proximal an Distal In Filter

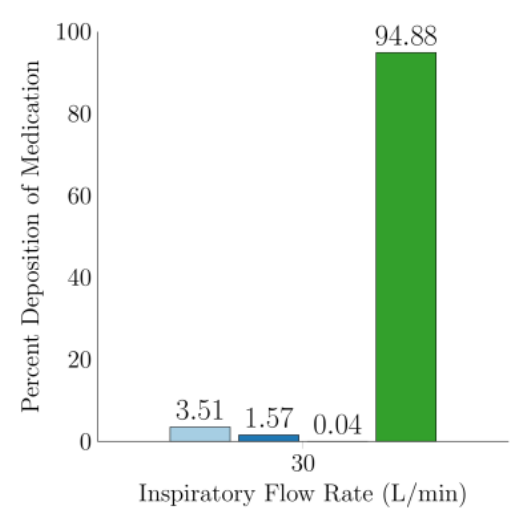

Figure 6.6: Numerical results of the percent deposition of medication in the proximal and distal regions of the (a) Volumatic ${ }^{T M}$ and (b) OptiChamber ${ }^{\circledR}$ spacers using mean-flow tracking 
Conversely, turbulent tracking considers the interaction of particles with eddies.

This eddy interaction causes a large fluctuating component of velocity which dominates over the mean velocity. This causes particle dispersion in a turbulent flow with the possibility of each particle following independent tracks. As such, particles tracked using this method are more likely to deposit due to inherent turbulence in the flow. Figure 6.7 and 6.8 demonstrate the numerical particle deposition in both spacers using turbulent tracking. With the implementation of turbulent tracking, numerical results seemed to reflect experimental results much better for both the Volumatic ${ }^{\mathrm{TM}}$ and OptiChamber ${ }^{\circledR}$ spacer. The penetration (i.e. the amount of medication reaching the filter) varied from experimental results by a relative error of about $12 \%$ for the Volumatic ${ }^{\mathrm{TM}}$ spacer and $19 \%$ for the OptiChamber ${ }^{\circledR}$ spacer. Furthermore, these results seem to better demonstrate the larger deposition of medication on the lower surface of the spacers (as opposed to the upper surface) due to the downwards spray angle of the pMDI.

A

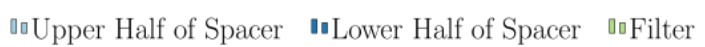

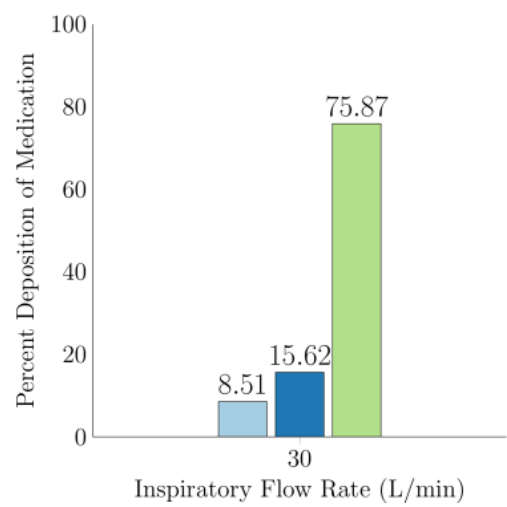

B

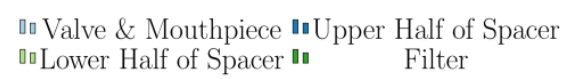

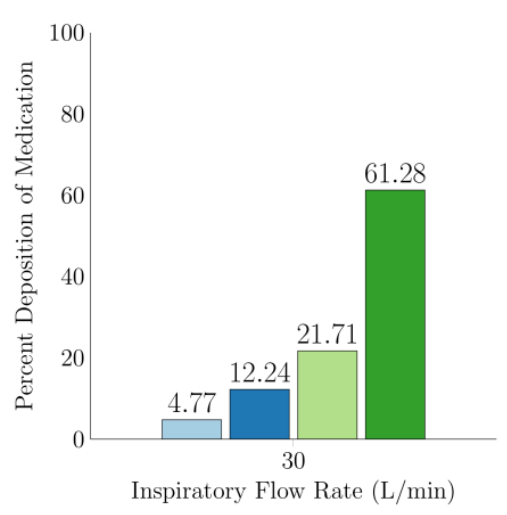

Figure 6.7: Numerical results of the percent deposition of medication in the upper and lower regions of

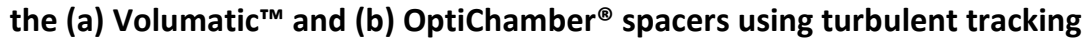


A

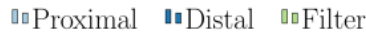

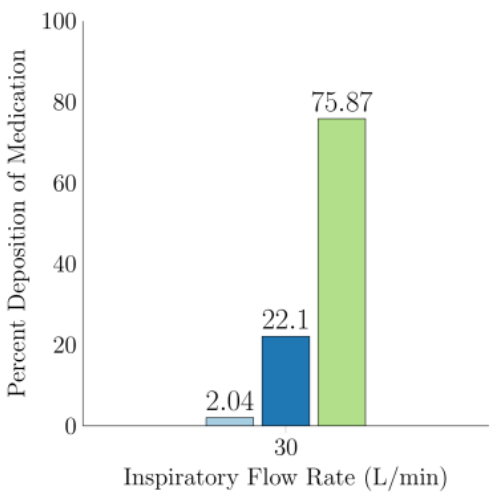

B

पaValve \& Mouthpiece IロProximal Distal u Filter

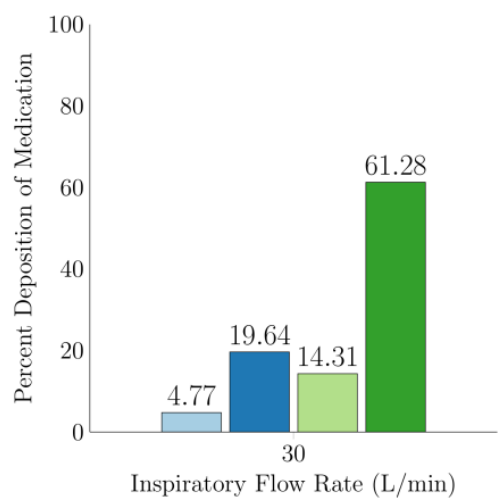

Figure 6.8: Numerical results of the percent deposition of medication in the proximal and distal regions of the (a) Volumatic ${ }^{\mathrm{TM}}$ and (b) OptiChamber ${ }^{\circledR}$ spacers using turbulent tracking

For both spacers, to observe deposition mechanisms which may be a result of spacer design, regions of turbulence kinetic energy (TKE) were quantified. Regions of high TKE can indicate the presence of turbulence eddies such as at regions of flow separation. These regions will cause small diameter particle deposition as their particle tracks can be altered by the turbulence. If these regions are consistently found at separation points, design changes can be made to minimize the creation of these eddies. As such, Figure 6.9 and 6.10 demonstrate regions of TKE for both the URANS simulation of the Volumatic ${ }^{\mathrm{TM}}$ and OptiChamber ${ }^{\circledast}$, respectively, at a flow rate of 30 $\mathrm{L} / \mathrm{min}$ over the first $110 \mathrm{~ms}$ of the run.

As illustrated, regions of high turbulence appear at the inlet of both spacers consistently over the actuation of the spray. This turbulence appears to dissipate as the length of the spacer is traversed. In the Volumatic ${ }^{\mathrm{TM}}$ spacer, a region of turbulence appears to be confined in the first half of the lower proximal quadrants, far from the 
midplane of the spacer. It is conjectured that this will result in more deposition in the bottom proximal section of the spacer. Alternatively, in the OptiChamber ${ }^{\circledR}$ spacer, a region of turbulence appears across a large section of the lower proximal quadrants, just barely passing the midplane of the spacer into the distal sections. Furthermore, between a time of 0.06 and 0.1 seconds, this region of turbulence reaches a portion of the upper proximal quadrants. This could result in more deposition in the upper proximal section of the spacer despite the downwards spray angle of the pMDI.

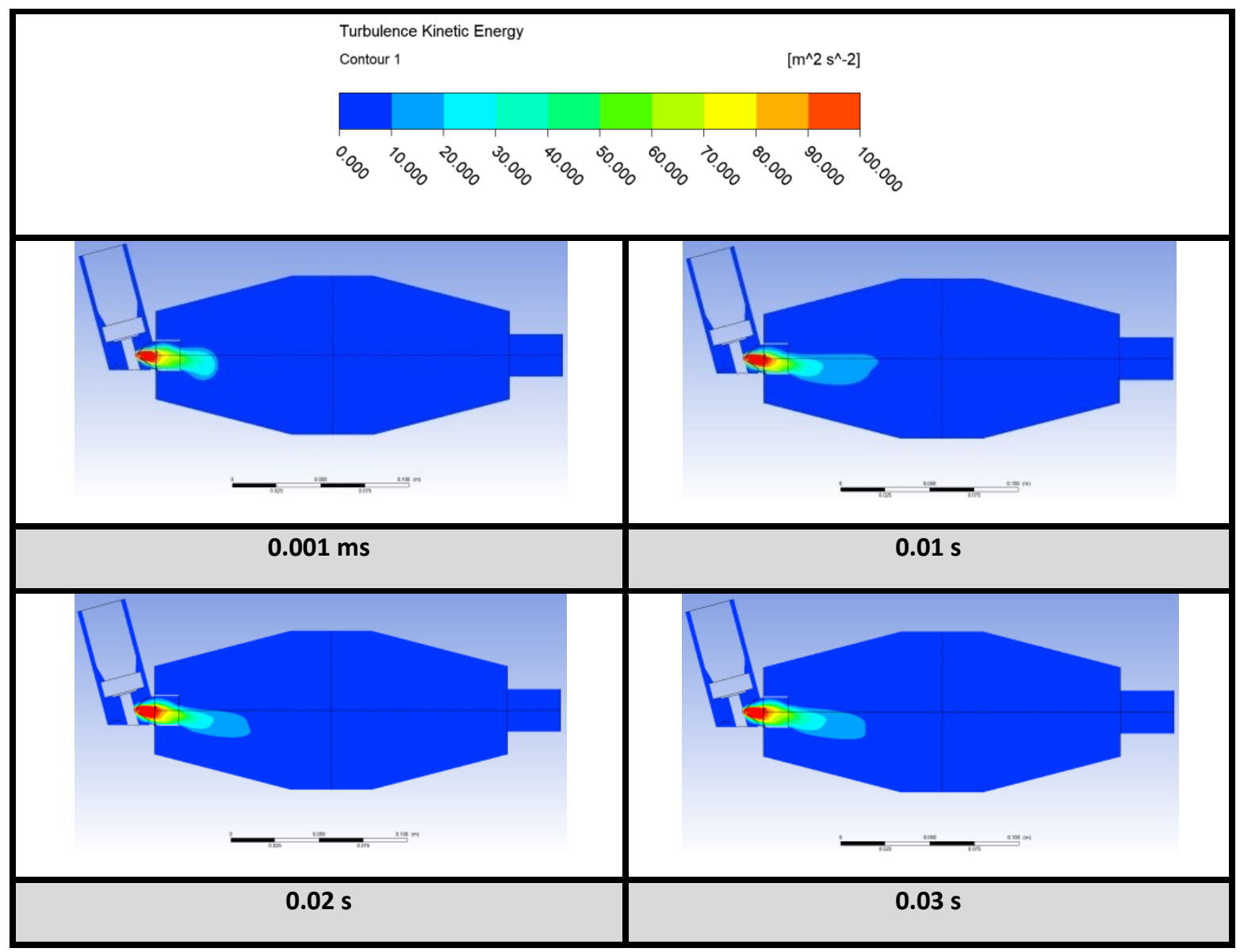




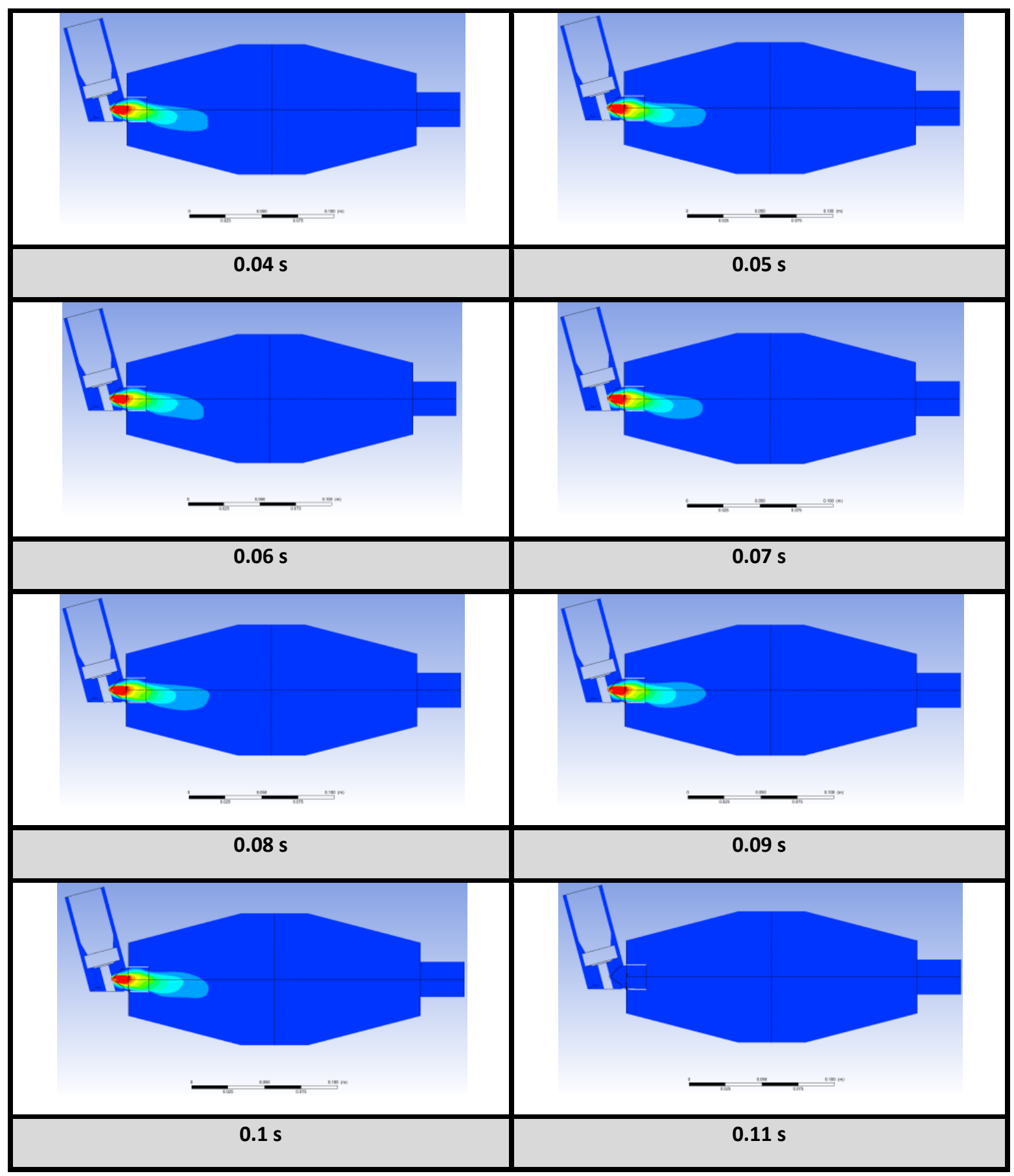

Figure 6.9: Numerical results of the turbulence kinetic energy in the Volumatic ${ }^{\mathrm{m}} \mathrm{spacer}$ at $30 \mathrm{~L} / \mathrm{min}$ 


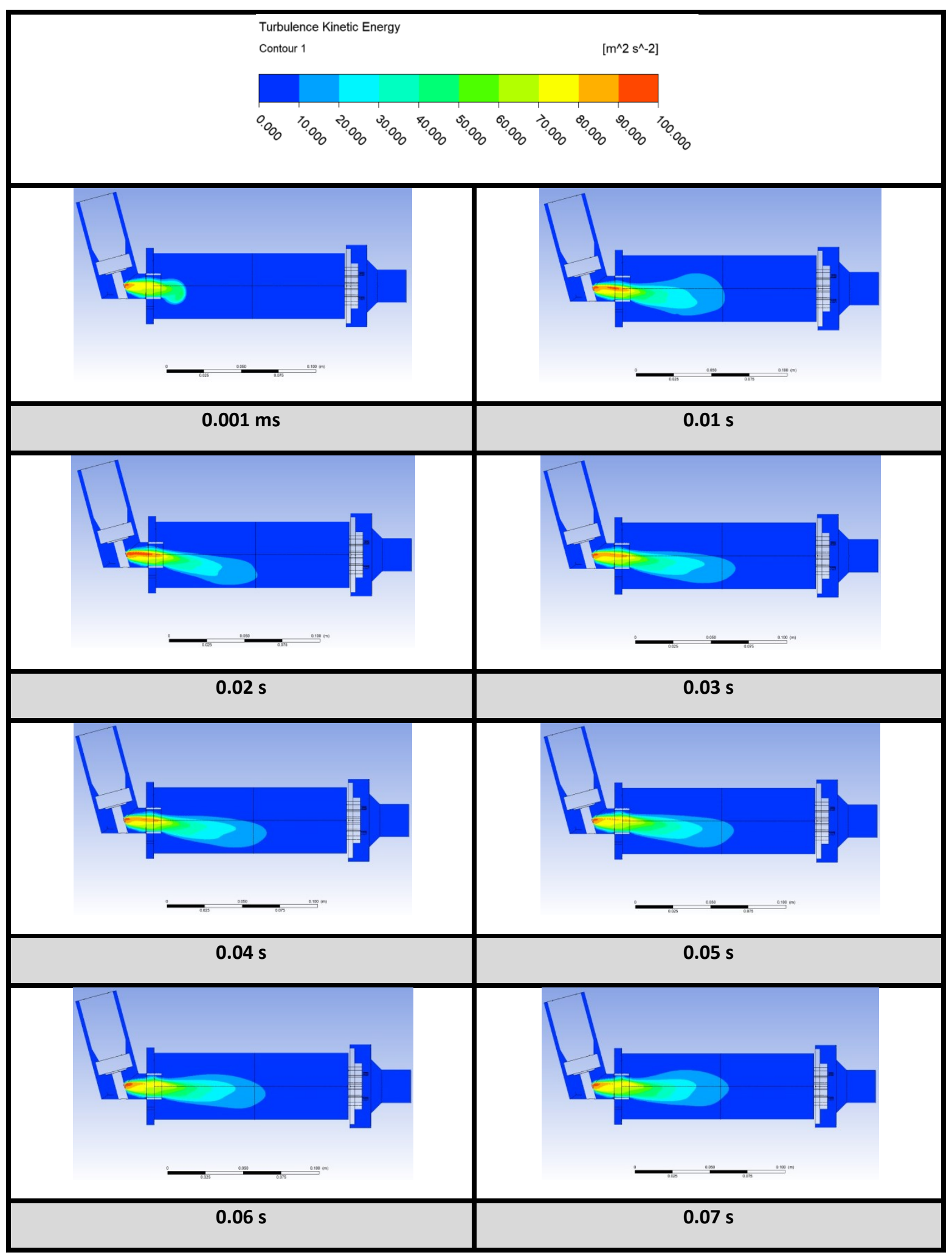




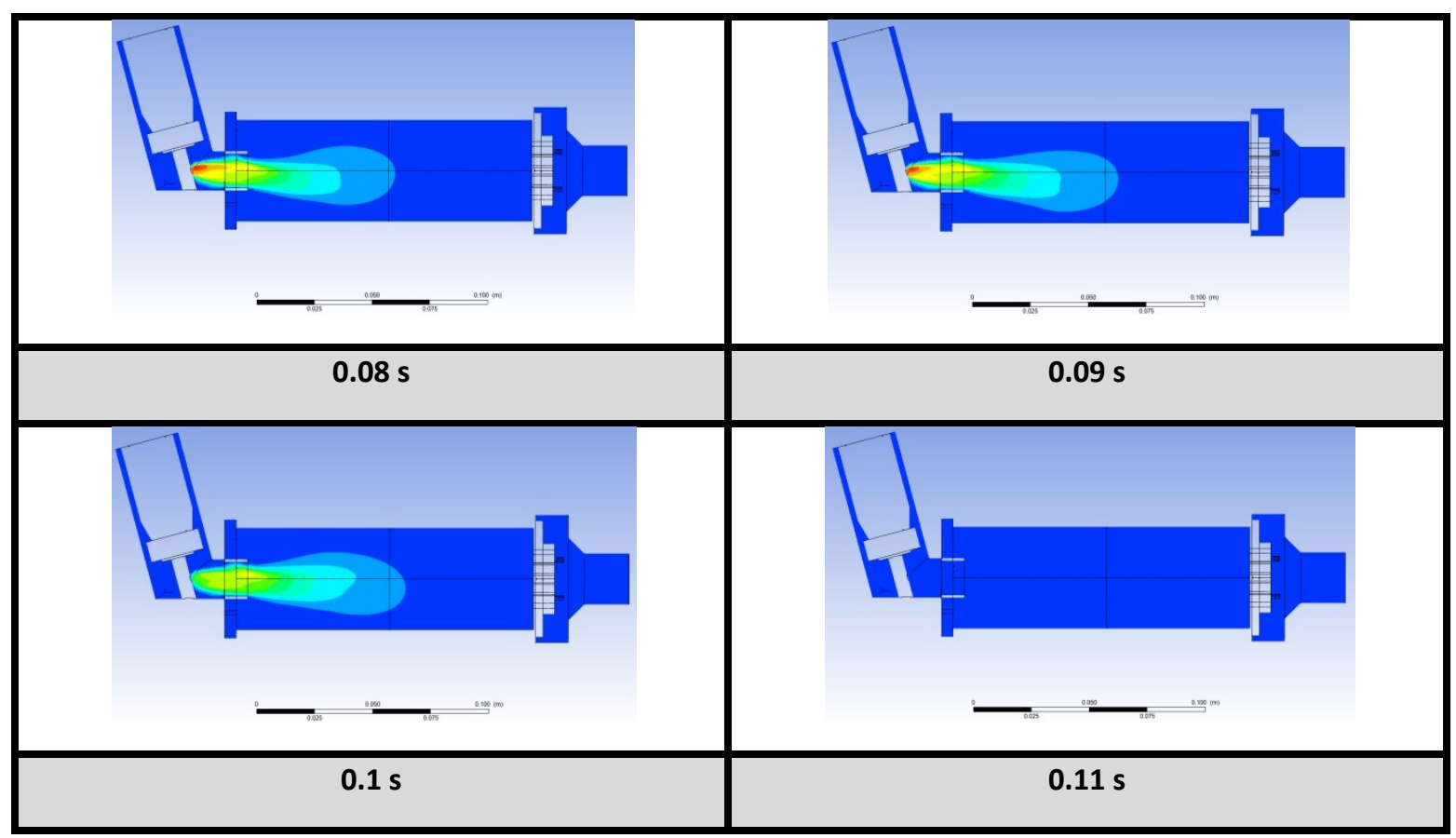

Figure 6.10: Numerical results of the turbulence kinetic energy in the OptiChamber ${ }^{\circledast}$ spacer at $30 \mathrm{~L} / \mathrm{min}$

Figure 6.11 and 6.12 demonstrate the development of the velocity profile across the domain for the Volumatic ${ }^{\mathrm{TM}}$ and OptiChamber ${ }^{\circledR}$, respectively, at a flow rate of 30 $\mathrm{L} / \mathrm{min}$ over the first $110 \mathrm{~ms}$ of the run. As illustrated, regions of higher velocity tend to be towards the bottom half of both spacers. This is due to the downwards spray angle and will result in particles with higher inertia impacting the bottom regions of the spacer. For the Volumatic ${ }^{\mathrm{TM}}$ spacer, the jet remains confined to the proximal half of the spacer device. Conversely, for the OptiChamber ${ }^{\circledR}$ spacer, the jet comes close to the midplane of the spacer which could promote distal deposition of the particulate. 


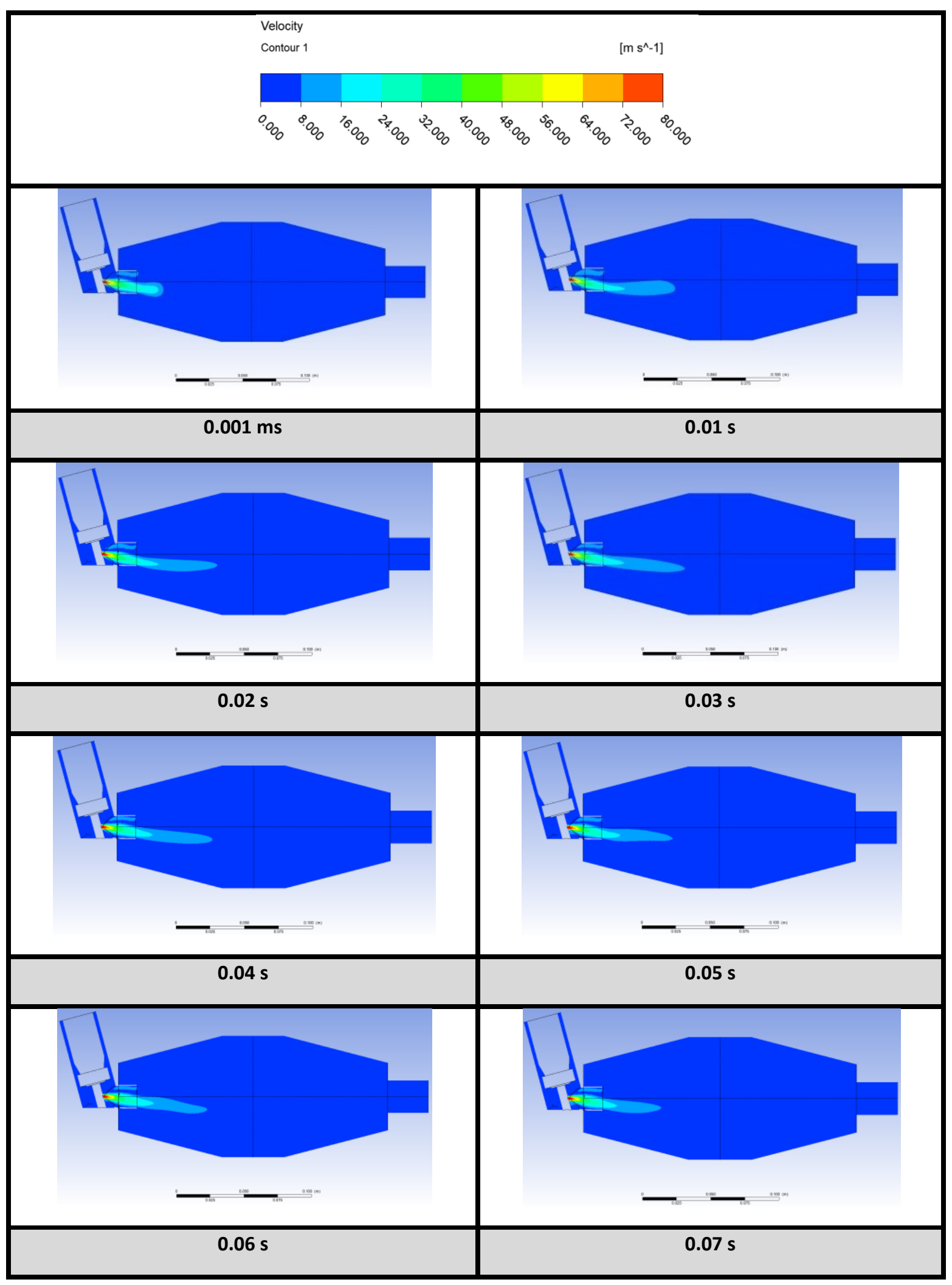




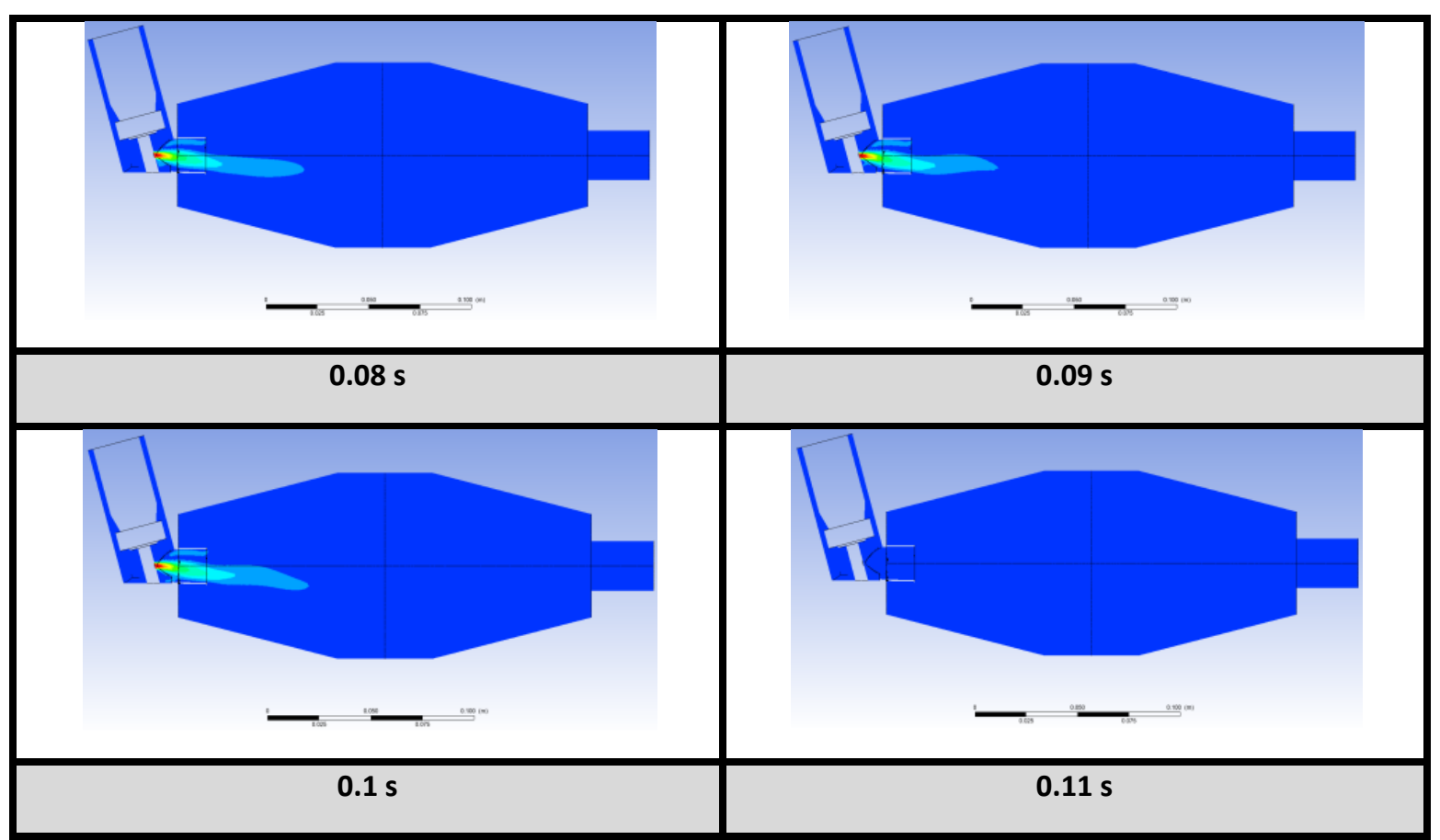

Figure 6.11: Numerical results of the velocity in the Volumatic ${ }^{\mathrm{TM}}$ spacer at $30 \mathrm{~L} / \mathrm{min}$

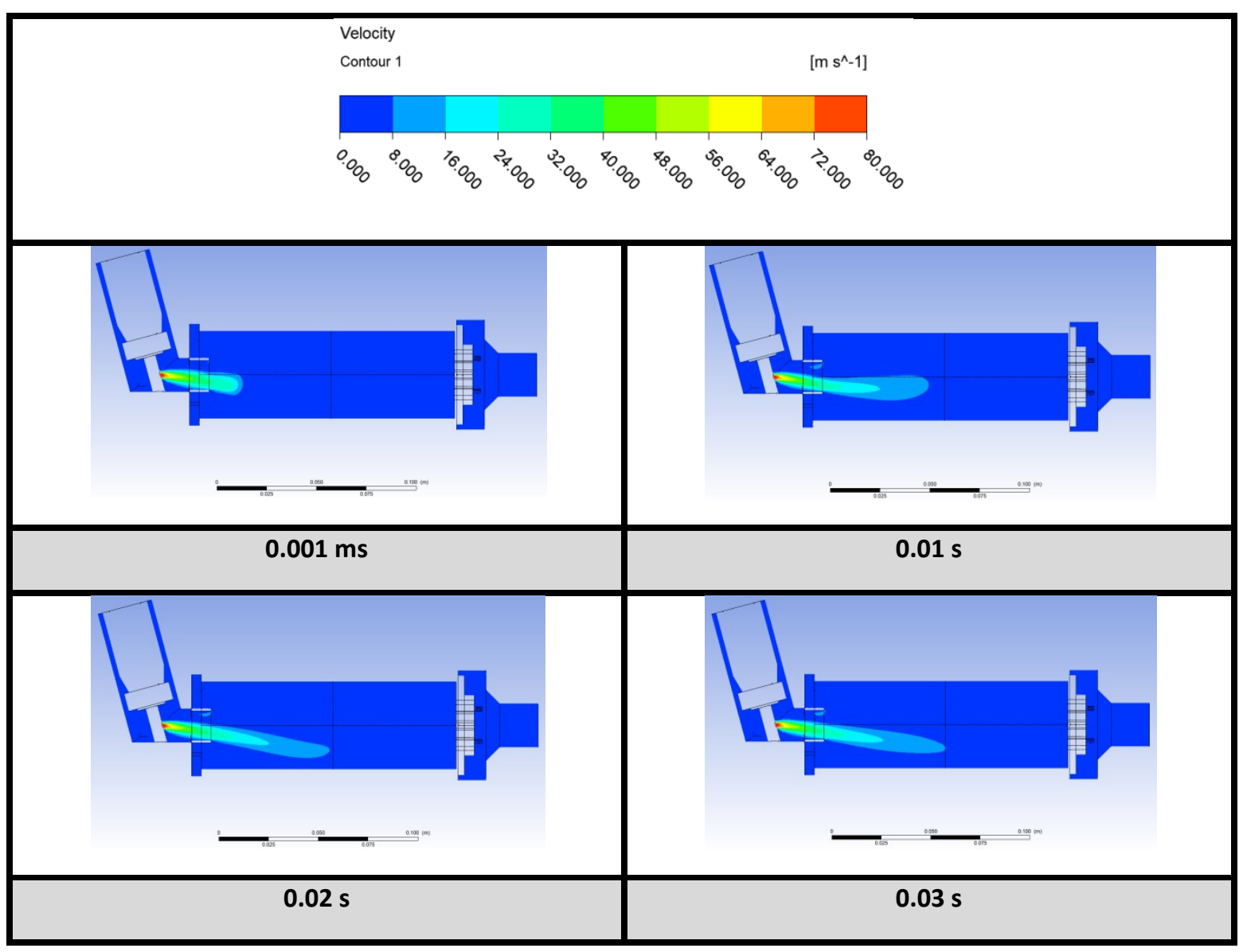




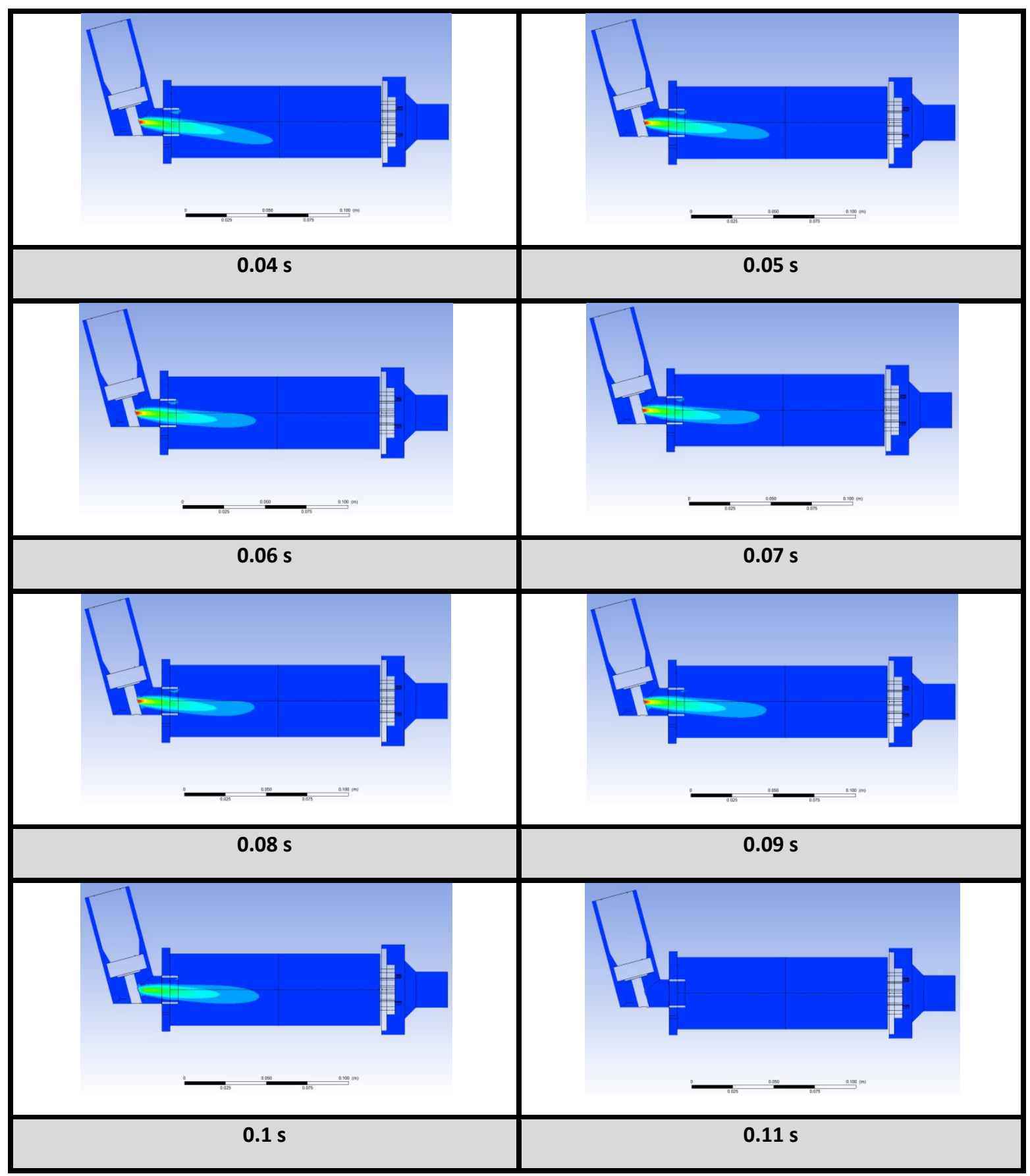

Figure 6.12: Numerical results of the velocity in the OptiChamber ${ }^{\circledR}$ spacer at $30 \mathrm{~L} / \mathrm{min}$

\subsection{Discussion}

Based on the findings of the numerical analysis, the deposition mechanism for the Volumatic ${ }^{\mathrm{TM}}$ and OptiChamber ${ }^{\circledR}$ spacer can be assessed. Considering the results of 
the turbulent tracking simulations (Figure 6.7 and 6.8), both the Volumatic ${ }^{\mathrm{TM}}$ and OptiChamber ${ }^{\circledR}$ spacer demonstrated a penetration similar to the experimental results. Numerical results for the Volumatic ${ }^{\mathrm{TM}}$ spacer demonstrated a slightly lower amount of medication reaching the patient. This implies that while turbulent tracking did better demonstrate the amount of medication reaching the patient than mean flow tracking, the turbulence effects seem to have been exaggerated. Over prediction of particle deposition from turbulent tracking models were found by Matida et al. [72]. As such, a near wall correction may be required for better agreement with experimental results. Conversely, numerical results for the OptiChamber ${ }^{\circledast}$ spacer demonstrated a slightly larger amount of medication reaching the patient when compared to experimental values. This implies that the turbulence effects were lower than expected, and a larger eddy viscosity ratio may be beneficial for the numerical analysis of the OptiChamber ${ }^{\circledR}$ spacer. It is important to note, however, that if the eddy viscosity ratio is increased, it must be increased for all simulations (including those for the Volumatic ${ }^{\mathrm{TM}}$ spacer). As such, this may be considered an ad hoc approach to adjust results. However, by including a near wall correction factor in both simulations, the effects of turbulence on deposition may be adjusted in a much more fundamental manner.

Comparing the results of the mean flow tracking simulations (Figure 6.5 and 6.6) to experimental data (Figure 6.2), the deposition patterns seemed to vary greatly from those found experimentally. Numerical results demonstrated a much larger amount of inhaled medication and a vast under prediction of the amount of medication depositing 
in the spacer itself. As such, for both spacers, it seemed deposition is more likely due to turbulence in the flow as opposed to inertial impaction.

Experimentally, for both spacers, it is interesting to note that the percentage of delivered medication (i.e. that deposited in the filter) was only increased by about $2 \%$ when the flow rate was doubled from $30 \mathrm{~L} / \mathrm{min}$ to $60 \mathrm{~L} / \mathrm{min}$. To double an inhalation flow rate, a patient must inhale more quickly and deeply, which requires quite a significant amount of effort. From Figure 6.3, it is apparent that a larger amount of distal deposition is expected at higher flow rates than proximal deposition. It is possible that a greater flow rate (i.e. larger and deeper breaths by the patient) will only allow for a slight increase in the delivered medication, with the majority of the entrained medication depositing in the distal regions without exiting the spacer. The amount of deposited medication would essentially be shifted from the proximal to distal half of the spacer due to the higher flow rate, which could provide an explanation as to why the amount of delivered medication remained relatively consistent despite variations in the flow rate.

It is interesting to consider that spacers may have an optimal flow rate at which a suitable amount of medication is delivered with minimal effort. These optimal flow rates can vary from spacer to spacer. To illustrate this point, consider with optimal technique a pMDI delivers $8.7 \%$ of its emitted dose to the lungs and a spacer delivers $20.9 \%$ as found by Newman et al. [73]. A patient's daily dose required is $100 \mathrm{mcg}$ of salbutamol sulphate. As such, a patient is prescribed four puffs a day to reach the required dosage with the pMDI alone. 
Using the Volumatic ${ }^{\mathrm{TM}}$ spacer at $30 \mathrm{~L} / \mathrm{min}, 85.52 \%$ (from Figure 6.1) of the medication will be delivered from the spacer and $20.9 \%$ of this dosage will be delivered to the lungs. So, from four doses using the Volumatic ${ }^{\mathrm{TM}}$ spacer at $30 \mathrm{~L} / \mathrm{min}$, the patient is delivered $71.5 \mathrm{mcg}$ of salbutamol sulphate. Now consider if this patient were to inhale with a flow rate of $60 \mathrm{~L} / \mathrm{min}$. At $60 \mathrm{~L} / \mathrm{min}$, the Volumatic ${ }^{\mathrm{TM}}$ spacer delivers $87.01 \%$ of the medication and $20.9 \%$ of this is delivered to the lungs. So, the patient will be delivered $72.7 \mathrm{mcg}$ of salbutamol sulphate. Comparing the amount of medication delivered using an inhalation flow rate of $30 \mathrm{~L} / \mathrm{min}$ versus $60 \mathrm{~L} / \mathrm{min}$, one can see the patient received only a mere $1.2 \mathrm{mcg}$ more by doubling their inhalation effort. As such, the required effort to double an inhalation flow rate is not necessarily required to meet dosage requirements. The same can be illustrated with the OptiChamber ${ }^{\circledR}$ as summarized in Table 6.3.

As demonstrated, the difference in medication delivery between an inhalation flow rate of $30 \mathrm{~L} / \mathrm{min}$ and $60 \mathrm{~L} / \mathrm{min}$ was minimal for both spacers. As such, it is presumed that the extra effort required to achieve a larger inhalation flow rate is redundant as it will have a minimal impact on the delivered dosage. This is especially pertinent for patients with pre-existing respiratory conditions or children who may have difficulties achieving a large inhalation flow rate. It seems that only when the medication delivery of a spacer varies greatly between flow rates (i.e. much more than $2 \%)$, the inhalation flow rate becomes significant and will have a much larger impact on the delivered dosage. As such, it is suggested that spacer devices should be studied in 
more detail to determine the optimal flow rate at which a suitable amount of medication is delivered with minimal effort.

Table 6.3: Hypothetical dose delivery with 0 ptiChamber ${ }^{\circledR}$ at $30 \mathrm{~L} / \mathrm{min}$ versus $60 \mathrm{~L} / \mathrm{min}$

Four doses delivered at $30 \mathrm{~L} / \mathrm{min}$ with OptiChamber
Four doses delivered at $60 \mathrm{~L} / \mathrm{min}$ with OptiChamber
Difference in dose between flow rates with OptiChamber

$41.5 \mathrm{mcg}$

$44.2 \mathrm{mcg}$

$2.6 \mathrm{mcg}$

Based on the experimental and numerical analysis conducted, it is interesting to consider the effect of the valve on the distal end of a spacer. For a patient who is familiar and well practiced with the synchronization of pMDI actuation and inhalation, as well as able to achieve the inhalation flow rate to open a valve, it would be beneficial for a spacer to not have a valve or filter on its distal end but rather, an opening with a large enough diameter to allow the medication to exit the spacer with minimal disturbance (i.e. reduce the sharpness in the degree of convergence to a nozzle) and deposition. At higher flow rates, a valve or filter would only act to impinge medication and reduce the amount delivered. However, for patients who are not as practiced with proper technique, the addition of a valve can help retain medication in the holding chamber to be later inhaled if the pMDI actuation and inhalation were asynchronous. In this situation, a valve is still beneficial although it causes medication losses.

In addition to determining whether a valve is required, spacer design would also benefit from recognizing the downward spray angle of the pMDI. A spacer that 
considered the $-6^{\circ}$ deflection in its design would results in less deposition in the lower portion of the spacer. This could be achieved by implementing a nonsymmetrical design of the spacer itself, curving the bottom portion to provide an increased distance between the nozzle of the pMDI and the bottom surface of the spacer. A bulbous spacer which does not interrupt the streamlines of the medication would be more beneficial than one with sharp edges.

\subsection{Conclusions}

In this study, measurements of the regional deposition of a pMDI aerosol spray, salbutamol sulphate, were conducted on a medium- and large- volume spacer, namely, the OptiChamber ${ }^{\circledR}$ and Volumatic ${ }^{\top \mathrm{M}}$ spacer. For both spacers, it was found that an increase in the inhalation flow rate from 30 to $60 \mathrm{~L} / \mathrm{min}$ only resulted in a slight increase (about 2-3\%) in the amount of medication provided to the patient. Experimental results demonstrated that at higher inhalation flow rates, more deposition will be expected in the distal portion of the spacers as opposed to the proximal portion. Furthermore, a larger amount of medication was found to deposit on the bottom surface than the top surface of both spacers due to the $-6^{\circ}$ deflection of the pMDI spray. These differences were confirmed to be statistically different by a correlated t-test. It seemed that a variation of flow rate will act to shift the location and amount of deposition in the spacer, and not necessarily provide more medication to the patient.

In addition to experimental work, a numerical study was carried out by applying both mean flow and turbulent particle tracking using URANS equations with a SST 
turbulence model. Simulations were conducted at an inhalation flow rate of $30 \mathrm{~L} / \mathrm{min}$ implementing an experimental monodisperse particle size distribution for salbutamol sulphate of Alatrash [70]. This numerical analysis confirmed that more medication can be expected to deposit on the bottom surface of the spacers due to the downward spray deflection of the pMDI. Numerical results also demonstrated the mechanism of deposition of both the Volumatic ${ }^{\mathrm{TM}}$ and OptiChamber ${ }^{\circledR}$ spacer. The numerical analysis of the spacers using mean flow tracking, demonstrated an over prediction of penetration with minimal deposition in the spacer itself. Conversely, with the implementation of turbulent tracking, penetration and deposition patterns seemed to better reflect experimental results for both spacers. This suggested that turbulence is likely to be the dominant method of deposition in both the Volumatic ${ }^{\mathrm{TM}}$ and OptiChamber ${ }^{\circledR}$ spacer. $^{\circ}$

\subsection{Future Work}

To develop this research further, numerically, eddies and the behaviour of the flow could be resolved further. To improve numerical results, it seems necessary to resolve and capture the effect which eddies have on the micron sized particulate in the fluid flow. During the course of this study, a DES analysis was attempted with 10 million element mesh, however, results proved inconclusive. It is conjectured that a finer mesh (upwards of 20 million elements) is required to resolve the eddies and their influence on the particulate fully. A full LES analysis could be completed to demonstrate the impact of eddies and turbulence on particulate deposition as well as observe the interaction of particles with those eddies as they travel through the spacer devices. This work could be 
extended to include additional inhalation flow rates as well. Furthermore, a near wall correction factor could be investigated to reduce the over-prediction of wall deposition of micron sized particulate when implementing turbulent tracking methods as well as an adjustment of the eddy viscosity ratio for both spacers.

Results from the numerical analysis using CFD suggest that turbulence effects are of great importance to the deposition found inside the tested spacer devices. Ideally, if regions of high turbulence could be minimized through design changes to the spacers, less deposition due to turbulence effects would be expected. These design changes could be demonstrated through a generational approach by simulating different spacer designs which would promote and exclude certain design features to reduce the highly turbulent areas of the spacer devices (particularly close to the nozzle of the pMDI). Difficulties arise, however, in running the amount of simulations required for this generational analysis. Due to the transient nature of the pMDI actuation and development of the spray angle inside the spacer device, simulations are lengthy and would require several simplifications to optimize the process.

Experimentally, medication deposition in additional spacers could be analyzed with the same regional approach (i.e. the division of the spacers into four quadrants). Furthermore, a dynamic flow rate could be implemented to closer simulate the proper inhalation technique recommended for a patient while using a spacer (i.e. the patient should inhale with a slow, deep breath followed by a breath-hold). This study also involved the use of a steady inhalation flow rate through the experimental apparatus. Ideally, a patient would synchronize inhalation and the actuation of the pMDI. If there is 
delay during the actuation of the pMDI and inhalation, particulate could begin to settle, and deposition values would increase. As such, it would be interesting to observe the effects of deposition on exact and delayed synchronization. Furthermore, spacers with a valve typically require a specific flow rate to open the valve. In this study, it was assumed that flow rates of $30 \mathrm{~L} / \mathrm{min}$ and above were sufficient to completely open the valve. Future experimental work could include a study of the valve behaviour under various flow rates as well as delayed inhalation. If the valve was not completely open, more deposition would be expected in the distal regions of the spacer. 


\section{Chapter 7: References}

[1] Statistics Canada, "Chronic Conditions, 2016," 27 September 2017. [Online]. Available: https://www150.statcan.gc.ca/n1/en/pub/82-625-x/2017001/article/54858-eng.pdf?st=Ge10Hqi. [Accessed 10 September 2019].

[2] Public Health Agency of Canada, "Report from the Canadian Chronic Disease Surveillance System: Asthma and Chronic Obstructive Pulmonary Disease (COPD) in Canada, 2018," March 2018. [Online]. Available: http://publications.gc.ca/collections/collection_2018/aspc-phac/HP35-90-2018-eng.pdf. [Accessed 10 September 2019].

[3] L. Akinbami, C. Bailey, C. Johnson, M. King, X. Liu, J. Moorman and H. Zahran, "Trends in asthma prevalence, health care use, and mortality in the United States, 2001-2010," 2012.

[4] L. J. Akinbami, J. E. Moorman and X. Liu, "Asthma prevalence, health care use, and mortality; United States, 2005-2009," 2011.

[5] S. P. Newman, "Spacer devices for metered dose inhalers," Clinical pharmacokinetics, vol. 43, no. 6, pp. 349-360, 2004.

[6] NPS MedicineWise, "Asthma treatment: how to use a metered-dose inhaler (multiple breath) with a spacer," YouTube, 23 August 2012. [Online]. Available: https://www.youtube.com/watch?v=GSUOiD9xwwo. [Accessed 23 September 2020].

[7] P. W. Barry and C. O'Callaghan, "Inhalational drug delivery from seven different spacer devices," Thorax, vol. 51, no. 8, pp. 835-840, 1996.

[8] S. Newman and M. Newhouse, "Effect of add-on devices for aerosol drug delivery: deposition studies and clinical aspects," Journal of aerosol medicine, vol. 9, no. 1, pp. 55-70, 1996.

[9] M. Vidgren, T. Paronen, A. Kärkkäinen and P. Karjalainen, "Effect of extension devices on the drug deposition from inhalation aerosols," International journal of pharmaceutics, vol. 39, no. 1-2, pp. 107-112, 1987.

[10] H. Matthys, "Inhalation delivery of asthma drugs," Lung, vol. 168, no. 1, pp. 645-652, 1990.

[11] C. O'Callaghan, J. Lynch, M. Cant and C. Robertson, "Improvement in sodium cromoglycate delivery from a spacer device by use of an antistatic lining, immediate inhalation, and avoiding multiple actuations of drug," Thorax, vol. 48, no. 6, pp. 603-606, 1993.

[12] H. Chrystyn, "Methods to identify drug deposition in the lungs following inhalation," British 
journal of clinical pharmacology, vol. 51, no. 4, p. 289, 2001.

[13] N. Snell and D. Ganderton, "Assessing lung deposition of inhaled medications: Consensus statement from a workshop of the British Association for Lung Research," Respiratory medicine, vol. 93, no. 2, pp. 123-133, 1999.

[14] R. Pirie, B. McGorum, C. Owen, O. Carr, H. Oakley and G. McLachlan, "Factors affecting the efficiency of aerosolized salbutamol delivery via a metered dose inhaler and equine spacer device," Journal of veterinary pharmacology and therapeutics, vol. 40, no. 3, pp. 231-238, 2017.

[15] G. Alhegagi, "Pressurized Metered-dose Inhaler (pMDI) Aerosol Deposition Measurements in Add-on Spacers," Carleton University, Ottawa, 2009.

[16] E. Sprigge, "Non uniform deposition of pMDI aerosol in a large volume spacer," Carleton University, Ottawa, 2014.

[17] J. P. Ward, J. Ward and R. M. Leach, The respiratory system at a glance, John Wiley \& Sons, 2015.

[18] B. Maury, The Respiratory System in Equations, Springer Milan, 2013.

[19] M. C. lonescu, The Human Respiratory System: An Analysis of the Interplay between Anatomy, Structure, Breathing and Fractal Dynamics, Springer London, 2013.

[20] Statistics Canada, "Asthma, by age group (Table 13-10-0096-08)," Government of Canada, 2019.

[21] Statistics Canada, "Chronic obstructive pulmonary disease (COPD), 35 years and over (Table 13-10-0096-19)," Government of Canada, 2019.

[22] Cystic Fibrosis Canada, "The Canadian Cystic Fibrosis Registry 2017 Annual Data Report," 2018.

[23] A. Papi, C. Brightling, S. Pedersen and H. Reddel, "Asthma," The Lancet, vol. 391, no. 10122, pp. 783-800, 2018.

[24] Statistics Canada, "Life and Breath: Respiratory Disease in Canada," Government of Canada, 2007.

[25] R. Gunderman and B. Lydon, "Respiratory disease: an update for radiologists," Academic radiology, vol. 23, no. 1, pp. 108-111, 2016.

[26] Healthcare-Online, "Home Remedies for Asthma," 3 October 2019. [Online]. Available: http://www.healthcare-online.org/Home-Remedies-For-Asthma.html. [Accessed 3 October 
2019].

[27] J. Quirt, K. Hildebrand, J. Mazza, F. Noya and H. Kim, "Asthma," Allergy, Asthma \& Clinical Immunology, vol. 14, no. 2, pp. 1-16, 2018.

[28] J. Tu, K. Inthavong and G. Ahmadi, Computational fluid and particle dynamics in the human respiratory system, Springer Science \& Business Media, 2012, pp. 19-44.

[29] A. Aliverti and P. Antonio, Mechanics of Breathing: New Insights from New Technologies, Springer Milan, 2014.

[30] R. Pauwels, A. Buist, P. Calverley, C. Jenkins and S. Hurd, "Global strategy for the diagnosis, management, and prevention of chronic obstructive pulmonary disease: NHLBI/WHO Global Initiative for Chronic Obstructive Lung Disease (GOLD) Workshop summary," American journal of respiratory and critical care medicine, vol. 163, no. 5, pp. 1256-1276, 2001.

[31] K. F. Rabe and H. Watz, "Chronic obstructive pulmonary disease," The Lancet, vol. 389, pp. 1931-1940, 2017.

[32] D. R. Hess, "Aerosol delivery devices in the treatment of asthma," Respiratory care, vol. 53, no. 6 , pp. 699-725, 2008.

[33] B. K. Rubin and J. B. Fink, "Optimizing aerosol delivery by pressurized metered-dose inhalers," Respiratory Care, vol. 50, no. 9, pp. 1191-1200, 2005.

[34] Copley Scientific, "Measuring the cold freon effect," Inhalation, 2012.

[35] K. J., S. D. R., B. J. R., K. M. D. and M. A. R., "Aerosolization of bacterial spores with pressurized metered dose inhalers," Aerosol Science and Technology, vol. 47, no. 10, pp. 1108-1117, 2013.

[36] P. Myrdal, P. Sheth and S. Stein, "Advances in metered dose inhaler technology: formulation development," AAPS PharmSciTech, vol. 15, no. 2, pp. 434-455, 2014.

[37] R. Dalby, M. Spallek and T. Voshaar, " A review of the development of Respimat ${ }^{\circledR}$ Soft Mist $^{\mathrm{TM}}$ Inhaler," International journal of pharmaceutics, vol. 283, no. 1-2, pp. 1-9, 2004.

[38] Boehringer Ingelheim, "Canadians with Chronic Obstructive Pulmonary disease lack confidence with inhaler: 6 in 10 report challenges," Boehringer Ingelheim, 30 March 2015. [Online]. Available: https://www.boehringer-ingelheim.ca/en/press-release/canadianschronic-obstructive-pulmonary-disease-lack-confidence-inhaler-6-10-report. [Accessed 4 November 2019].

[39] H. Wachtel, S. Kattenbeck, S. Dunne and B. Disse, "The Respimat ${ }^{\circledR}$ development story: 
patient-centered innovation," Pulmonary Therapy, vol. 3, no. 1, pp. 19-30, 2017.

[40] B. Zierenberg and J. Eicher, "The Respimat, a new soft mist inhaler for delivering drugs to the lungs," Modified-release drug delivery technology, pp. 949-958, 2002.

[41] Government of Canada, "Voluntary Compliance Undertaking of Boehringer Ingelheim (Canada) Ltd. to the Patented Medicine Prices Review Board," Patented Medicine Prices Review Board, 26 May 2016. [Online]. Available: https://www.pmprbcepmb.gc.ca/view.asp?ccid=1266\&lang=en. [Accessed 4 November 2019].

[42] Government of Canada, "Report on New Patented Drugs - Spiriva," Patented Medicine Prices Review Board, 6 February 2017. [Online]. Available: http://www.pmprbcepmb.gc.ca/view.asp?ccid=655. [Accessed 4 November 2019].

[43] J. Budzinski, Interviewee, MDI Cost. [Interview]. 15 July 2020.

[44] D. Prime, P. J. Atkins, A. Slater and B. Sumby, "Review of dry powder inhalers," Advanced drug delivery reviews, vol. 26, no. 1, pp. 51-58, 1997.

[45] R. N. P. Ltd, "The Global Dry Powder Inhaler Market is Anticipated to Post a Noteworthy CAGR By the End of 2024," 23 January 2018. [Online]. Available: https://www.openpr.com/news/913501/the-global-dry-powder-inhaler-market-isanticipated-to-post-a-noteworthy-cagr-by-the-end-of-2024.html. [Accessed 23 September 2020].

[46] M. J. Telko and A. J. Hickey, "Dry powder inhaler formulation," Respiratory care, vol. 50, no. 9, pp. 1209-1227, 2005.

[47] A. M. Fonceca, W. G. F. Ditcham, M. L. Everard and S. Devadason, "Drug Administration by Inhalation in Children," Kendig's Disorders of the Respiratory Tract in Children, pp. 257-271, 2019.

[48] C. O'Callaghan and P. W. Barry, "The science of nebulised drug delivery," Thorax, vol. 52, no. Suppl 2, 1997.

[49] W. Vincken, M. L. Levy, J. Scullion, O. S. Usmani, P. R. Dekhuijzen and C. J. Corrigan, "Spacer devices for inhaled therapy: why use them, and how?," ERJ open research, vol. 4, no. 2, 2018.

[50] C. O'Callaghan, J. Lynch, M. Cant and C. Robertson, "Improvement in sodium cromoglycate delivery from a spacer device by use of an antistatic lining, immediate inhalation, and avoiding multiple actuations of drug," Thorax, vol. 48, no. 6, pp. 603-606, 1993.

[51] P. Demoly, P. Hagedoorn, A. H. de Boer and H. W. Frijlink, "The clinical relevance of dry powder inhaler performance for drug delivery," Respiratory medicine, vol. 108, no. 8, pp. 
1195-1203, 2014.

[52] J. Borghardt, C. Kloft and A. Sharma, "Inhaled Therapy in Respiratory Disease: The Complex Interplay of Pulmonary Kinetic Processes," Canadian Respiratory Journal, vol. 2018, 2018.

[53] O. S. Usmani, M. F. Biddiscombe and P. J. Barnes, "Regional lung deposition and bronchodilator response as a function of $\beta 2$-agonist particle size," American journal of respiratory and critical care medicine, vol. 172, no. 12, pp. 1497-1504, 2005.

[54] C. Darquenne, "Aerosol deposition in health and disease," Journal of aerosol medicine and pulmonary drug delivery, vol. 25, no. 3, pp. 140-147, 2012.

[55] K. W. Nicholson, "A review of particle resuspension," Atmospheric Environment (1967), vol. 22, no. 12, pp. 2639-2651, 1988.

[56] G. Alfonsi, "Reynolds-averaged navier-stokes equations for turbulence modeling," Applied Mechanics Reviews, vol. 62, no. 4, 2009.

[57] R. W. Johnson, "Modeling strategies for unsteady turbulent flows in the lower plenum of the VHTR," Nuclear Engineering and Design, vol. 238, no. 3, pp. 482-491, 2008.

[58] J. E. Bardina, P. G. Huang and T. J. Coakley, "Turbulence modeling validation, testing, and development," 1997.

[59] T. Thatcher and W. Nazaroff, "Effect of small-scale obstructions and surface textures on particle deposition from natural convection flow," Aerosol Science and Technology, vol. 27, no. 6, pp. 709-725, 1997.

[60] Brooks Instrument, "Brooks ${ }^{\circledR}$ Mass Flow Meter Model 5863 Mass Flow COntroller Model 5853 Installation and Operation Manual," Brooks Instrument, 2008.

[61] Agilent Technologies, "Agilent 8453 UV-visible spectrophotometer - UV-visible spectroscopy solutions for chemical analysis," Agilent Technologies, 2008.

[62] C. De Caro, "UV/VIS spectrophotometry-fundamentals and applications," Mettler-Toledo International, 2015.

[63] G. Alhegagi and E. Matida, "Non-symmetrical pMDI deposition measurement in add-on spacers [Abstract]," in American Association for Aerosol Research 29th Annual Conference, Portland, Oregon, 2010.

[64] A. Mishra, M. Kumar, A. Mishra, A. Verma and P. Chattopadhyay, "Validated UV spectroscopic method for estimation of salbutamol from tablet formulations," Archives of Applied Science Research, vol. 2, no. 3, pp. 207-211, 2010. 
[65] C. Kumaresan and K. Sathishkumar, "Development of an inhaled sustained release dry powder formulation of salbutamol sulphate, an antiasthmatic drug," Indian journal of pharmaceutical sciences, vol. 78, no. 1, p. 136, 2016.

[66] R. Cook and R. Jankow, "Effects of stray light in spectroscopy," Journal of Chemical Education, vol. 49, no. 6, p. 405, 1972.

[67] A.S.M.E., "PTC 19.1-2005 Test Uncertainty," American Society of Mechanical Engineers, vol. 3, pp. 10016-5990, 2006.

[68] Agilent Technologies, "Specifications-8453 UV-Visible spectrophotometer," 1993.

[69] B. Crosland, M. Johnson and E. Matida, "Characterization of the spray velocities from a pressurized metered-dose inhaler," Journal of aerosol medicine and pulmonary drug delivery, vol. 22, no. 2, pp. 85-98, 2009.

[70] A. Alatrash, "Experimental and numerical study of inhaler spray characterization (size distributions and velocity)," Carleton University, Ottawa, 2018.

[71] O. Gryczka, S. Heinrich, N. Deen, M. van Sint Annaland, J. Kuipers, M. Jacob and L. Mörl, "Characterization and CFD-modeling of the hydrodynamics of a prismatic spouted bed apparatus," Chemical engineering science, vol. 64, no. 14, pp. 3352-3375, 2009.

[72] E. A. Matida, W. H. Finlay, C. F. Lange and B. Grgic, "Improved numerical simulation of aerosol deposition in an idealized mouth-throat," Journal of Aerosol Science, vol. 35, no. 1, pp. 1-19, 2004.

[73] S. P. Newman, A. B. Millar, T. R. Lennard-Jones, F. O. L. K. E. Morén and S. W. Clarke, "Improvement of pressurised aerosol deposition with Nebuhaler spacer device," Thorax, vol. 39, no. 12, pp. 935-941, 1984.

[74] D. A. Mahler, "Peak Inspiratory Flow Rate: An Emerging Biomarker in Chronic Obstructive Pulmonary Disease," American journal of respiratory and critical care medicine, vol. 199, no. 12, pp. 1577-1579, 2019.

[75] P. Haidl, S. Heindl, K. Siemon, M. Bernacka and R. Cloes, "Inhalation device requirements for patients' inhalation maneuvers," Respiratory medicine, vol. 118, pp. 65-75, 2016.

[76] B. Johal, M. Howald, M. Fischer, J. Marshall and G. Venthoye, "Fine particle profile of fluticasone propionate/formoterol fumarate versus other combination products: the DIFFUSE study," Combination Products in Therapy, vol. 3, no. 1-2, pp. 39-51, 2013.

[77] J. Mitchell, M. Nagel, K. Wiersema, S. Bates, R. Morton and J. Schmidt, "Performance of large and small volume valved holding chambers (VHCs) as a function of flow rate," Drug Deliv. Lungs-XI., pp. 52-55, 2000. 
[78] B. Laube, H. Janssens, F. de Jongh, S. Devadason, R. Dhand, P. Diot, M. Everard, I. Horvath, P. Navalesi, T. Voshaar and H. Chrystyn, "What the pulmonary specialist should know about the new inhalation therapies.," European Respiratory Journal, vol. 37, no. 6, p. 1308-1331, 2011.

[79] M. Corradi, H. Chrystyn, B. Cosio, M. Pirozynski, S. Loukides, R. Louis, M. Spinola and O. Usmani, "NEXThaler, an innovative dry powder inhaler delivering an extrafine fixed combination of beclometasone and formoterol to treat large and small airways in asthma. Expert opinion on drug delivery," Expert opinion on drug delivery, vol. 11, no. 9, pp. 14971506, 2014.

[80] B. Lipworth, "Pharmacokinetics of inhaled drugs," British journal of clinical pharmacology, vol. 42, no. 6, pp. 697-705, 1996.

[81] B. J. Lipworth, "Modern drug treatment of chronic asthma," BMJ, vol. 318, no. 7180, pp. 380-384, 1999.

[82] H. S. Nelson, " $\beta$-Adrenergic bronchodilators," New England Journal of Medicine, vol. 333, no. 8, pp. 499-507, 1995.

[83] Y. Zhiyin, "Large-eddy simulation: Past, present and the future," Chinese journal of Aeronautics, vol. 28, no. 1, pp. 11-24, 2015. 


\section{Chapter 8: Appendices}

\section{Appendix A: Uncertainty Data}

Table 8.1: Uncertainty values

\begin{tabular}{cccc}
\hline \multicolumn{2}{c}{ Volumatic Spacer } & \multicolumn{2}{c}{ OptiChamber Spacer } \\
\cline { 1 - 2 } Uncertainty & $\underline{\text { Value }}$ & $\underline{\text { Uncertainty }}$ & Value \\
$B_{a, 1}$ & $43.864 \mathrm{mcg}$ & $B_{a, 1}$ & $43.864 \mathrm{mcg}$ \\
$S_{a, 1}$ & $13.892 \mathrm{mcg}$ & $S_{a, 1}$ & $107.964 \mathrm{mcg}$ \\
$B_{b, 1}$ & $0 \mathrm{mcg}$ & $B_{b, 1}$ & $0 \mathrm{mcg}$ \\
$S_{b, 1}$ & $6.946 \mathrm{mcg}$ & $S_{b, 1}$ & $53.982 \mathrm{mcg}$ \\
$B_{c, 1}$ & $21.932 \mathrm{mcg}$ & $B_{c, 1}$ & $21.932 \mathrm{mcg}$ \\
$S_{c, 1}$ & $6.946 \mathrm{mcg}$ & $S_{c, 1}$ & $53.982 \mathrm{mcg}$ \\
$B_{d, 1}$ & $49.041 \mathrm{mcg}$ & $B_{d, 1}$ & $49.041 \mathrm{mcg}$ \\
$S_{d, 1}$ & $17.014 \mathrm{mcg}$ & $S_{d, 1}$ & $132.228 \mathrm{mcg}$ \\
$\frac{S_{T P}}{T P}$ & 0.0275 & $\frac{S_{T P}}{T P}$ & 0.0558 \\
$\frac{B_{T P}}{T P}$ & 0.0791 & $\frac{B_{T P}}{T P}$ & 0.0207 \\
& & & \\
\hline
\end{tabular}




\section{Appendix B: Supplementary Background Information}

\section{B.1 Respiratory System Physiology}

Each organ and structure of the respiratory system serves a different physiological purpose and, as such, has an appropriate anatomy to fulfill its purpose. Beginning with the nose and naval cavity, these organs and structures serve as a passageway for air to pass through to the lungs. Additionally, they also warm, humidify, and clean (of foreign particles) the air as well as provide sounds for speech and house the olfactory receptors used for smell. The pharynx serves as a passageway to the digestive and respiratory system, allowing both air and food to pass through it. The epiglottis prevents swallowed food from entering the larynx by acting as a lid which covers the trachea. Additionally, the larynx further contributes to the removal of foreign particles and the warming and humidification of inhaled air. The larynx also serves to aid in sound generation by manipulating pitch and volume via the flexing and reflexing of the muscles in the larynx to control the movement of the vocal cords. Finally, the bronchial tree and lungs act to conduct inhaled air to and from the alveoli [28].

\section{B.1.1 Lung Capacity}

It is important to understand lung function and capacity when considering how medication is delivered to the lungs via inspiration. Lung volumes and inhalation rates could influence the delivered dosage through a spacer device. Typically, lung volumes are determined using a spirometer, a body plethysmograph, or an X-ray. Spirometers measure the inspiration and expiration of air from the lungs. Spirometer can be either 
dynamic or static. Dynamic spirometry involves the measurement of flow or volume over time, whereas static spirometry involves the measurement of only gas volume. The lung capacity can be inferred from these measurements as well as the presence of obstructions or restrictions. An obstruction reduces the ability to exhale all the air in the lungs and is typically present in pulmonary disorders such as asthma and chronic obstructive pulmonary disease (COPD). A restriction, on the other hand, causes difficulty with fully filling the lungs on an inhalation. This is indicated by inspiration difficulty, rapid, and shallow breathing [29].

A number of parameters and capacities are important to defining lung function as listed in Table 8.2 .

Table 8.2: Lung parameters (Adapted from [28])

\begin{tabular}{|c|c|c|}
\hline Term & Acronym & Definition \\
\hline Tidal Volume & TV & $\begin{array}{l}\text { Normal volume of air inhaled and exhaled during breathing } \\
\text { without extra effort. Typically } 8 \text { to } 10 \% \text { of the total lung capacity. }\end{array}$ \\
\hline Inspiratory Reserve Volume & IRV & Volume of air which can be inhaled above normal volume. \\
\hline Expiratory Reserve Volume & ERV & Volume of air which can be exhaled after expiration of normal volume. \\
\hline Residual Volume & $\mathrm{RV}$ & Volume of air remaining after a maximum expiration. \\
\hline Functional Residual Capacity & FRC & Volume of air which can be additionally exhaled after a normal expiration. \\
\hline Inspiratory Capacity & $\mathrm{IC}$ & Maximum volume of air which can be inhaled. \\
\hline Vital Capacity & $\mathrm{VC}$ & Maximum volume of air which can be exhaled and inhaled. \\
\hline Forced Vital Capacity & FVC & $\begin{array}{c}\text { Maximum volume of air which can be forcefully exhaled after a maximum } \\
\text { inhalation. }\end{array}$ \\
\hline Total Lung Capacity & TLC & Maximum volume of the lungs. \\
\hline
\end{tabular}

These parameters can be measured by a spirometer and are demonstrated in Figure 2.5. 


\section{B.1.2 Inspiratory Flow Rate}

In addition to lung capacity parameters, there are a number of other important patient factors which can affect optimal drug delivery including: inspiratory flow rate, flow acceleration rate, time of inhalation, inhaled volume, and breath-hold time [74]. When using a pMDI it is recommended that a patient inhale slowly, however, there is minimal literature which suggests an optimal inspiratory flow rate or a lower limit of an acceptable flow rate [75]. One such factor which affects dosage delivery, the fine particle fraction (FPF), has been found to differ between pMDIs depending on the flow rate. Johal et al. found differences in FPF delivered from various pMDIs at 30 and 60 L/min [76]. This causes an uncertainty as to how high the minimum inhalation flow rate should be.

These same uncertainties apply to pMDIs used with spacer devices as well and little is reported on the minimum required inspiratory flow rate of a pMDI with a spacer [75]. Mitchell et al. compared two valved holding chambers (AeroChamber Plus and Volumatic $\left.{ }^{T M}\right)$ at various flow rates $(28.3,45$, and 60 LPM) with a HFA pMDI formulation. The FPFs were measured using an Andersen 8-stage impactor and were found to be close to $90 \%$ for both spacers. At low flow rates, the FPF of both spacers were comparable while at higher flow rates, they began to differ [77]. Accordingly, this suggested that differences in dosage delivery could be due to flow rate and the optimal flow rate could depend on the pMDI and spacer used.

Overall, literature suggested a varied array of minimum required inspiratory flow rates depending on the type of $\mathrm{pMDI}$, dry powder inhaler (DPI) or spacer used. These 
include: the Autohaler ${ }^{\circledR}$ with a minimum flow rate of $30 \mathrm{~L} / \mathrm{min}$ [78], the Easi-Breathe ${ }^{\circledR}$ with a flow rate of $20 \mathrm{~L} / \mathrm{min}$ [78], the Diskus ${ }^{\circledR}$ with a flow rate of $30 \mathrm{~L} / \mathrm{min}$ [78], the Aerolizer ${ }^{\circledast}$ with a flow rate of greater than $60 \mathrm{~L} / \mathrm{min}$ [78], and the NEXThaler ${ }^{\circledR}$ with a flow rate of $55 \mathrm{~L} / \mathrm{min}[79]$. 


\section{B.2 Inhalation Therapy}

In order to treat respiratory disorders such as asthma and COPD, it is typically recommended that an inhalation therapy is used. Primarily, the goal of inhalation therapy is to reduce pulmonary symptoms (through prevention of airway inflammation and constriction) using a targeted, local treatment method. Typical inhaled drugs include corticosteroids, beta2-adrenoceptor agonists, and antibiotics [80]. These inhaled drugs target the lungs, and due to their targeted approach, lower inhaled doses can be administered and are seen to be therapeutically equivalent (or superior) to higher doses of systemically administered therapies. Additionally, inhalation therapy can allow the drug to have a faster onset than other routes of administration as well as less side effects than those administered orally or intravenously.

Delivery of inhaled drugs is a complex procedure as the lungs are intricate organs with a multifaceted architecture as detailed in Section 2.2. As such, there are numerous pharmacokinetic processes which exist in the pulmonary environment and inhalation route. These processes are additionally influenced by aspects of the inhaled medication such as the drug formulation and use of inhalation device.

Following drug inhalation, the pulmonary pharmacokinetic processes involve the following [52]:

I. Drug particle or droplet deposition

II. Drug dissolution in lung fluids 
III. Drug clearance in conducting airways (via mucociliary clearance) and in the alveolar space (via macrophage clearance)

IV. Absorption of the drug into lung tissue

V. Pulmonary tissue retention of the absorbed/dissolved drug

VI. Drug transport from the lung tissue to blood circulation (via perfusion)

As mentioned, some of the more common inhaled drugs include beta2adrenoceptor agonists. One such drug is salbutamol which is used to treat asthma and acts as a bronchodilator. Salbutamol is a reactive treatment, providing quick relief following an asthma attack.

Beta2-agonists perform by acting on smooth muscle and mast cells to aid in the protection against stimuli of bronchoconstriction [81]. These beta2-agonists can be characterized as either long-acting or short-acting. Short-acting beta2-agonists are typically used occasionally as relief medication while long-acting beta2-agonists are used in ongoing therapy. Beta2-agonists are preferred to relieve acute asthmatic symptoms and in the prevention of exercise-induced bronchospasm [82].

Other inhaled drugs used to treat asthma include corticosteroids which act as anti-inflammatory agents in a non-specific manner. These agents inhibit inflammatory cells, cytokine expression, and transcription factors involved in inflammation [81]. Corticosteroids are typically used in the long-term as a pre-emptive measure to prevent asthma attacks. Examples of corticosteroids include: Fluticasone (Flovent HFA), 
Budesonide (Pulmicort Flexhaler), Mometasone (Asmanex Twisthaler), Beclomethasone (Qvar RediHaler), and Ciclesonide (Alvesco). 


\section{B.3 Large Eddy Simulation}

Large eddy simulation (LES) is an alternative approach to RANS and is used to describe turbulent flows where resolving the large- and small-scale motions of the flow are of importance. LES does not adopt the time- or ensemble-averaging of RANS and the modelling of the Reynolds stress. Instead, LES directly computes the large-scale motions of turbulent flow (i.e. large eddies) and uses models to describe the small-scale flow (i.e. sub-grid scale (SGS)). LES can be seen as more accurate than RANS as it directly computes (as opposed to modelling in RANS) the large eddies involved in turbulent flow which are responsible for most of the momentum transfer and turbulent mixing [83]. LES applies a low-pass spatial filter to the Navier-Stokes equations as only the largescale motions are computed directly. This explicit filtering is illustrated in Figure 8.1, which demonstrates the difference between a filtered velocity $\left(\tilde{u}_{i}\right)$ and instantaneous velocity $\left(u_{x}\right)$.

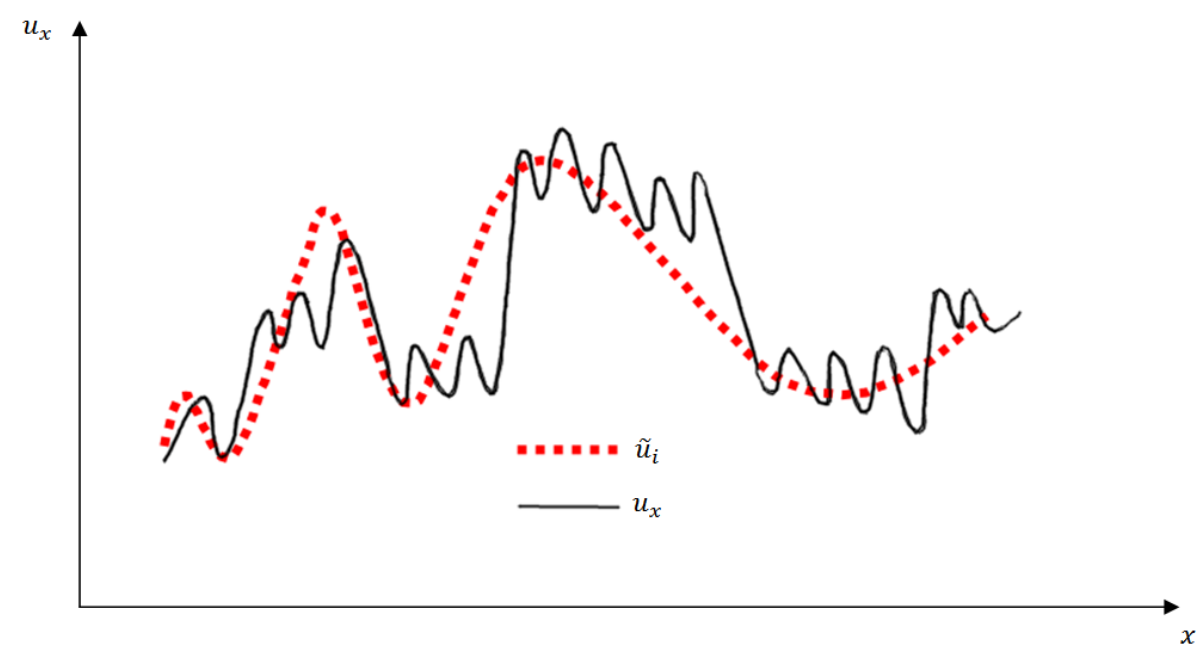

Figure 8.1: Illustration of filtered velocity and instantaneous velocity (Adapted from [83]) 
The filtered Navier-Stokes equations can be expressed as follows,

$$
\frac{\partial \tilde{u}_{i}}{\partial t}+\frac{\partial \tilde{u}_{i}}{\partial x_{j}}\left(\tilde{u}_{j}\right)=-\frac{1}{\rho} \frac{\partial \tilde{p}}{\partial x_{i}}+v \frac{\partial^{2} \tilde{u}_{i}}{\partial x_{j} \partial x_{j}}+\frac{1}{\rho} \frac{\partial \tau_{i j}}{\partial x_{j}}
$$

Where $\tau_{\mathrm{ij}}$ is known as the subgrid stress tensor and contains information of the subgrid fluid velocities. It can be defined as,

$$
\tau_{i j}=\widetilde{u_{\imath} u_{J}}-\tilde{u}_{i} \tilde{u}_{j}
$$

This subgrid stress needs to be modelled by a SGS model so that the governing equations can be solved. A typical SGS model is the Smagorinsky model which can be defined as,

$$
\tau_{i j}=-2 v_{e} \bar{S}_{i j}
$$

Where $\bar{S}_{i j}$ is the filtered strain rate tensor and $v_{e}$ is the eddy viscosity coefficient. The strain rate tensor and eddy viscosity coefficient can be further described as,

$$
\begin{gathered}
v_{e}=\left(K_{s} \Delta\right)^{2}\left(2 \bar{S}_{i j} \bar{S}_{i j}\right)^{1 / 2} \\
\bar{S}_{i j}=\frac{1}{2}\left(\frac{\partial \tilde{u}_{i}}{\partial x_{j}}+\frac{\partial \tilde{u}_{j}}{\partial x_{i}}\right)
\end{gathered}
$$


Where $\Delta$ is the characteristic length scale and $\mathrm{K}_{s}$ is the Smagorinsky constant. The Smagorinsky constant can be selected based on the type of flow. For example, a value of 0.18 can be used for isotropic turbulence and flows near a solid wall can use a value of $0.1[83]$.

It should be noted that for this Smagorinsky model, eddy viscosity will be nonzero when a velocity gradient exists. At solid boundaries this will produce a nonzero value while in fact it should be zero since there is no turbulence. As such, additional constraints are needed for LES near-wall modeling. Typically, a Van Driest damping function is added into the length scale $(\Delta)$ in Equation (8.4) to correct this. This damping function can be described by,

$$
D=1-\exp \left(-y^{+} / A^{+}\right)
$$

Where $\mathrm{y}^{+}$is a non-dimensional wall distance for wall bounded flows and $\mathrm{A}^{+}$is the Van Driest damping constant (typically a value of 26 ).

Despite the subgrid resolution of LES, this model can still encounter difficulties in describing the near wall flow structures. Close to the walls the flow becomes dominated by vortices with characteristics lengths much smaller than those in the free flow. As such, in order to resolve the subgrid scale, mesh resolutions have to be increasingly fine close to the wall which makes a wall resolved LES simulation very expensive to perform.

To overcome these inherent issues with LES, hybrid RANS/LES techniques have been developed. These models divide the computational domain into subdomains 
where some of them are treated with RANS methods and others are treated with LES methods. Where RANS methods apply, the SGS model is replaced by a new turbulence model which can be defined as a combination of a RANS and SGS model. This allows for the development of a robust subgrid model $[56,83]$. An example of this hybrid RANS/LES technique is the detached-eddy simulation (DES). This model uses RANS methods in near-wall regions but incorporates LES methods for the rest of the flow. 